\title{
Manifestation of Pathological States of Numerous Diseases in the Largest Organ of the Human Body: (II) From Pancreatitis to Pancreatic Cancer Invasion, Formation of Stroma around the Primary Tumor in the Fascia, to Early Detection of Non-Coding microRNAs in Body Fluids and Development of Drugs to Treat Different Stages of Pancreatic Cancer
}

\author{
Peter Chin Wan Fung1* ${ }^{*}$ Regina Kit Chee Kong² \\ ${ }^{1}$ Division of Medical Physics, Department of Medicine, University of Hong Kong, Hong Kong, China \\ ${ }^{2}$ School of Traditional Chinese Medicine, Southern Medical University, Guangzhou, China \\ Email: *peterallegro333@gmail.com
}

How to cite this paper: Fung, P.C.W. and Kong, R.K.C. (2020) Manifestation of Pathological States of Numerous Diseases in the Largest Organ of the Human Body: (II) From Pancreatitis to Pancreatic Cancer Invasion, Formation of Stroma around the Primary Tumor in the Fascia, to Early Detection of Non-Coding microRNAs in Body Fluids and Development of Drugs to Treat Different Stages of Pancreatic Cancer. International Journal of Clinical Medicine, 11, 618-718.

https://doi.org/10.4236/ijcm.2020.1110052

Received: September 29, 2020

Accepted: October 28, 2020

Published: October 31, 2020

\begin{abstract}
Patients suffering from pancreatic ductal adenocarcinoma (PDAC) have an average survival time of $4-6$ months after confirmed diagnosis. The primary tumor is surrounded by a thick interstitial fluid with high pressure and dense distribution of collagen, forming a huge stroma, rendering the tumor resistant to chemo- and radiotherapy. From the genetic point of view, pancreatic carcinogenesis is driven by mutations, resulting in common activation of the oncogene KRAS, and/or inactivation of one or more of the tumor suppressor genes CDKN2A, TP53, SMAD4 [1]. The pancreas is a mixed exocrine and autocrine organ, with different cell types building up the organ. The pathogenesis involves more than 13 signaling pathways at different stages. Off-balance of the function of the proteins in these pathways due to the stated 4 plus other mutations could readily lead to carcinogenesis. We first present the basic mechanism of these 13 relevant pathways. We then provide a detailed analysis of the progression of this disease, from pancreatitis to tumor formation and metastasis, with special attention on the roles played by the newly dis-
\end{abstract}


Copyright $\odot 2020$ by author(s) and Scientific Research Publishing Inc. This work is licensed under the Creative Commons Attribution-NonCommercial International License (CC BY-NC 4.0). http://creativecommons.org/licenses/by-nc/4.0/ (c) (i) (8) Open Access cover calcium channel Piezo, stellate cells, stem-cell-like cells, and the concept invadopodium. Thirty potential drugs, based on in vitro and xenograft experiments from different groups, are discussed, including vitamins A, Tocotrienols-E, and D, chemical compounds, non-coding micro RNAs, circular RNA, piwi-interacting RNAs. The recent detection of exosomes enclosing many of these RNAs in body fluids gives us hope of developing early detection methodology because these RNAs carry messages for cell-cell communication at a distance. Delivery of potent drugs by nanoparticles gives us chance to send drugs through the stroma to target the tumor. Since body fluids form a circulating system, together with the connective tissues (where the tumor is associated) form the largest organ-the fascia, we conclude that manifestation of successive pathological states of pancreatic carcinogenesis can be found in compartments of the fascia. We present 17 figures, hoping to ease off the complexity of the pathogenesis of this most lethal cancer disease.

\section{Keywords}

Pancreatic Ductal Adenocarcinoma, Signaling Pathways in Carcinogenesis, Piezo 1,2, Exosomes Containing Micro RNA in Body Fluids, Stellate Cell, Cancer Stem Cell, Potential Agents to Treat Pancreatic Cancers, Fascia

\section{Introduction}

\subsection{Interaction of the Fascia and the Organs}

During embryonic development, wound healing and tissue homeostasis in adulthood, there is a complicated cellular network linking different biochemical, biophysical cellular pathways that result in 1) proliferation, 2) differentiation, 3) migration, 4) cell cycle progression, 5) apoptosis, and 6) autophagy. In general, groups of different cell types build up an organ (which can be a small blood vessel or a small nerve fiber). All organs are embedded by the largest organ of the body-the fascia, which is an intriguing interwoven structure of collagen fibrils/fibers, proteoglycans, glycoproteins, interstitial fluid, with resident cells and cells in transit. Different parts of the fascia have a special arrangement of their constituents to cater to the functions of organs embedded by these parts of the fascia (for details, see [2]). In the close neighborhood of an organ, the part of the fascia is generally called extracellular matrix (ECM). Fibroblasts apply tract forces to collagen fibers (which contain also elastic fibers) and transmit the force to other cells and connective tissues in the fascia. Such tension can change the phenotyping of the fibroblasts. Mast cells send out biochemical signals for physiological and immunity processes. Mesenchymal stem cells can change phenotypes according to the biophysical and biochemical signals received. The numerous compartments of the fascia, i.e. the ECMs (with the resident cells and cells in transit) associated with different organs (which can be a blood vessel, a nerve fiber) are reactive to the associated organs by mechanotransduction. 
Inside the fascia, which contains the interstitial fluid as a ground substance, there are growth factors such as the transforming growth factor molecules, that are stored in a dormant state. Mechanical force can release these growth factors that are (naturally) ligands for special receptors at the cell membrane. Once docked at the receptor, one or more cellular processes leading to events stated in 1) - 6) can be initiated. Cells in the fascia, or cells in the bloodstream, can generate cytokines, hormones that can pass from the blood vessel by extravasation to the interstitial fluid and these secretions can also be received by the specific receptors at the cell membrane of an organ(s). Moreover, there are special channels to allow passage of these biochemical molecules and others (such as ions in the extracellular stores) into the cells. In a healthy organ, biophysical and biochemical interactions between the ECMs and the organs would intriguingly trigger off cellular processes 1) - 6) in a balanced manner, so that homeostasis of the physiological processes are maintained with no manifestation of pathological states of the organs, for survival.

However, mutations of genes often happen, leading to the existence of defective proteins/peptides in the cytoplasm. If these defective proteins are not corrected (by rewinding) by heat shock proteins, or sent to apoptosis inside the cell, with lysosomes playing important roles (see details in [3]), these defective proteins exist in the cytosol of the cell. If these defective proteins happen to be members participating in one of the 1) - 6) pathways, cytokine-induced inflammation, abnormal growth, early apoptosis, uncontrolled cell-cycle progression, improper differentiation occur. These pathological states could lead directly or indirectly to carcinogenesis at the end. We have previously explained in detail the manifestation of the pathological states of the tendons diseases in the fascia [2]. We will show in this paper signatures of carcinogenesis of the most lethal cancer can be found in the fascia. In fact, detection of these signatures in the fluids of the fascia and drug delivery via the interstitial fluid gives us hope for early detection and therapeutic treatments.

\subsection{Objectives and Setting of This Article}

There are different cells in a pancreas and pancreatic cancer of any cell type, often resulting in pancreatic ductal adenocarcinoma (PDAC), which is the 4 th leading cause of deaths in the USA and is well known, unfortunately, to have the worst prognosis among all cancers. The mean survival period of PDAC is only 4 - 6 months [4]. It is estimated that PDAC (the disease) would become the second leading cause of cancer-related deaths in the USA before 2030 [5].

Compared to other cancers, PDAC is not a heavily mutated tumor. Recently, only four genes have been identified to be associated with the highest frequency of occurrence involved in the carcinogenesis of PDAC in humans: 1) KRAS (Kirsten rat sarcoma viral oncogene homolog), 2) CDKN2A (cyclin-dependent kinase inhibitor 2A, or p16), 3) TP53 (tumor protein 53) and 4) SMAD4 (mothers against decapentaplegic homlog-4). Other mutations have been found in dif- 
ferent pathways during carcinogenesis also in the PDAC specimens [6]. The objectives of this article are as follows: a) Review and extend the signaling pathways involved in different stages of pathogenesis of pancreatic cancers. There are more than 10 such signaling pathways (Sections (3)-(11)), the content of which is certainly relevant to the later part of the paper. At the end of each crucial pathway, at least one chemical as potential treatment (mainly compounds) is introduced, though most of them have not undergone clinical trials. b) Since the pancreas is a "mixed organ" with an autocrine and an exocrine function, different types of cells build up this organ. We analyze the progression of pathogenesis in Sections (12)-(17), starting from pancreatitis, through the epidermal to mesenchymal transition (EMT), finally to PDAC [7], leading to the presentation of an overall picture of tumorigenesis in Section (17). During our analysis, we pay special attention to the roles played by the stellate cell, the (pancreatic) stem cell-like cells, the newly discovered calcium ion channel Piezo1 [8], the concept of invadopodium [9], and the formation of the stroma [10]. c) Review the status of prevention of these diseases, plus other plausible treatments targeting proteins/genes in the pathways already discussed, including the functions of vitamins A, D, and tocotrienol-E; such analysis is included in Section (18). Section (19) is devoted to treatments with micro RNAs with efficacy based on in vitro evidence and in vivo animal xenografts investigation. The efficacies of some experimental results cannot be pinned down to specific pathways so far known. d) To look for early detection and therapies of this most lethal cancer, we describe specific examples of the non-coding circular and long non-coding RNAs in Section (20). These non-coding RNAs act as a sponge to "absorb" or interact with other RNAs and therefore have profound effects on the augmentation or attenuation of the pathogenesis. It has been discovered in the recent several years that these non-coding RNAs are messages conveying the status of the sending cell and being carried by vesicles. These vesicles have been found in several body fluids. The concluding remarks in Section (21) summarise the analysis of this paper with special attention to the fact that the pathological state of the progression of pancreatic tumorigenesis manifests itself in different compartments of the fascia [2], which includes the interstitial and other body fluids that carry those vesicles just mentioned. We also emphasize the fact that a huge stroma is developed around the primary tumor in the extracellular matrix, with a high density of collagen fibrils and viscous interstitial fluid, rendering the well-known chemotherapy and radiotherapy resistance. We see the hope of early detection of the vesicles in the fascia compartment just mentioned and consider the usage of nano-particles to deliver "drugs" as a workable strategy; these drugs could be pharmaceutical, micro RNA mimics (or their inhibitors). All the crucial statements of deductions are justified with the experimental results of different research groups. 17 figures are presented for the convenience of the reader to follow the complicated signaling pathways and deductions. A list of abbreviations for the relevant biological/biochemical names is provided at the end of the article. 


\section{Some Basics of the Pancreas-The Pancreas Is a Mixed Gland with Endocrine and Exocrine Functions}

\subsection{The Islets Containing $\alpha, \beta, \delta$, and $\gamma$ Cells That Secrete Different Hormones through the Blood Vessels to Fulfil Endocrine Functions}

As an endocrine gland, the pancreatic islets (painted in light green with a thin red boundary in Figure 1) of Langerhans secrete mainly four hormones: 1) insulin, 2) glucagon, 3) somatostatin, 4) pancreatic polypeptide, into the bloodstream. The enlarged diagram of an islet is shown in the lower right corner of Figure 1. Each islet is surrounded by about $20 \%$ of (total cells in each islet) $\alpha$ cells (red), which secrete glucagon, a hormone that prevents blood glucose dropping to too low a level. There are blood vessels in each islet, bringing glucagon to the liver where some stored glycogen is converted to glucose. $70 \%$ of the cells in each islet are $\beta$ cells (light green), which secret insulin, that is delivered first by the blood, and then by the interstitial fluid to the muscles. The third type of cells in each islet are the $\delta$ cells (yellow, several percent of cells in an islet) which secret somatostatin, a hormone that inhibits the secretion of glucagon and insulin as a regulator of these two hormones. Stimulated by eating, exercising, and fasting, the $\gamma$ cells secrete pancreatic polypeptide, which can inhibit gallbladder contraction and pancreatic exocrine secretion; the mechanism of secretion and function is still unclear [11].

\subsection{The Spleen as a Blood Filter and Blood Cells Storage Provides Clean Blood Supply and Immunity Service to the Pancreas}

Blood vessels are connecting the pancreas and the spleen, whose main functions are: 1) As a filter for blood-old red blood cells are recycled in the spleen. 2) Storage of platelets and white blood cells. The spleen is an important organ of the immune system.

The pancreas is thus a main part of the digestive system and source of "fuel" (in the form of hormones stated above) for skeletal movement and organ function in general. The malfunction of the pancreas would lead to defects in the functions 1) and 2) of the spleen too [12].

\subsection{As an Exocrine Organ, the Acinar Cells with Their Zymogen Digestive Enzymes Secrete Digestive Juice into an Epithelium (the Duodenum, Rather Than the Bloodstream) through the Pancreatic Duct; the Pancreas Also Provides a Route for the Bile into the Duodenum from the Gallbladder (as Storage) and the Liver}

As a digestive organ, it secretes 2) pancreatic juice that contains bicarbonates for the neutralization of stomach acid that enters the duodenum region as well as digestive enzymes (Figure 1) that break down fats, proteins, and carbohydrates of the food that has entered the duodenum region. Moreover, the bile duct of the gallbladder, passes through the duodenal pillar near the "head" of the pancreas, 
supplying bile to digest fat too. The main type of pancreatic cells, the acinar cells (purple) form ring-shaped structure attached to the pancreatic duct (light purple) via the square-shaped centroacinar cells (light blue) and (branch) ductal cells. The acinar cells synthesize Zymogen granule digestive enzyme, which in turn secretes the pancreatic digestive juice into the duodenum via a branch of the pancreatic duct. The bile duct (deep green) from the gallbladder has a zig-zag structure, and together with the hepatic duct (also deep green), forms a single duct joining the pancreatic duct at the head of the pancreas. This "mixed duct" pierces through the connective tissues of the pancreas and the duodenum to supply bile juice plus digestive juice to digest food-the entrance to the duodenum is shown by the white arrow. In some people, this joint tube is separated into two (see X-ray photo in Figure 225.1 of [13]).

The adult pancreas is highly plastic to keep its integrity in response to internal stress such as activation of digestive enzymes, obstruction of pancreatic ducts due to the development of gallstones. It can also suffer from external stresses such as trauma and alcohol interaction. Since this organ is rather fragile to mechanical injury, it is anatomically protected by the diaphragm during evolution. Only the major cells are labeled in Figure 1, without the immunity cells repairing this organ after injury. During pancreatitis, acinar cells undergo a morphologic and transcriptional change into duct-like cells with embryonic progenitor

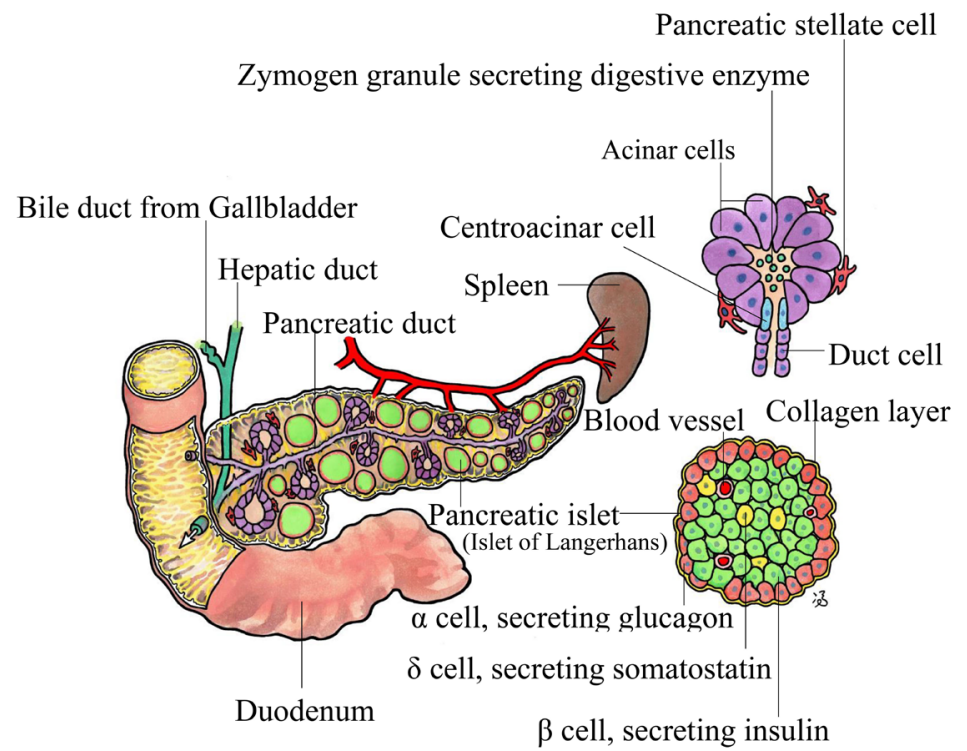

Figure 1. The pancreas, duodenum, pancreatic duct, acinar cells (enlarged in the upper right corner), islets (enlarged in the lower right corner). The deep green ducts deliver bile juice from the gallbladder (as storage) and liver and merge with the pancreatic duct in most people. Digestive juice plus bile juice are secreted into the duodenum, whereas several hormones (glucagon, insulin, somatostatin) are secreted by the islet cells into the blood vessels. An islet is composed mainly of $\alpha$ cells (red), $\beta$ cells (light green), and $\delta$ cells (yellow). The pancreatic stellate cells are painted in red-they play active roles in cancer formation and metastasis. There are some stellate cells near the islets too (not shown here) See text for more details. This figure was painted by author PCWF. 
cell properties, developing back to adult acinar cells at the end stage of the repairing process; such a wound healing process is now called acinar-to-ductal metaplasia (ADM) [14]. Note that the exocrine part of the pancreas composes at least $95 \%$ of the organ's mass.

\subsection{The Stellate Cells Plus the Stem Cell-Like Cells Are Found around the Acinar Cells and Quiescent Stellate Cells Are Found in the Islets of Rat Recently}

The red cells around the enlarged "acinar ring" in the upper right corner are pancreatic stellate cells, plus some stem-like cells, which will be shown later to play important roles in pancreatic carcinogenesis and metastasis, if activated into the myofibroblast form [15]. Recently quiescent stellate cells have been found in the islets of rat; we infer that such distribution of cells is also similar in human islets [16].

\section{The Several Conserved Basic Pathways in Most Cells}

\subsection{The Akt-mTOR 1,2 and LKP1-AMPK-(TSC1/TSC2) Pathways in Almost All Cell Types-The Imbalance of Any One or More Would Lead to Disease States, Including Those to Be Discussed Later in This Article}

Let us first turn to the upper right side of Figure 2. Receptor tyrosine kinases (RTKs, painted in red) are cell surface receptors for many (polypeptide) growth factors, cytokines, and hormones, including insulin. Phosphatidylinositol-3 kinase (PI3K, yellow), which is a signaling molecule, is recruited to the cell membrane by the activated growth factor receptors. Phosphatidylinositol 4,5-bisphosphate or $\mathrm{PIP}_{2}$ (yellow) or called $\mathrm{PI}(4,5) \mathrm{P}_{2}$, is a small phospholipid component of the cell membrane. Being activated by PI3K, the lipid unit $\mathrm{PIP}_{2}$ converts to another lipid unit $\mathrm{PIP}_{3}$ (yellow, also residing inside the cell membrane), leading to the activation of the phosphoinositide-dependent protein kinase-1 (PDK1, yellow), which is also called the "master kinase PDK1".

Now PDK1, as the "master kinase", activates the following enzymes: 1) Protein kinase A, (PKA) whose activity is dependent on cellular levels of cyclic AMP and is a regulator of glycogen, sugar, and lipid metabolism. 2) Protein kinase $\mathrm{C}$, (PKC), a member of the family of protein kinase enzymes that are involved in controlling the function of other proteins. 3) Ribosomal protein kinase $\beta-1$ P70-S6K (or S6K1), the phosphorylation of which induces protein synthesis at the ribosome (already mentioned in the synthesis process of proteins). 4) Protein Kinase B or Akt (yellow). In the figure, mTOR1,2 proteins stand for mammalian target of rapamycin complex 1,2. Now Akt activates the mTOR2 protein (activation is a two-way process [17].

The PI3K/Akt/mTOR1,2 pathways play key roles in numerous normal cellular functions including proliferation, cell adhesion, metabolism, migration, invasion (during growth and wound repair), and survival [18]. During the past three decades, many of the up-stream and down-stream regulations have been discov- 
ered. Here we emphasize only the functions of the relevant enzymes involved in proliferation, angiogenesis, apoptosis, migration (including invasion).

In the "growth state", a GTP-binding protein Rheb (also known as Ras homolog enriched in the brain, painted in green) causes mTOR1 to trigger gene expressions in the nucleus, and protein biosynthesis. A ribosome, which is generally free in the cytosol, is needed for such synthesis. A ribosomal protein kinase $\beta$-1 P70-S6K (or S6K1, yellow) helps to activate the ribosome, whose small unit is then attached to mTOR1 and the large unit is attached to the rough part of an endoplasmic reticulum (ER). One ribosome (bright green) structure is schematically shown in the figure, being attached to mTOR1. When a gene is triggered off to synthesize the related protein, the RNA "zipped" through between the small and big units of the ribosome; with the help of transmission RNA (tRNA, light yellow), amino acids are picked up and a related native protein is synthesized. This native protein is fed into the ER for quality control: heat shock proteins (such as HSP 70 and HSP 90) check and refold the misfolded native or "first round proteins". Hence the function of HSPs is necessary for a healthy cell to synthesize proper proteins. In the lower-left side of the figure, mTOR1 also activates heat shock factor 1 (HSF1) which leads to the synthesis of HSPs in the nucleus, as indicated by the arrow and the patch marked "HSPs expression".

The growth state must be regulated by the "AMPK pathway" as a negative control. The liver kinase B1 protein (LKB1, green) functions as a tumor suppressor. It activates the 5' adenosine monophosphate-activated protein kinase (AMPK, green) which in turn mobilizes the tuberous sclerosis complex (TSC), that is built of two basic units: TSC1 (also called hamartin) and TSC2 (also called tuberin). This complex stops the action of Rheb, and thus stops protein synthesis.

Note that mTORC2 (orange) is located close to a mitochondrion (purple and white) and an endoplasmic reticulum (deep green). The mitochondrion generates energy $(\varepsilon)$. The bioenergy $\varepsilon$ in the cytosol inhibits the action of AMPK (back to the left side of the figure), giving the chance of activation of mTOR1 and growth. Moreover, the protein FOXO1,3 leads to apoptosis, as protection of the host, during the condition of insufficient bioenergy or pathological state [19]. Hence, sufficient intracellular energy also rescues the cell from apoptosis. Besides, there is also evidence that Akt can inhibit the action of the (TSC1/TSC2) complex [20], letting the protein Rheb free to mobilize mTOR1 for growth. The regulation of the PDK1-Akt pathway is based on the inhibition of the phosphatase and tensin homolog protein (PTEN) on the conversion of $\mathrm{PIP}_{2}$ to $\mathrm{PIP}_{3}$, thus stopping the action of the PDK1-Akt pathway altogether [21]. The processes described above are balanced intriguingly, for most cell types. Now we understand that the duty of PTEN is very important, and mutation of the gene encoding this protein would readily lead to carcinogenesis. The other two pathways on the extreme left and right will be discussed in the following section. 


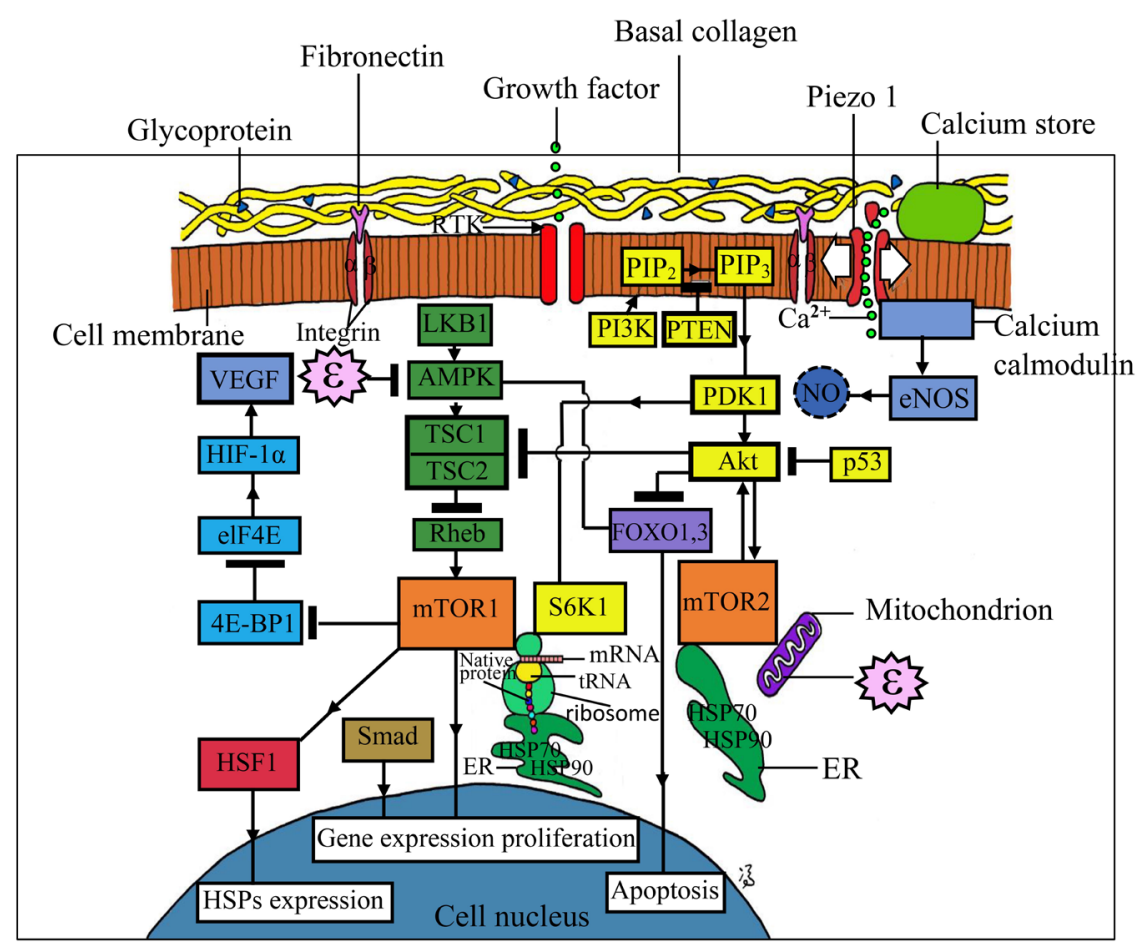

Figure 2. The LKP1-AMPK-(TSC1/TSC2) pathway (dull green) inhibits cell proliferation and protein synthesis. The PI3K-PIP2-PIP3-PDK1-Akt pathway (yellow) stimulates cell growth. Protein Rheb triggers cell proliferation also. Protein PTEN is a key tumor suppressor. HSF1 activation leads to the synthesis of various heat shock proteins (HSPs) for quality control of synthesized proteins. The two blue pathways on the extreme left and right pertain to the endogenous synthesis of vascular endothelial growth factor (VEGF) and nitric oxide (NO) for vessel dilation, the details of which will be amplified in the next section. For other details, see text. This figure was hand-painted by author PCWF.

\subsection{Synthesis of Vascular Endothelial Growth Factor (VEGF) and Nitric Oxide-These Two Processes Are Physiological during Embryogenesis and Wound Healing, But Are Involved in Basically All Types of Carcinogenesis}

The eukaryotic translation initiation factor $4 \mathrm{E}$ (eIF4E)-binding protein 1 (4E-BP1, encoded by the gene EIF4EBP1), is a member of a family of translation repressor proteins [22]. The protein 4E-BP1 directly interacts with eukaryotic translation initiation factor $4 \mathrm{E}$ (eIF4E), whose activity is then inhibited; these two proteins are commonly treated as a complex in specifying pathway activation. $4 \mathrm{E}-\mathrm{BP} 1$ is a well-established substrate of the rapamycin (mTOR1) signaling pathway [23]. Now, eIF4E activates the hypoxia-inducible factor-1a (HIF-1a), leading to a synthesis of the vascular endothelial growth factor (VEGF), resulting in angiogenesis. Note that HIF-1 $\alpha$ is a master transcriptional regulator of cellular response to hypoxia. In short, angiogenesis occurs when mTOR1 is in action (by growth factor stimulation, such as insulin, as one example) [24]. This pathway is shown on the left side of Figure 2. During embryonic development or wound repair, cells would have to synthesize VEGF for blood vessel growth.

Recently the calcium channels Piezo1,2 have been discovered in cell mem- 
branes of many cells, including cells of islets in the pancreas [25]. A Piezo1 channel (relevant to pancreatic cells) is schematically painted in Figure 2. At the quiescent state, the entrance is blocked. When triggered by mechanotransduction, such as that activated by integrins in the cell membrane, the calcium channel is opened to calcium ions from extracellular stores. Calcium ions mobilize a small protein called calcium calmodulin (blue), which is attached to the inner side of the cell membrane. The protein calmodulin activates the enzyme endothelial nitric oxide synthase (eNOS, one of the three isoforms, painted in blue) which converts $\mathrm{L}$-arginine to citrulline, releasing nitric oxide $(\mathrm{NO})$ that can pass through water and cell membrane, and can be delivered by red blood cells [26]. The NO generation process is schematically represented at the upper right corner of Figure 2. Cancer cells need a lot of oxygen and nutrients to be able to survive. To cancer cells, angiogenesis is the first establishment. Moreover, all blood vessels receive vessel dilator and constrictors to adjust the blood flow rate for the obvious reason of survival. NO is a strong vessel dilator (as against angiotensin). Hence cancer cells need to generate a large amount of NO molecules. With the excessive generation of VEGF and NO, the "angiogenic switch" of pancreatic cells could readily occur, as mentioned in [27].

\subsection{The Anti-Cancer Effects of Irisin (Hormone) Targeting the AMPK-mTOR1 Pathway}

Uncoupling protein 1 (UCP1) is a membrane protein found in the mitochondrial inner membrane of brown adipose cells. Activating UCP1 by cold or diet, energy derived from the oxidation of fat is not available to drive the phosphorylation of ATP and is consequently dissipated as thermal energy, playing a role in regulating energy metabolism. Irisin is an exercise-induced cytokine, produced by the proteolytic hydrolysis of fibronectin type III domain-containing protein 5 (FNDC5) [28]. There is also evidence that Irisin could induce browning of white adipose cells by upregulating the peroxisome proliferator-activated receptor (PPAR) $-\gamma$ and UCP1.

Recently, apart from the secretion from the muscle and skin, Irisin has been identified also as a myokine in the heart and organs of the digestive system, including the pancreas [29]. Using human pancreatic cancer cell lines MIA PaCa-2 and Panc03.27 (pancreatic adenocarcinoma epithelial cells), it has been shown that 1) Irisin receptors could exist on the surfaces of pancreatic cancer cells. 2) Irisin inhibits pancreatic cancer cell growth via the AMPK-mTOR1 pathway (see Figure 2).

In another study [30], employing the human pancreatic cancer cell lines, PANC-1 (isolated from a pancreatic carcinoma of ductal cell origin) and BxPC-3 (adenocarcinoma origin), it has been reported that 1) Irisin decreased the proliferation of pancreatic cancer PANC-1 and BxPC-3 cells. 2) Irisin's action led to apoptosis of the pancreatic cancer BxPC-3 cells. 3) Migration and invasion of pancreatic cancer BxPC-3 cells were attenuated by the application Irisin. 4) Irisin suppressed the protein phosphorylation of the PI3K/Akt signaling pathway. Re- 
ferring to Figure 2, suppression of this pathway amounts to inhibiting the action Akt to stop apoptosis via FOXO1,3, with the result of letting the cancer proceed to natural cell death. Both the studies of [29] and [30] substantiate each other using four different human pancreatic cancer cell lines.

Developing drugs that can promote the endogenous secretion of Irisin seems to be one plausible therapy to treat pancreatic cancer. We should note, however, this peptide has no and even adverse effects on a few other types of cancer, as remarked in [31]. The first chemical synthesis of irisin using two sequential ketoacid-hydroxylamine (KAHA) ligation giving milligram quantities has been reported four years ago [32].

\section{The Most Significant Pathway Responding to Mechanical Stimulation Initiated in the ECM and Its Correlation with Pancreatitis}

\subsection{The Mitogen-Activated Protein Kinase (MARK) Cascade or the Ras-Raf-Mek-Erk Signal Transduction Cascade Triggered by the Newly Discovered Calcium Ion Channel Piezo1 Plus Internal Calcium Store, Leads to Transcription, Mitosis}

The (activation) mutation of the KRAS gene has been found to be at $~ 95 \%$ in late stages of pancreatic cancer [1]. The gene KRAS encodes the protein Ras. We will discuss straight away the signaling pathway initiated by the over-activation of Ras here. Referring to Figure 3, there are many receptor tyrosine kinases (RTKs, reddish-orange) at the cell membrane; activation by growth factors (like epithelial growth factor, EGF) when docking on the receptor caused activation of the enzyme phospholipase enzyme (PLC-epsilon1, bluish-green) by these growth factors. PLC-epsilon1 is attached to the cell membrane and catalyzes the hydrolysis of phosphatidylinositol-4,5-bisphosphate $\left(\operatorname{PtdIns}(4,5) \mathrm{P}_{2}\right)$, or $\mathrm{PI}(4,5) \mathrm{P}_{2}$ or $\mathrm{PIP}_{2}$ (see Figure 2) bluish-green) to give two second messengers: 1) phosphatidylinositol 1,4,5-triphosphate ( $\mathrm{IP}_{3}$, also bluish-green) and 2) diacylglycerol (DAG, light purple, not shown in Figure 2) [33]. There is evidence this protein Ins $(1,4,5) \mathrm{P}_{3}$ docks the receptor (represented by the symbol $\mathrm{R}$, dull green) in the surface of endoplasmic reticulum (deep green). The internal calcium ion store (medium green) is then opened, releasing $\mathrm{Ca}^{2+}$ (bright green small circles) [34] to a concentration of even up to micro-Mole level, much greater than the value of 100 nano-Mole, which is the value during the rest state. So that is the source intracellular calcium ions.

Note that DAG activates the protein called Ras (light purple, encoded by the gene KRAS) which is a member of a class of proteins called small GTPase, and these proteins are expressed in all animal cell lineages and organs, involving mainly in transmitting signals within cells. DAG can activate Ras only if calcium ions are present, and Ras is then "switched on". Ras can also be switched off in the absence of $\mathrm{Ca}^{2+}$. The active Ras subsequently activates the Rapidly Accelerated Fibrosarcoma, or Raf protein (light purple). Raf causes the phosphorylation of Mitogen-activated protein kinase (Mek, light purple), extracellular sig- 
nal-regulated kinases (Erk, light purple) in a series. Activated Erk phosphorylates Ribosomal s6 family kinases (RSK, light purple). The Ras-Raf-Mek-Erk pathway is then said to be formed. Both RSK and Erk (after activation) translocate to the cell nucleus (deep blue) where they activate multiple transcription factors resulting in effector protein synthesis; there are consequential causal changes in cell growth, differentiation, and survival-we use only the terms transcriptions and mitosis to represent such consequences in Figure 3 [35]. The above Ras-Raf-Mek-Erk pathway is also called mitogen-activated protein kinase (MARK) cascade. Apart from the fat cell, this cascade functions in basically all other cells in the body.

By 2017 , more than $90 \%$ of pancreatic cancers have been found to have mutations in the form of amplifications, deletions, translocations, inversions, frameshifts, and substitutions [1]. Aberrant KRAS mutation, resulting in aberrant RasRaf-Mek-Erk signaling, activates inflammatory signaling pathways that play crucial roles in promoting pancreatic intraepithelial neoplasia (PanIN) and the progression of PDAC [36].

At the molecular level, genes mutation can first enhance/subside the function of a related protein, making it even constitutively active or loss of function, with complete abrogation in the extreme case. Subsequently, the changes in protein functions can cause uncontrolled proliferation, motility, and adhesion of cells, protection from apoptosis or autophagy, DNA repair problems, the establishment of a cancerous niche, resulting in the development and growth of cancers. The Ras protein is a small GTPase, which binds guanosine triphosphate and diphosphate nucleotides. It is activated or deactivated when bound to GTP or GDP respectively. At the protein level, activated protein Ras binds and activates Raf family kinases, Raf1. Activated Rafs phosphorylate and activate Mek1,2 kinases, which in turn phosphorylate and activate Erk1,2. The Ras-Raf-Mek-Erk pathway eventually leads to transcription, cell proliferation, and mitosis as explained briefly above.

In passing, note that the protein PI3K (orange), which is attached to the cell membrane, is drawn in both Figure 2 and Figure 3. The activation of the PI3K-PDK1-Akt-mTOR1,2 has already been discussed in the last section. The crucial consequence of that pathway is the blockage of the apoptotic process, rendering this cell viable. The same protein PI3K appears in both figures. We show a short symbol "inhibits apoptosis" in Figure 3 to highlight this fact, referring the readers to Figure 2 above again.

The two main pathways shown in Figure 3 are: 1) the Ras-Raf-Mek-Erk pathway and 2) the Rho-Rock pathway. Note that integrin can be activated by mechanical force from ECM, and growth factors. The focal adhesion, composed of several proteins, is formed. Fibronectin has an affinity to integrin. Pathway 1) leads to transcription and mitosis, whereas pathway 2) initiates the formation of the F-actin (a-SMA) chain(s) starting from the focal adhesion. The "glued" $\alpha$-SMA chains can contract, leading to the morphological change of cell shape and migration. Some of these chains also join the nucleus surface, mediating 
transcription. Mechanical stimulation such as acupuncture/acupressure/massage causes grouping of the integrin sets together; the tension forces are extended on the cell membrane to pull the cation channel Piezo, leading to the entry of calcium ions from the external calcium store. The E-cadherin is a transmembrane protein joining adjacent cells (usually of the same type). The indices 1,2 in Piezo1,2 are omitted, as each type is found in specific cells. In a neuron, Piezo2 is found. In an epithelial cell, the channel is Piezol.

We still have not answered the question: where do the $\mathrm{Ca}^{2+}$ ions come from to replenish the metabolized internal calcium ions? The recent discovery of the calcium channel Piezo1,2 provides the source of calcium entry from extracellular calcium store due to mechanical traction of the integrins in the cell membrane. This issue is followed up in the next section.

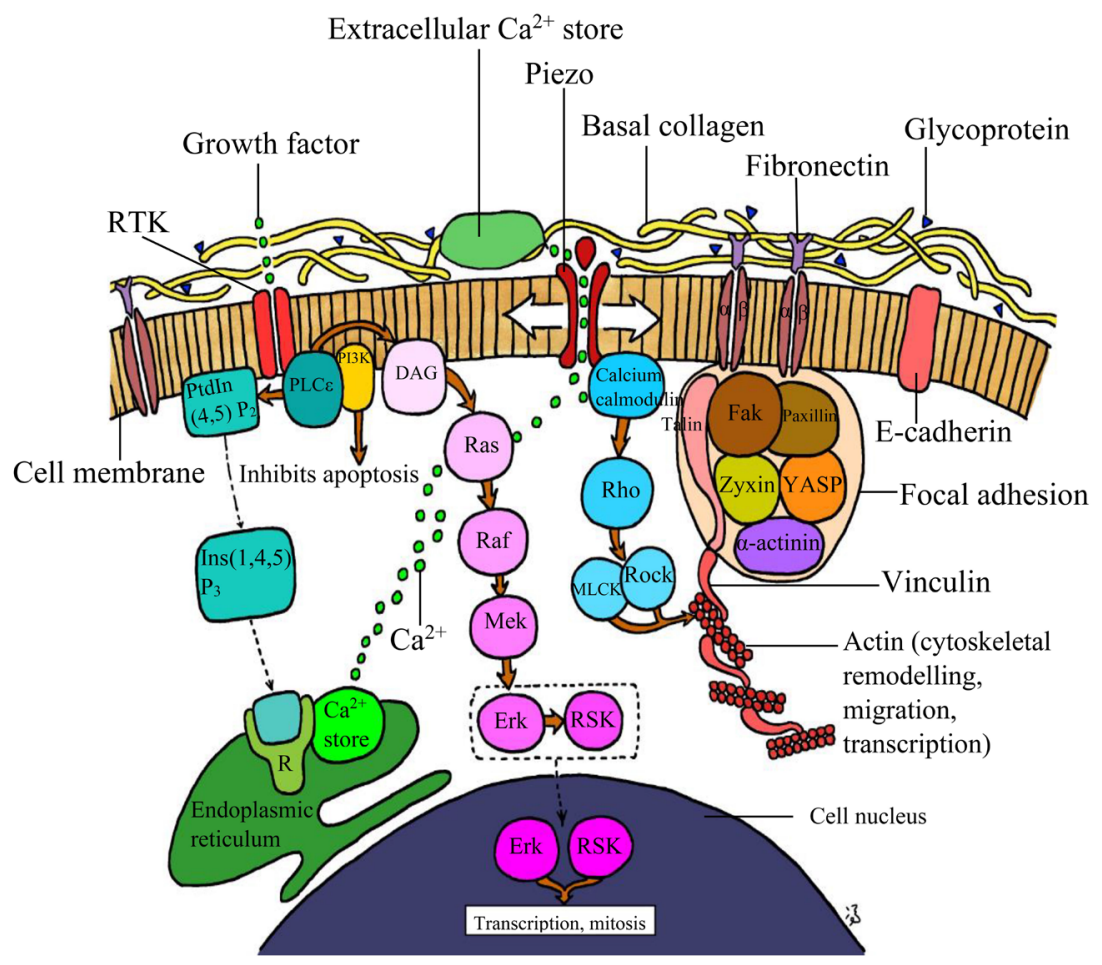

Figure 3. Emphasizing the several pathways in most cells resulting from mechanical stimuli applied at the extra-cellular matrix. The symbols inside the circular/elliptical "bags" are names of the proteins which are defined and specified in the text. The thin yellow fibrils are constituents of the basal membrane (composed of mainly collagen type IV) structure that embeds every organ. The small triangles in the ECM are proteoglycans/glycoproteins, which form part of the non-collagen proteins. RTK is a typical cell membrane channel that allows the docking of growth factors that activate Proteins PLC-epsilon and PI3K. The dash-dot line with an arrow means "becomes after phosphorylation or other chemical reaction". The deep orange arrows indicate the activation of proteins. We observe that on activation, both the internal and external calcium ion stores can release calcium ions which triggers (a) the Ras-Raf-Mek-Erk pathway and (b) the Rho-Rock pathway. Integrin can be activated by mechanical force from ECM, and growth factors. The focal adhesion contains several proteins as specified in Section (4.2), Piezo is a newly discovered calcium ion channel discussed in Sections (4.2) - (4.4). For other details, see text in Section (4.1). This figure was hand-painted by author PCWF. 


\subsection{The Important Role Played by the Newly Discovered Calcium Channel Piezo 1,2}

Referring to Figure 3, an integrin set has two units-the alpha (red) and the (slightly longer) Beta (red), the general function has been reviewed previously. When an integrin set is attached to the ligand (fibronectin, purple [37]); it becomes active, and the integrin set can recruit specific proteins forming a group called focal adhesion. According to function, the focal adhesion complex may be roughly divided into three layers. The signaling layer, closest to the integrins cell membrane, contains the highly phosphorylated signaling proteins 1) focal adhesion kinase (Fak, brown in Figure 3), which can be considered as a crucial integrator of cell mobility, and 2) Paxillin (lighter brown), whose main function is to adhere the cell to the ECM. Paxillin is expressed at focal adhesions of non-striated cells and at costameres of striated muscle cells. The second layer of the focal adhesion complex may be called the force transduction layer, which has members such as 3) Talin (pinkish) and 4) Vinculin (reddish-orange); they are adapter proteins that connect the integrin complex to the actin chain that can contract. Talin has an elongated structure, spanning basically the whole (force transduction) layer, with its head binding to integrins and the rod connecting actin (which can be $30 \mathrm{~nm}$ away from the integrins). Mechanical stretching of Talin promotes Vinculin binding; such binding stabilizes the focal adhesion complex [38]. The third layer may be called the actin regulatory and actin stress fiber layer. This layer contains proteins such as 5) Zyxin (ochre color), a zinc-binding phosphoprotein, serving as a messenger in the signal transduction pathway, by modulating the cytoskeletal organization of actin bundles. 6) Vasodilator-stimulated phosphoprotein (VASP, orange) and 7) Alpha-actinin (red), which is a microfilament protein. The boundary of this layer can be $60 \mathrm{~nm}$ away from the integrin layer. We have painted a boundary (peach color) surrounding the key proteins in the focal adhesion complex (there are other proteins too). Now, this focal adhesion complex is attached to one or more integrin sets. The formation of the focal adhesion is an important step in physiological functions and pathological progressions. We will follow up on what focal adhesion leads to, in relation to the action of the calcium ion channel, in the next section. In the meantime, we turn to the pathway involving one of the four most frequent mutated genes in pancreatic cancer.

When a group of integrin sets are activated, they are crowded together, leading to contraction of the cell membrane (light brown). At the quiescent state, there is a triangular structure like a cork of a wine bottle (built of amino acids) blocking the opening in a typical Piezo structure. We shall leave out 1 or 2 in the term Piezo1,2 as they are cell-type-dependent. When the integrin sets are in action, a transduction force (represented by the white arrows) automatically acts on the cation channels Piezo. Upon being pulled on both sides via mechanical stimulation, there is some space effectively opened to the entry of positive ions $\mathrm{Ca}^{2+}$, as governed by the arrangement of the residues (with electric 
polarization) as described in [8]. With such understanding, the internal calcium ions which are used in triggering the Ras-Raf-Mek-Erk signal transduction cascade are then replenished, a missing link confirmed only by 2010 [39]. With the rat model, using fluorescence imaging microscopy detection of dendritic spine of neuron in real-time, it has been demonstrated that the structural plasticity of the dendritic spine of neuron of rat first activates the small calcium calmodulin protein (near the cell membrane) and then the protein GTPase Rho A (see Figure 3). We propose that the structural plasticity would open the cation channel Piezo also. This proposal has been supported by recent experiments on several types of cells (see e.g. [8]). In turn, GTPase Rho A activates the Rho-associated protein kinase (Rock) plus Myosin light-chain kinase (MLCK). This Rock-MLCK complex activates myosin II protein which lines up the actin molecules, forming the actin chain(s), as shown in Figure 3 (see also [40]). When the actin chains are formed, cytoskeletal remodeling occurs, leading to migration. This actin structure can be extended to the nucleus, causing certain transcription of proteins depending on the mechanical stimulation sensed by the integrin-Piezo complex. Thus, the tension of the collagen fibrils/fibers outside the basal membrane, which embraces the cell groups of an organ (even a small blood vessel, a small nerve fiber) has profound effects on the proteins synthesized by the associated group of cells.

\subsection{Mechanical Pressure Applied to the Pancreas Would Readily Induce Acute Pancreatitis and the Evidence of Abundant Expression of Piezo1 in Acinar Cells of the Rat}

Recently, using the in vivo rat model, it has been demonstrated that channel Piezol is found to be expressed in pancreatic acinar cells based on RT-PCR analysis, plus the following observations: 1) Intraductal pressure would cause acute pancreatitis (using high-pressure transducer, as a starting point of the known phenomenon). 2) The acinar cells were full of calcium ions when stimulated (via calcium indicator Calcium 6-QF analysis). 3) Yoda1, which is a Piezo1 agonist, would induce acute pancreatitis. 4) GsMTx4 (inferred to be working as Piezol antagonist) would reduce the severity of acute pancreatitis 5) The degree of severity of pancreatitis in acinar cells with genetic deletion of Piezo1 is much less, using the rat model [8].

\subsection{Piezo1 Are Abundant in Pancreatic Stellate Cells of Mice Models and Also in Beta Cell Lines of the Rat's Islet}

Pancreatic ductal adenocarcinoma (PDAC) has a fibrotic stroma, known to be acidic. The stroma of the pancreatic ductal adenocarcinoma has a gel-fluid phase full of interstitial fluid, compressing the tumor with a high hydrostatic pressure up to even $100 \mathrm{~mm} \mathrm{Hg}$ by direct measurement using a transducer in mice model study [41]. Moreover, whereas the normal pancreatic juice is alkaline (to balance the stomach acid), the desmoplastic PDAC microenvironment is found to be acidic with a pH value below 6.5 [42]. High expressions of Piezo1 mRNA have 
been found in murine PSCs [43]. Stimulation of the Piezol channel led to calcium influx. In the in vitro experiment, PSCs were seeded on a matrix resembling the desmoplastic matrix found in the PDAC stroma; application of Piezol activator Yoda1 mobilized PSCs to migrate in such matrix (see Figure 3A of [10]).

There is a puzzle on the discovery of low $\mathrm{Ph}$ value in the tumor region because PSCs would have proceeded to apoptosis under such acidity. In animal studies, PSCs were found to invade together with the pancreatic cancer cells [10]. Piezo1 channels have been found in the beta cells (in the islets) of the rat's cell lines INS-1 and BRIN-BD11, and the ion channels participate in the insulin secretion mechanism [25].

\subsection{On the Efficacy of Gemcitabine Plus Nimotuzumab to Treat PDAC; the Evidence of Phenols and Sulfonamides Having Affinity to Mutated Ras Protein}

Aberrant Ras-Raf-Mek signaling plays a role in activating mutations found in pancreatic cancers of different stages/types (70\% - 90\%) [44]. The Kras (G12D) is a specific variation in the Ras; this variant, with no mutation at amino acid position 12, has a glycine. The mutated Kras G12D protein has an aspartic acid, or $\mathrm{D}$ for short in position 12 instead. In order to identify compounds that bind directly to this muted protein, a fragment screen [6] using uniformly ${ }^{15} \mathrm{~N}$ labeled GDP-bound Kras (G12D). From the NMR-based screen of 11,000 fragments, the approximately 140 fragments yielded have been identified to bind to GDP-bound Kras (G12D) in the study of [45]. Six small molecules were identified to have an affinity to mutated Ras protein: 1) phenols; 2) sulfonamides; 3) three analogs of 1) and 2) were synthesized (see Figure 1 of [45]).

A randomized study (with 192 patients) was carried out to investigate the efficacy of gemcitabine (gem) plus nimotuzumab (nimo), which is an anti-epidermal growth factor receptor monoclonal antibody, compared with gem plus placebo as first-line therapy in patients with advanced pancreatic cancer. Gemcitabine functions by blocking the creation of new DNA, resulting in cell death, and is a well-known nucleoside analog family of medication. One-year overall survival (OS)/progression-free survival (PFS) was 34\%/22\% for the combined gem plus nimo compared with $19 \% / 10 \%$ for gem plus placebo $(\mathrm{p}=0.02)$ [46].

\section{CDKN2A, Which Encodes an Essential Cell-Cycle Regulator, Is the Most Frequently Mutated (with Alteration) Tumor Suppressor Gene, with Loss of Function in More Than $\mathbf{9 0 \%}$ of Ductal Adenocarcinomas, Particularly in the Early Phases}

\subsection{The CDKN2A Pathway}

The gene CDKN2A has several splicing sites which can generate transcript va- 
riants. This gene, which encodes an essential cell-cycle regulator, is the most frequently mutated (with alteration) tumor suppressor gene, with loss of function in more than $90 \%$ of ductal adenocarcinomas [47] [48].

CDKN2A encodes the following two tumor suppressor proteins: 1) p16(INK4A) and 2) p14(ARF) proteins. The p16 (INK4A) protein binds to two other proteins CDK4 and CDK6 both of which normally stimulate the cell to continue through the cycle and divide. However, binding of p16 (INK4A) blocks CDK4's or CDK6's ability to stimulate cell cycle progression-p16(INK4A) is thus a tumor suppressor. However, as is noted in [49], long-term expression of p16 (INK4A) pushes cells to enter senescence. The p14(ARF) protein protects the well-known tumor suppressor protein $\mathrm{p} 53$ from being broken down; p14(ARF) is also a tumor suppressor. Overexpression of proteins 1) and 2) or the gene CDKN2A have anti-cancer effects.

In the cytosol, p14(ARF) protein inhibits the function of the human double minute $2(\mathrm{Hdm} 2)$ which forms a complex with another protein called Hdmx. This complex inhibits the action of p53, which activates a cyclin-dependent kinase inhibitor 1 (p21), which is a general inhibitor of CDKs, including CDK2, CDK4, and CDK6 [50]. These three CDKs suppress the action of the retinoblastoma protein $\mathrm{pRB}$ (encoded by the RB1 gene in mammals), which is the key "check-point" protein for the cell cycle to proceed from the $G_{1}$ phase. Now the transcription factor E2F family (such as E2F1) leads to transcription of genes related to the $G_{1}-S$ phase progression. The pRB protein inhibits the function of E2F. Following through the logic of activation and inhibition, we learn that activation/overexpression of p14(ARF) and p16(INK4A) would result in cell cycle arrest. Thus the two "products" of the gene CDKN2A stops cancer growth. The mutated gene CDKN2A is inactive in its function as the cell cycle check-point [51]. Thus we can summarise the above results and represent a flow chart in Figure 4 for the action of the anti-cancer gene CDKN2A.

\subsection{Methylation of the CDKN2A Gene Is Correlated with a High Risk of Pancreatic Cancer, and Inhibitors of CDK4/6 Are Looked for-We Pay Attention to Abemaciclib}

There is another type of mutation that frequently occurs in the pathway discussed. First, we note that DNA methylation is well known by now to be a process by which methyl groups are added to certain sites of the DNA molecule. Methylation can change the activity of that DNA segment without changing the sequence. When methylation occurred in a gene promoter, repression of transcription of that gene occurs. Using meta-analysis methods, based on samples from 45 chronic pancreatic patients, 155 pancreatic intraepithelial neoplasia (PanIN) patients, and 418 pancreatic cancer patients, the result in [52] indicates that methylation of the CDNK2A is correlated with a high risk of pancreatic cancer. The degree of methylation of CDNK2A promoter has been proposed as a prognostic marker of pancreatic carcinogenesis. 


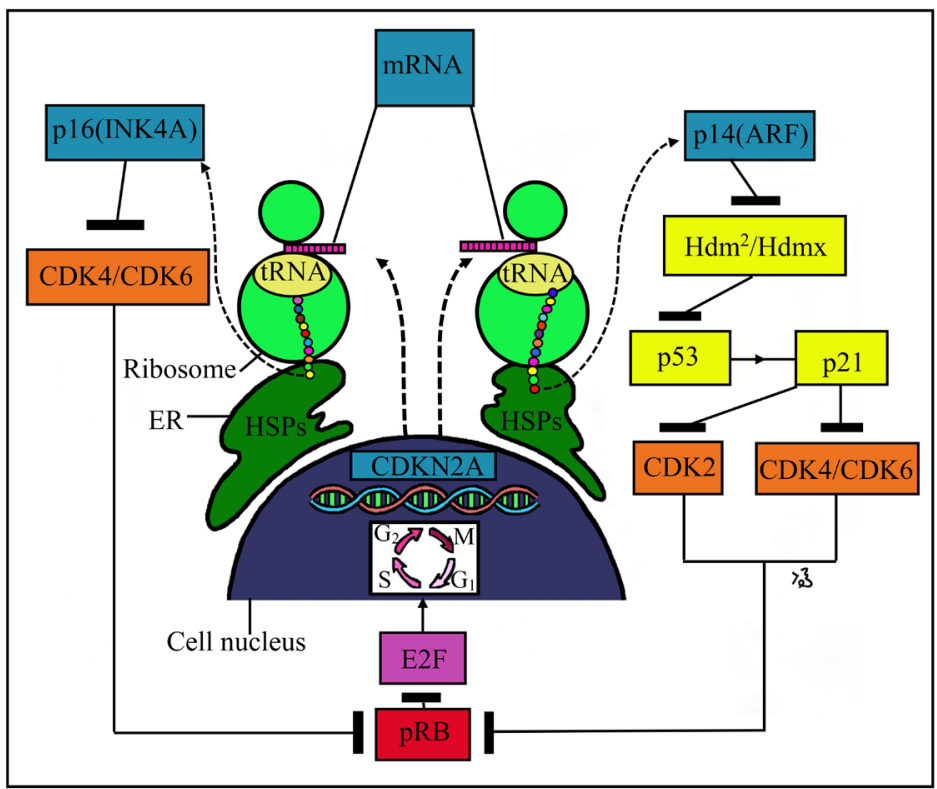

Figure 4. The CDKN2A pathway. The gene CDKN2A can transcript two variants. The messenger RNA of one variant p16(INK4A) synthesizes the protein bearing the same name via the ribosome and the help of transmission RNA (tRNA) as shown on the left side. The product protein inhibits the cyclin-dependent kinases (CDK4 or CDK6) which drives the cell cycle, particularly the $G$ phases. The other variant of CDKN2A leads to the generation of another protein p14(ARF) similarly, as shown on the right side. On the other hand, the Hdm2 forms a complex with Hdmx. This activated complex inhibits the function of p53 (a cancer suppressor) which activates the cyclin-dependent kinase inhibitor 1 (p21). Now p21 inhibits the actions of all CDKs, including CDK2, CDK4, CDK6. These three CDKs suppress the action of protein pRB, the key "check-point" protein for the cell cycle to proceed from the G1 phase. Now the transcription factor E2F is the one related to the G1-S phase progression. Following through the logic of activation and inhibition, we learn that activation/overexpression of p14(ARF) and p16(INK4A) would result in cell cycle arrest. For other details, see text. This figure was painted by author PCWF.

Based on the discussion in the above two subsections, clinicians are looking for suitable CDK4/6 inhibitors to treat a broad band of solid cancers, including pancreatic cancer. Of note, the CDK4/6 inhibitor Abemaciclib has received approval from FDA in 2017 to treat hormone receptor (HR)-positive, human epidermal growth factor receptor 2 (HER2)-negative advanced/metastatic breast cancer according to [53]. Last year, the same drug has been applied to treat pancreatic cells with efficacy. Whether this drug can be put on clinical trial, we believe the situation should become clear within one year or two [54].

\subsection{Iron Chelators as Potential Anti-Cancer Drugs-The Compound DD44mT, as an Example, Promoted Activity of p21 in the CDKN2A Pathway in Figure 4}

We note that more than a decade and a half ago, to look into the mechanism of interaction of Fe chelator with gene expression, gene arrays were used to assess gene expression after incubating cells with two Fe chelators, 1) desferrioxamine 
(DFO) and 2) 2-hydroxy-1-naphthylaldehyde isonicotinoyl hydrazone (abbreviated as 311) [55]. In that analysis, only the $\mathrm{N}$-myc downstream regulated gene 1 (NDRG1) was specifically up-regulated by Fe chelation. Such up-regulation was mediated by hypoxia-inducible factor-1 (HIF-1)-dependent and -independent mechanisms [55]. Several months ago, the cancer suppressor function mechanism of Ndrg1 has been found to participate in the activation of p21 (effectively upregulation of p53) [56].

On the other hand, over 2 decades ago, detection of the expressions of Ndrg-1 in breast epithelial cells as well as breast cancer cells, it was pointed out in [57] that Ndrg-1 participated in cell cycle progression.

Using two human colorectal cancer cell lines HCT116 p53+/+ \& HCT116 p53-/- in the study of [58], a detailed analysis suggested that Ndrg-1 is necessary for p53-dependent apoptosis. Following that, NDRG1 upregulation in pancreatic cancer has been found to be correlated to a significant reduction in primary tumor growth, angiogenesis, and metastasis [59]). In the same year, the Fe chelator, di-2-pyridylketone 4,4-dimethyl-3-thiosemicarbazone (Dp44mT) with selective antitumor activity was reported to upregulate NDRG1 in vitro and in vivo in many tumor types, including pancreatic cancers [60].

Later, looking into the mechanism of interaction between NDRG1 and (Dp44mT), The Fe-chelator (DD44mT) hydrochloride was reported in [61] to activate NDRG1 by phosphorylation at the Ser330 and Thr346 sites which are relevant to cancer cell proliferation. Moreover, the Fe chelator increased expression of the cyclin-dependent kinase inhibitor p21 (inhibiting CDK2, see Figure 4), whereas decreasing cyclin D1(an effector that regulates cell proliferation by blocking cells at G1 phase) was observed in pancreatic cancer cells. The growth of the pancreatic cancer xenografts was also found to be inhibited by the above Fe chelator, without causing alterations in normal tissue histology [61].

\section{New Discoveries of the Hippo Signaling Pathway in Pancreatic Cancer Progression}

\subsection{Some Basics of the Hippo-YAP Signaling Pathway Relevant to Carcinogenesis of Pancreatic Cancers}

Classically, the Hippo pathway is a system of signaling effectors serving as a checkpoint for proper exit from mitosis. The basic function is conserved through evolution, but with some replacements of proteins participating in different species and cell types within the same species [62]. The initial stimulating mechanism is still not fully understood in the human pancreas, but let us introduce some key proteins in the Hippo signaling pathway. The first two key members are: YAP1 (yes-associated protein 1, also known as YAP or YAP65), and TAZ (transcriptional co-activator with a PDZ-binding domain; also known as WW domain-containing transcription regulator 1, or WWTR1). The formal name of the protein encoded by TAZ is Tafazzin, but TAZ is used commonly for both the gene and the protein in literature now. YAP and TAZ do not have DNA-binding 
domains, and they are transcriptional coactivators. In normal cells, they are mainly found in the cytoplasm. Upon being translocated into the nucleus, they regulate gene expression through interaction with TEAD1-4 (transcriptional enhanced associate domain protein, with members labelled 1-4), which mediate the main transcriptional output of the Hippo pathway in mammalian cells [63].

In the "un-stimulated state of the Hippo pathway", protein TEAD can also bind to VGLL4 (vestigial-like proteins with four members 1-4) in the nucleus and thus function as transcriptional repressors. VGLL1-4 does not contain a DNA-binding domain and they also impose their transcriptional regulatory functions through binding with TEADs [64]. Though the study pertains to gastric cancer, since the Hippo pathway is conserved in the downstream signaling, such TEAD-VGLL4 interaction holds for a variety of cells. There is no transcription due to the Hippo pathway and the proliferation of the cell is checked at that state (as schematically shown in Figure 5(a)), so far as YAP/TAZ is kept in the cytoplasm, because YAP/TZ competes with VGLL to bind TEAD in the nucleus.

On the other hand, under physiological growth conditions, p53 is maintained at a low level by the E3 Ubiquin ligase Mdm2, which together with its family member Mdm4 (in a complex form), mediates partial p53 proteasomal degradation. Some amount of p53 remains in the cytoplasm [65]. Also, the enzyme Large tumor suppressor kinase 1 (LATS1, with LATS2, LATS3, LATS4 in the family) has been found to inhibit both the action of translocation of YAP/TAZ, as well as the action of protein $\mathrm{Mdm} 2$ in its negative regulation of $\mathrm{p} 53$.

\subsection{Agonists of All the Three Proteins-p53, Ptpn14, LATS (Members 1-4), Inhibitors of Furin Are Potent Future Drugs to Treat Pancreatic Cancers Basing Their Inhibition of Translocation of YAP/TAZ to the Nucleus}

There are many proteins involved in holding YAP/TAZ in the cytoplasm, depending on cell type, and we shall only bring in the up-stream signaling involving the gene called PTPN14 [66] which encodes the protein called "protein tyrosine phosphatase non-receptor type 14" (Ptpn14). This protein contains an $\mathrm{N}$-terminal noncatalytic domain like that of band 4.1 superfamily cytoskeleton-associated proteins, suggesting that this protein is located in the cytoplasm or cell membrane. In literature, Ptpn14 or PTPN14 has been used to represent this encoded protein.

To briefly describe how p53 would regulate Yap, we need to define the term "TAD", an abbreviation of "topologically associating domain". TAD is a self-interacting genomic region, implying that DNA sequences within a TAD physically interact with each other more frequently than with sequences outside the TAD. There are two TADs found in the gene TP53 [67]. This finding is relevant to the mutations in p53 in the experiment on the analysis of the Hippo pathway specified below.

Note that a knockin mouse is an animal model in which a gene sequence of interest is altered by one-for-one substitution with a transgene, or by adding 
gene sequences that are not found within the locus. In a comprehensive study of the Hippo pathway in [68], p53 knockin mouse strains expressing mutants in p53 TADs were prepared according to the technicality described in [69]. Several human and animal (non-pancreatic) cancer cell lines together with human PanIN and PDAC cells, were included in this study. The authors of [68] have found that 1) p53 negatively regulates Yap through Ptpn14 activation; 2) p53-Ptpn14-Yap is a key "tumor suppressive axis"; 3) genes TP53 and PTPN114 mutations are mutually exclusive in human cancer.

Moreover, the ubiquitously expressed serine protease Furin can cleave a plethora (excessive) of proteins at polybasic recognition motifs [70]. Using five human pancreatic cancer cell lines, it has been demonstrated that depletion of the protein Furin would result in a significant reduction of EMT formation, cell proliferation, migration, and invasion of these cancer cells [71]. Thus inhibitors of Furin deserve attention as a therapeutic measure to PDAC.

Summing up the rather sophisticated in vitro and in vivo analysis (of the mouse model [68]), together with the works of other groups on the Hippo pathway [64] [70] [72], we present a summary in Figure 5(b), the main part of which is based on Figure 7(E) of [68]. In this figure, the Kibra protein is encoded by gene WWC1 and has been inferred to be a negative regulator of the Hippo pathway [73]. In the work of [68] Kibra seems to provide a means of cross-talk between LATS and Ptpn14, both inhibiting the translocation of YAP/TAZ to the nucleus. If either TP53 or PTPN14 is mutated, there is a high chance of translocation of YAP/TAZ to the nucleus. From the clinical point of view, we propose that antagonists of all the three proteins-p53, Ptpn14, LATS (members 1-4), inhibitors of Furin should be considered in further experiments in vitro and in vivo, for the treatment of pancreatic cancers. The potent inhibitor of Furin, i.e., phenylacetyl-Arg-Val-Arg-4-amidinobenzylamide (15) has been synthesized in the laboratory [74]. The interaction between YAP/TAZ and TEAD1-4 dissociates VGLL4 from TEAD1-4 and thereby activates TEAD-mediated gene transcription to promote tissue growth and inhibit apoptosis.

The c-Jun N-terminal kinases (JNKs) have three members; JNK1 and 2 are found in all tissues so far known, and JNK3 is found in the brain. Using the transgenic mouse model [75], it has been shown that the mere activation of JNK would induce insulin resistance in pancreatic $\beta$-cells by inhibition of insulin signaling in these cells, but it is not sufficient to evoke $\beta$-cell death. On the other hand, whether JNK plays a role in the carcinogenesis of acinar cells is not clear. In another study reported in [76], using human mammary epithelial cell line MCF10A, when cultured in a soft substrate, protein molecules YAP were mainly located in the cytoplasm, whereas YAP molecules were predominately in the nucleus when the cells were on a stiff substrate. Analyzing the expressions of 1) the proteins JNK, 2) LIMD1(LIM domain-containing protein 1, known to regulate negatively the Hippo signaling pathway and to antagonize phosphorylation of YAP1, 3) LATS1, which take part in the Hippo signaling pathway, it has been 
concluded that mechanical regulation alone could translocate YAP to the nucleus via the JNK-mediated inhibition of the Hippo signaling process [76]. In passing, we should note that mechanotransduction has already been reviewed as the upstream triggering signal of the Hippo pathway for cells apart from that of the pancreas [62].

Despite the convincing verification of mechanotransduction being able to trigger translocation of YAP in epithelial and other cells, whether mechanical stimulation at the cell membrane in pancreatic cells is still under research, and we put a question mark in Figure 5(b), a mechanism to be filled in hopefully in the near future.

\subsection{Discovery of Synthetic Anti-Cancer Compounds SR1078 That Stabilizes p53 and Naphthofluorescein Disodium Salt That Inhibits the Action of Furin}

In 2012, a novel mechanism for the stabilization of p53 protein expression via activation of the orphan nuclear receptor-RORa was introduced [77]. It was demonstrated, employing a human liver cancer cell line HepG2, treatment of synthetic ROR agonist, SR1078, would lead to p53 stabilization and induction of apoptosis. Note that orphan receptor $\alpha(\mathrm{ROR} \alpha)$ is retinoid-related. Several months ago, gastric cancer cell line MGC803 with RORa-overexpressing and silencing were constructed (for details see Figure S1B, C) of [78]). It has been found that overexpression of RORa could inhibit the proliferation, EMT, and invasion of these cells [78]. It has been pointed out that stimulation of G-protein-coupled receptors by glucagon or epinephrine activates Lats1/2 kinase activity, thereby inhibiting the YAP function [79]. More recently, the drug AICAR (5-Aminoimidazole-4-carboxamide ribonucleotide) and its monophosphate derivative ZMP has been shown to be agonists of LAT1,2, producing anticancer effects [80].

Note that an analog of adenosine monophosphate (AMP) can stimulate AMP-dependent protein kinase (AMPK) activity (see Figure 2). AICAR has already been used clinically to treat ischemic heart disease [81]. Collagen degradation enzymes and their inhibitors have been implied in carcinogenesis in general. By 2000, however, phase III clinical trials of MT1-MMP inhibitors demonstrated no clinical efficacy in treating cancers (see review of [82]). A revisit to inhibitors of Furin was carried out in 2008 [83]. It has been found that Furin would play role in processing the protease membrane type-1 matrix metalloproteinase (MT1-MMP) and in enhancing cellular motility and invasiveness of COS cell line (fibroblast-like cell lines derived from monkey kidney tissue) and human HT1080 cancer cell line (a fibrosarcoma cell line). A small molecule inhibitor of Furin, which is a naphthofluorescein disodium salt (B3), is a mixture of dihydroxynaphthalene and phthalic anhydride. This salt was prepared and found to inactivate the proprotein convertases of Furins, and lead to anti-invasive effects of the two cell lines stated above [83]. 
(a)

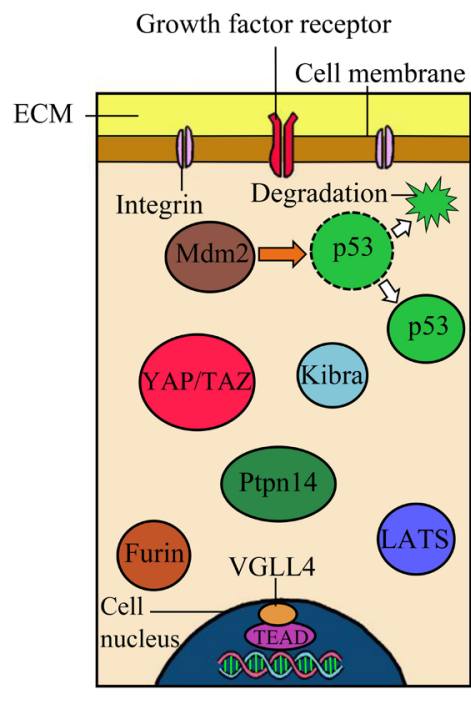

No transcription resulting from Hippo pathway (b)

If Furin or Mdm2 is overexpressed, $\mathrm{YAP} / \mathrm{TAZ} \rightarrow-\rightarrow$ nucles

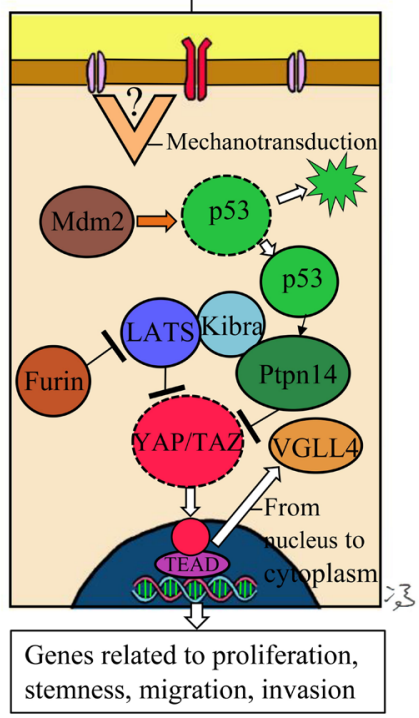

Figure 5. During the normal state, protein Mdm2 induces some of the p53 molecules to go through proteasomal degradation, retaining part of it in the cytoplasm. by proteasomal degradation. VGLL4 does not contain DNA-binding domain, and when it binds with TEAD, there is no transcription resulting from the Hippo pathway as shown schematically in (a). Now p53 protein is a strong activator of Ptpn14, which forms a complex with LATS and Kibra. Both Ptpn14 and LATS are inhibitors of YAP/TAZ (one of the few key proteins in the Hippo pathway), so that YAP/TAZ cannot be translocated to the nucleus. However, if i) p53 is mutated, or ii) there is overexpression of Mdm2, or iii) protein Furin, which is an inhibitor of LATS, is activated, there are situations where YAP/TAZ can no longer be held in the cytoplasm; YAP/TAZ has a strong affinity to the transcriptional factor TEAD and will be translocated to the nucleus, replacing VGLL4, and transcription of genes related to the box below (b) occurs. For pancreatic cancers, mutation of gene TP53 has been found to be correlated to a high percentage of carcinogenesis. Whether the upstream triggering factor arises from mechanotransduction is still under research, but there is evidence that at least for epithelial cells, mechanical stimulation can trigger the translocation of YAP/TAZ via the JNK route. For details, see text. This figure was painted by author PCWF.

\section{Over $50 \%$ of the Pancreatic Cancer Cells Have Mutation (Loss of Function) of the Gene SMAD4, and It Is Important to Look for Measures to Activate This Gene}

\subsection{Transforming Growth Factor- $\beta$ and the SMAD Pathway}

The canonical transforming growth factor-Smad (TGF- $\beta$-Smad) signaling pathway is initiated by activation of the transforming growth factor receptor (TGF- $\beta$ R) by its ligand TGF- $\beta$, as shown in Figure 6 . The activated Smad complex is heterotrimeric, composed of two phospho-Smad2 or phospho-Smad3 moieties with a Smad4 molecule to become a site specific transcriptional regulator. Thus, we express the number $2 / 3$ in the "R-Smad pair". This "Smad transcriptional regulator" recruits coactivators or corepressors to regulate target gene expression. For normal cells, the proteins generated via the "canonical 
Smad pathway" cause cell cycle arrest, inhibition of immortality, and allow apoptosis. Therefore, activation by TGF-Smad represses carcinogenesis [84]. TGF- $\beta$ is normally dormant in the ECM and a special mechanism is needed for its release. In this section, we leave out the mechanism on such release, as it will be analyzed in a later section. We start simply by considering the "canonical pathway" initiated by binding of the TGF $-\beta$ (as a ligand) to its receptor(s). The receptor TGF- $\beta$ R is built of two dormant units of type I and type II receptors: TGF- $\beta$ RI and TGF- $\beta$ RII. A TGF- $\beta$ ligand "joins" these two units on docking from the extracellular domain, leading to phosphorylation of the type I receptor (Figure 6(a)). The combination of (TGF- $\beta$ with TGF- $\beta$ RI and TGF- $\beta$ RII) can be treated as a complex [85].

On the other hand, the protein SarA (or called endosomal protein) interacts directly with the Smad2/3-complex of proteins in the cytosol and recruits it to the activated TGF- $\beta$ receptor complex stated [86] (Figure 6(b)). The complex $(\mathrm{Smad} 2 / 3)$ is phosphorylated and leaves the receptor site, attracting the common mediator Smad4 (a well-established process) [87]. The enlarged complex of (Smad4 - Smad2/3) is then translocated to the nucleus; with the help of a transcription factor targeted for a particular gene (plus the help of other supporting proteins), transcription proceeds (Figure 6(c)), leading to the synthesis of the associated protein in the cytosol. The protein SarA is degraded, after completing its work. We follow up on the proteins synthesized by this pathway and the effects on carcinogenesis in the next section. Note that TGF- $\beta$ s signaling regulates the transcription of over 500 genes.

\subsection{Evidence of the Anti-Cancer Signature of the TGF- $\beta$-Samd 4 Signaling Pathway-The Roles of Genes PAI-1, AKAP12, and ITGB6}

A large-scale microarray approach has been used [88] with an inducible system to knock down levels of Smad4 in either the human immortalized keratinocyte cell line HaCaT or a pancreatic tumor cell line, Colo-357. Out of the 114 TGFtarget genes, 49 were identified as Smad 4 dependent and 65 were Smad 4 independent. To validate the screen, RT-PCR and RNase protection analysis of representative genes were then carried out and it was reported that at the mRNA level, 1) PAI-1,2) AKAP12 (protein Akap12), and 3) ITGB6 were confirmed to be Smad4-dependent, TGF-target genes. First, let us analyze the functions of the proteins encoded by these three genes.

Plasminogen activator inhibitor-1 (PAI-1) is one of the key regulators of tumor invasion and metastasis. SW1990, which is a human highly metastasis pancreatic cancer cell line, was transfected with the protein PAI-1(called SW/PAI-1 cell line) in the study of [89]. Cellular invasion, in vivo tumorigenesis in xenograft and liver metastasis, were found to be significantly suppressed in SW/PAI-1 cells when compared to control cells. Determination of the microvascular density and the change of expressions in the key angiogenesis factor VEGF as well as TGF- $\beta 1$, it was concluded that one crucial function of PAI-1, 
and hence Smad4, would be suppression of angiogenesis (i.e. with anti-cancer action).

The gene AKAP12 encodes protein A-kinase anchor protein 12 (Akap12) which has the function of anchoring protein that mediates the subcellular compartmentation of protein kinase A (PKA) and protein kinase C (PKC). Protein kinase A regulates glycogen, sugar, and lipid metabolism. PKC is a family of protein kinase enzymes that are involved in controlling the function of other proteins through the phosphorylation of hydroxyl groups of serine and threonine amino acid residues on these proteins [90]. In addition to binding PKA and PKC, AKAP12 also binds other signaling mediators such as calmodulin, F-actin, cyclins, Src (a non-receptor protein tyrosine kinase that is involved in the control of cell adhesion, growth, movement, and differentiation). Reduced AKAP12 expression has been consistently observed in liver cancer cell lines [91], and based on its ability to suppress growth rates and promote reorganization of the actin-based cytoskeleton in transformed fibroblasts (as reviewed in [92]), it has been classified as a class II tumor suppressor gene (in general).

With human liver cancer cell lines expressing AKAP12 and/or miR-103 (micro RNA which targeted AKAP12), the anti-cancer nature of AKAP12 was further confirmed in liver cancer. Without detailed data of AKAP12 related to pancreatic cancer at this moment, we accept the microarray analysis (of pancreatic cancer cell line) of [88] and propose that AKAP12 would be an anti-cancer protein depending on the TGF- $\beta$-Smad4 pathway in the pancreatic cancer too. The gene ITGB6 encodes the protein integrin $\alpha_{v} \beta_{6}$ [93] which takes part in mediating RGD-dependent release of TGF- $\beta 1$ from the dormant Latency-associated peptide (LAP), thereby initiating the TGF- $\beta 1$-Smads signaling pathway. Thus, activation of Samd4 leads to the synthesis of proteins that play roles in anti-cancer action. We enter the synthesized proteins in the right-hand lower corner of Figure 6.

Over a decade ago, sequencing was carried out in more than 750 million base pairs of DNA from 23, 219 transcripts in a series of adenocarcinomas of the pancreas from 89 patients who underwent pancreaticoduodenectomy. The result indicates that patients with SMAD4 gene inactivation survived a median of 11.5 months, compared with 14.2 months for patients without SMAD4 inactivation [94]. Though the improvement is not large, it supports the notation that inactivation (loss of function) of the gene SMAD4 is a cause of carcinogenesis.

\subsection{Attenuation of Cancer Stem Cells' Tumorigenic Ability by TGF- $\beta$-Smad4 Signaling}

In the study of [95], three human pancreatic cell lines were used: 1) Panc-1, 2) BxPC-3, 3) SUIT-2. RT-PCR analysis revealed that SMAD4 mRNA and Smad4 protein were expressed in 1) \& 3). The 4) cell line was constructed by knocking down SMAD4 by shRNA in 1). Cells 1) cultured with shNTC is named cell line 5). Using "RNA knockdown technology", the term shNTC stands for nontarget control (NTC) short hairpin (sh) shRNA. Cells of line 4) were injected into mice 
to create the (a) xenograft model group. Cells of line 5) were also injected in the mice model to create the control in vivo group, called group (b). The tumors generated in group (a) were found to be larger than those of group (b). It was reported that expressions of cyclin-dependent kinase regulators (such as CDKN1A, CDKN1B, CDKN2A, CDKN2B, MYC) were not different in cells 4) and 5), it was inferred that Smad4 did not affect the cell cycle progression of the cancer cells.

Based on the information that the loss of function of Samd4 occurs in most PDAC, and the established evidence of participation of cancer stem cells in tumorigenesis (evidence will be presented in a later section), authors of [95] looked for markers of the cancer stem cells, though CD44 was known to be one. There was an experiment showing aldehyde dehydrogenase type 1,2,3 $(\mathrm{ALDH} 1,2,3)$ were also such markers of the stem cells [96]. The isoform ALDH1 was nailed down as more specific and pancreatic cancer cells of the cell lines used were found to contain $3 \%-10 \%$ of ALDH1+ cells. The regulation abilities of

(a)

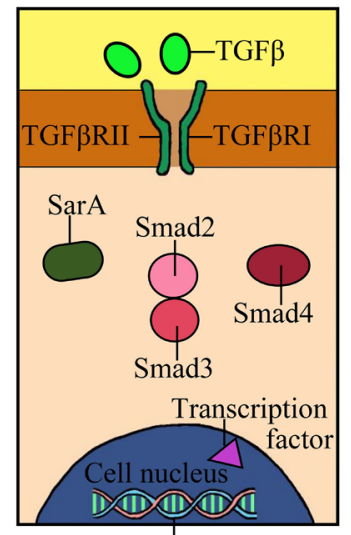

DNA

No transcription from the TGF $\beta$-Smads pathway yet

(b)

DOIIIIIII

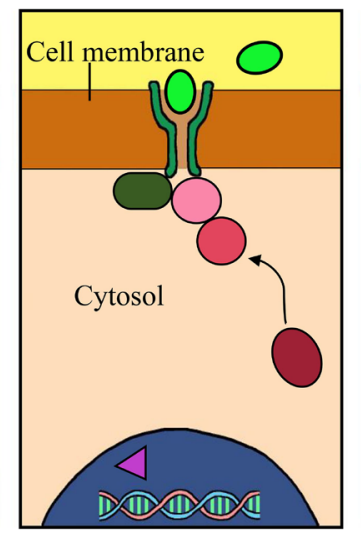

Transcription of PAI-1,
AKAP12, ITGB6, leading
to proteins inhibiting cancer
promoting interactions

Figure 6. The canonical transforming growth factor- $\beta$-Smad pathway. TGF- $\beta$ (bright green) $=$ transforming growth factor $\beta$. TGF- $\beta$ RI, TGF- $\beta$ RII (both deep green) $=$ transforming growth factor $\beta$ receptors type I \& II respectively. SarA (greyish) $=$ Smad anchor for receptor activation. The Smads (with 8 members so far known) are a group of intracellular proteins specific for transmitting signals from TGF $\beta$ superfamily at the cell surface to the nucleus. When the receptors TGF- $\beta$ RI, TGF- $\beta$ RII are activated by TGF- $\beta$, protein SarA is recruited from the cytosol, resulting in phosphorylation of first TGF- $\beta$ RII and then TGF- $\beta$ RI. The complex Smad2/Samd3 (light and medium red respectively) is also recruited and phosphorylated, attracting the "co-Smad mediator, or Smad4 (deep red)" also, while SarA leaves and be degraded. The bigger complex Smad2/3/4 is translocated to the nucleus. With the help of the specific transcription factor (light purple), transcriptions of specific genes PAI-1, AKAP12, ITGB6 lead to the synthesis of proteins inhibiting cancer-promoting pathways (for details see text). Bone morphogenetic proteins (BMPs) are a group of growth factors that also trigger the TGF- $\beta$-Smad pathway, but with other Smad members participating. This figure was painted by author PCWF. 
TGF- $\beta$ and bone morphogenetic protein-4 (BMP-4) on ALDH1 activity were also carried out. The key discoveries were: 1) ALDH1 was enriched only in cancer cells with SAMD4 knockdown. 2) ALDH1 was necessary for the tumorigenic ability of pancreatic cancer cells. 3) TGF- $\beta$ triggered signaling involving Smad4, attenuated tumorigenic ability of ALDH1(high) cancer cells, and (BMP-4) triggered signaling was much less potent in such attenuation. The overall result is that TGF-Smad4 signaling would suppress cancer stem cells' tumorigenesis.

Note also that activation of the TGF $\beta$-1-Smad has cross talks with other cancer-promoting pathways Ras-Raf-Mek-Erk for cell proliferation, and the Rho-Rock pathway (helping Actin chains formation) for migration and invasion, and we will not discuss such interactions in this paper. Information on this, and the last section gives a clear explanation of the reason why the loss of functions Smad4 would promote carcinogenesis, as observed clinically.

\section{The EGF-Jak-STAT Signaling Pathway and the Retrograde Transport of EGFR to the Nucleus}

\subsection{The Epidermal Growth Factor EGF Can Induce Pancreatic Carcinogenesis via the Jak-STAT Pathway}

Growth factors are secreted by cells to the interstitial fluid and some are stored in the ECM whereas some find their destinations-their receptors directly. The ones that are in the dormant states in different comparts of the fascia can be released from the ECM by mechanical force/chemical force [2] [37] under specific conditions with an example described in detail in a later section. On reception of these signals (ligands) by the growth factors receptors, specific signaling pathways are in action, some of which have been described in the last few sections. The epidermal growth factor (EGF), the transforming growth factor (TGF) are two key growth factors regulating many cellular functions. For example, through EGF's binding to cell surface receptors, EGF activates an extensive network of signal transduction pathways that include activation of the PI3K/Akt and Ras/Erk pathways, as already described in detail in Sections (3) and (4). These pathways predominantly lead to activation or inhibition of transcription factors that regulate expressions related to the synthesis of proteins for proliferation or apoptosis. Off-balance of the expressions of key genes/proteins would lead to pathological state(s). Mutation (aberrant activation) of genes responsible to synthesize growth factor EGF is often detected during carcinogenesis [97].

Four tyrosine kinase transmembrane receptors constitute the EGF receptor family: 1) ErbB1/EGFR, 2) ErbB2/HER2/neu, 3) ErbB3/HER3, and 4) ErbB4/HER4. EGF binds exclusively with ErbB1/EGFR; EGF activates the Janus kinase (Jak 1 or Jak2) of the Jak-STAT signaling pathway. Here Stat stands for signal transducer and activator of transcription protein. The Janus kinase (Jak) is a family of (four) intracellular, nonreceptor tyrosine kinases (Jak1, Jak2, Jak3, Tyk2). Upon IL-6 binding to its receptor called gp130 (a transmembrane glycoprotein), the activated IL-6-gp130 system/complex participates in phosphorylation of Jak protein. The Jak-STAT signaling pathway is therefore also called the IL-6-Jak-STAT 
signaling pathway in some literature. For simplicity, we leave out the part played by gp130 and IL- 6 in Figure 7 below. We simply consider that two Jak1 proteins are attracted to the (activated) receptor ErbB1, giving up two phosphates (Figure 7(a)). Two "signal transducer and activator of transcription proteins" (called STATs, with label 1 to 6) are attracted to the intracellular part of the receptor, accepting the phosphates (Figure 7(b)).

In doing so, they form a dimer and is translocated to the nucleus after passing through the nuclear pore complex (Figure $7(\mathrm{c})$ ). Again, some factors (represented by one transcription factor, the purple triangle in the figure) together with the STAT1 dimer triggers transcription of genes and eventually synthesis of proteins related to proliferation, cell cycle progression, immunity. Note that a gene called suppressor of cytokine (SOC) is also expressed, leading to the corresponding protein which inhibits the dimerization of the STAT proteins and hence the Jak-STAT pathway, as a negative regulation. In this pathway, the receptor remains in the cell membrane. There is another mechanism where EGFR is translocated to the nucleus in a complicated way; this process is described in the following section.

(a)

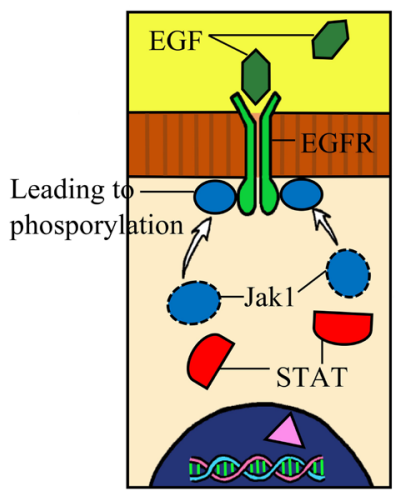

No transcription (b)

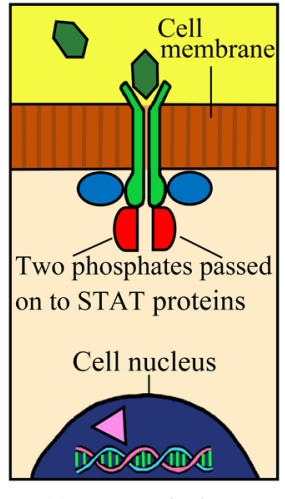

No transcription 冯 (c)

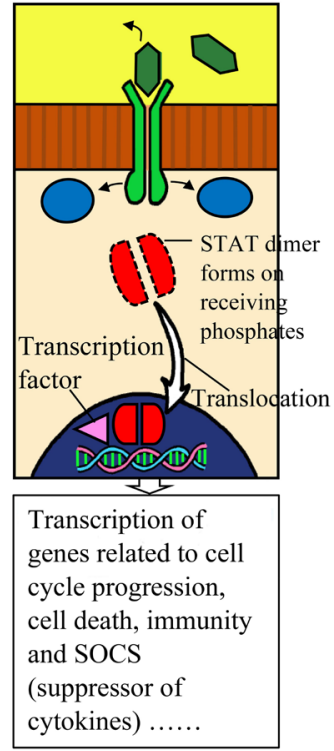

Figure 7. The Jak-STAT pathway was triggered by the epidermal growth factor (EGF). On reception of the EGF, the EGF receptor is phosphorylated, attracting two Jak1 molecules to the internal side of the cell membrane, as shown in (a). Two phosphates are passed on from the receptor complex to two STAT proteins in the cytosol (b) and the STATS form a dimer; such binding allows them to leave the cell membrane domain and the dimer is further translocated to the nucleus, passing through nuclear pore complex, where some proteins participate in helping the transcription of specific genes. (c) The light purple transcription factor represents a group of the stated transcription proteins. Apart from the usual genes leading to synthesis of proteins related to cell growth, differentiation, immunity response, a gene called suppressor of cytokine (SOC) is also expressed, as a (negative) regulator of the dimerization of the STAT proteins. For other details, see text. This figure was painted by author PCWF. 


\subsection{The Dynein Motor and Retrograde Transport of the EGFR to the Cell Nucleus}

In many physiological processes, proteins are synthesized and transported from the ER to the cytoplasm, cell membrane, and some of them might be secreted to the ECM by exocytosis. Such trafficking is called anterograde transport. In contrast, many cell-membrane growth factor receptors (together with the simulating growth factors) have been observed to be translocated to internal departments of the cell first by endocytosis, which is characterized by membrane and vesicular trafficking [98].

Before we describe the mechanism further, we need to introduce the vehicle (like a car, called a molecular motor) which brings the receptor along. Molecular motors are machines that convert free energy, mainly obtained from ATP hydrolysis, into mechanical work. It has been pointed out that the cytoskeletal motor proteins of the myosin and kinesin families, which interact with actin filaments and microtubules, respectively, would lead to intracellular cargo transport [99]. A typical molecular motor is a member of the superfamily of dynein; it is a complex composed of two heavy chains (deep red in Figure 8), three intermediate chains (also red), and four light intermediate chains (orange) [100].

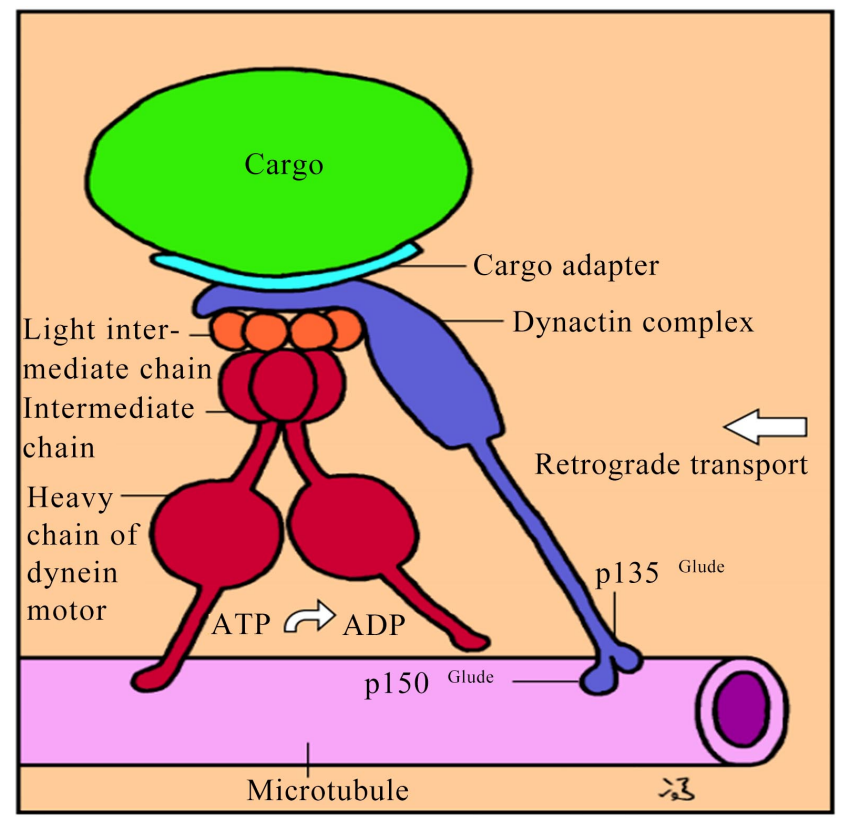

Figure 8. The dynein motor protein is built of two heavy chains, three intermediate chains and four light intermediate chains. The dynactin complex (bluish purple) has 10 subunits as described in the text. The part of the dynactin attached to the dynein protein also attracts a suitable cargo adapter protein (light blue) for a specific cargo (green), such as an epidermal growth factor receptor set. While ATP is converted into ADP in the dynein motor, energy released is converted to mechanical work to transport the motor set with the cargo towards the nucleus direction. The light purple tube is a simplified form of a microtubule. There are many microtubules to keep the integrity of the cell shape. Such a transport towards the nucleus is called retrograde transport in the cytoplasm. This figure was painted by author PCWF. 
Each of the two heavy chains has an extension with a binding site attached to a microtubule. To transport a cargo, each dynein must be associated with another complex called dynactin (bluish-purple). The dynactin complex has ten subunits-p150 ${ }^{\text {Glued }}$, p135 ${ }^{\text {Glued }}$, p.62, dynemitin (p50), actin capping protein $\beta$ subunit, $\mathrm{p} 27$, and $\mathrm{p} 24$. The heterodimer formed by ( $150^{\text {Glued }}-\mathrm{p} 135^{\text {Glued }}$ ) is a side-arm of the dynactin complex, ending with two spherical-shaped heads each of which contains a microtubule binding site [100]. This complex is painted with a simplified structure. The dish-like part (light blue) attached to the "top part" of the dynactin complex is an adapter protein for a specific cargo (green), such as an epidermal growth factor receptor set (EGF plus EGFR). While ATP is converted into ADP in the dynein motor, the energy released is converted to mechanical work to transport the motor set with the cargo towards the nucleus direction.

Microtubules are microscopic hollow tubes made of the protein $\alpha$ and $\beta$ tubulin (around $24 \mathrm{~nm}$ in diameter), forming a network of protein filaments that extends throughout the cell, maintaining the cell shape. They have roles in cell movement, cell division, and intracellular trafficking of proteins/peptides. During the past several years, there have been compiling evidence of such "on the micro-tubule" transport of various types of cargoes (such as vesicles containing proteins, membrane receptors, mitochondrion), both away from the nucleus (anterograde transport) and towards the nucleus (retrograde transport).

\subsection{Retrograde Transport of EGFR in Action}

During the recent several years, we begin to understand more about the retrograde transport of cell membrane receptor. Now refer to Figure 9 on retrograde transport of the EGFR set. At site (1), the epidermal growth factor (EGF, dull green) docks at its cell membrane receptor EGFR (also green, but brighter). Upon activation by EGF, the receptor together with the growth factor can be engulfed by the cell by the common endocytosis process (as represented by position (2) in Figure 9).

Using Hela cells, after endocytosis, the EGFR-set enclosed by the vesicle, has been found to be carried by the dynein motor protein (with Dynactin and cargo adapter) on route to the Golgi apparatus along a microtubule, as demonstrated by immune-microscopy investigation with gold particles, as well as RNA detection in the Golgi in the investigation of [98]). This part of the transport is represented by site (3), where the dynactin and cargo adapter protein have been neglected, and the motor protein dynein is represented by a figure simplified from Figure 8. The EGFR-set is enclosed by a vesicle (the brown circle in the figure). Reaching the membrane of a Golgi apparatus, a protein called soluble N-ethylmaleimide-sensitive fusion protein attachment protein receptor (SNARE), member syntaxin6 functions to transport the vesicle through the Golgi membrane by a fusion process [101]. The fusion process is also included in site (3) in the figure. The EGFR-set inside the Golgi apparatus, via a process still not fully understood, is transported to some part near a microtubule, as sche- 
matically represented by site (4).

After transporting inside the Golgi apparatus, it has been found to exit to the cytoplasm with a "coat protein complex I" (COPI) vesicle, which consists of a small GTPase ADP-ribosylation factor (ARF) and a "coatomer" (which is simply a protein complex that coats membrane-bound transport vesicles). A coatomer has seven subunits ( $\alpha, \beta, \beta, \gamma, \delta, \varepsilon$, and $\zeta$ ) [102]. Using MDA-MB-468 cells (derived from a breast cancer patient), the ARF protein with $\gamma$-coatomer called $\gamma$-COP has been identified by immunoblotting and revelation by confocal microscopy [103].

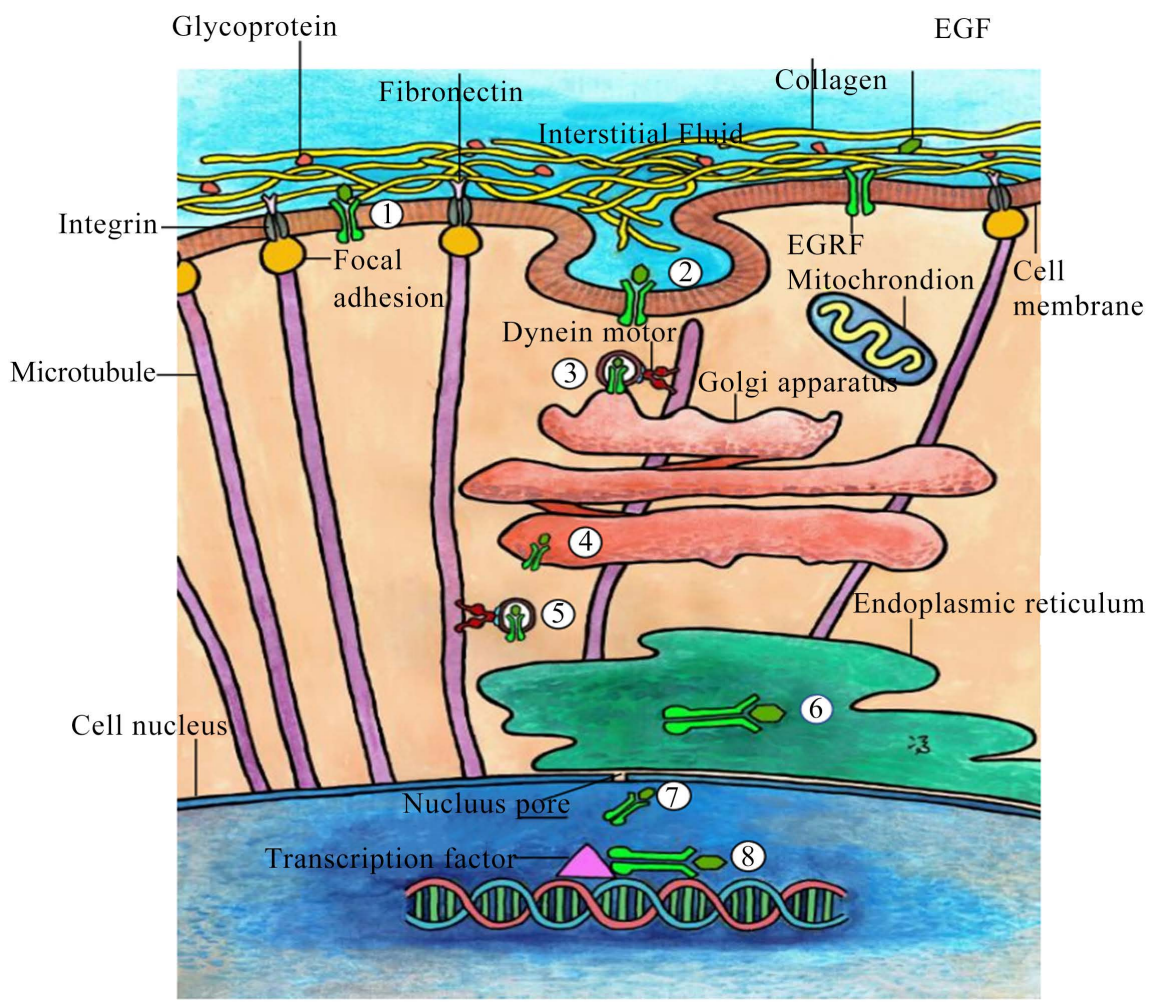

Figure 9. The retrograde transport of EGFR is set to the nucleus. At site (1), the epidermal grow factor (dull green) docks at the membrane receptor EGFR (bright green). At site (2), endocytosis encloses the EGFR-set in a vesicle (boundary was painted in brown). At (3), the dynein motor (red), with the help of the dynactin complex (omitted in this figure, see details in text and Figure 8) and the cargo adaptor (light blue) carries this cargo along a microtubule track to the Golgi apparatus. The fusion of the cargo to the Golgi apparatus is governed by a protein called "syntaxin6". The EGFR-set is transported inside the Golgi apparatus to a site near a microtubule (4). Another dynein motor carries the cargo, now enclosed by a vesicle bounded by a "coat protein complex I" (COPI) (painted in deep brown) to the endoplasmic reticulum (ER) as indicated at (5). The cargo fuses through a protein-conducting channel at the membrane of the ER, into ER (6). The cargo is translocated through a pore/slit at the nuclear external membrane, into the nucleus (7). The purple triangle represents a group of proteins participating in choosing the gene(s) specific to the EGFR-set for transcription (8). There is evidence that the EGFR-set can be translocated to the mitochondrion also. Microtubules are generated from focal adhesion complexes attached to integrins. See text for other details. This figure was hand-painted by author PCWF. 
Since EGFR sets have been found in the nucleus of a large number of cancer cell lines, it has been proposed already in 2010 [103] that EGFR is further translocated from the ER via the nuclear pore complex to the nucleus by the dynein motor with the cargo enclosed in one type of COPI vesicle. We accept this model and consider that the EGFR-set is enclosed in one type of COPI vesicle (such as $\gamma$-COP, painted in deep brown) and is transported also by the dynein motor from the Golgi to the endoplasmic reticulum (ER) in the pancreatic cells.

Following up on the picture of retrograde transport, site (5) represents the process of a dynein motor carrying a COPI vesicle with the EGF-set from the Golgi to ER. The Golgi apparatus was painted in red with shades. There are protein-conducting channels (PCC) at the external "boundary" of ER. The action of the secretory complex called Sec complex (which has subunits $\alpha, \beta, \gamma$ ) at the PCC would allow the entrance of both hydrophilic and hydrophobic domains of the molecules entering ER. It has been explained that Sec61 protein would allow translocation of membrane insertion [104] and we consider the mechanism is relevant to the translocation of the EGFR-set into the ER in Figure 9. Now the EGFR-set is inside the ER (i.e. Site (6)). The EGFR-set is translocated eventually from ER to the nucleus (site (7)) via a nucleus pore complex (represented by the slit of the membrane of the cell nucleus). Together with a group of proteins helping to transcribe specific genes, the EGFR-set works at site (8). The anterograde and retrograde transports are well-established evolutionary conserved processes for numerous physiological processes of growth. This retrograde transport has been observed in many cell types [98] and some of the processes have been verified for the pancreatic cancer cells [98] [103] [105]. The fact that the EGFR-set has been found in pancreatic cancer cells supports the notion that the retrograde transport occurs in pancreatic cells also. Microtubules are generated from focal adhesion complexes attached to integrins.

\subsection{Evidence That EGFR Is a Transcription Factor of the Cyclin D1 Gene, Shortening the G1 Phase of Cell Cycle}

Based on the discussion of the last section, we consider that apart from the classical effects of transcription by the STAT dimer (Figure 7) upon activation of EGF, the EGFR-set can be translocated directly from the cell membrane to the nucleus, leading to the transcription of genes responding to the stimulus by EGF. This result provides additional weighting of the influence of EGF on cell growth, differentiation, etc. related to carcinogenesis. On the positive side, as the retrograde transport necessitates the help of proteins such as COPI, dynein motor, dynactin protein, cargo adapter protein, there are more choices of developing inhibitors to stop the retrograde transport of EGF.

Note that long ago, EGFR was already detected in the nuclei of pancreatic cancer cells [106]. It has been pointed out that targeting nuclear EGFR is a crucial step in treating cancer in general in the study of [107]. This important aspect will be followed up in a later section. Note that microtubules form a dense net- 
work in most cells. In $\beta$ cells of the pancreas, they could originate from the Golgi and form a non-radial network. In other cells, they could originate from one side of the cell nucleus and terminate at an integrin-associated protein, which can be taken as part of the focal adhesion [108]. In Figure 9, part of the orientation of the microtubules follow this pattern, and with one terminal connected to a focal adhesion.

EGFR contains a transactivation domain, associating with genes and it can activate sequence-specific gene expression [109]. Using specimens of uterus from pregnant mice, mouse embryos, normal human mouth mucosa, and human cancer tissues labeled EGFR was correlated with highly proliferative activity of cells. EGFR was found to bind and activate AT-rich consensus-sequence-dependent transcription, including the consensus site in cyclin D1 promoter, implying EGFR would be a transcription factor of the cyclin D1 gene, overexpression of which was well established to shorten the G1 phase in cell cycle, leading to proliferation at too fast a speed.

\subsection{On Antibody-Drug Conjugate (ADC) Drugs Targeting Epidermal Growth Factor Receptor (EGFR), Taking a Potential Compound RC68 as a New Example}

Up to the time of writing, four drugs targeting EGFR have been approved by the US FDA to treat other types of cancers (colorectal, esophagus, and lung cancers): 1) cetuximab, 2) panitumumab, 3) nimotuzumab, and 4) necitumumab [110] [111] [112] [113]. We would rather discuss very briefly the working principle of a new class of potential anti-pancreatic-cancer drugs (relevant to targeting EGFR) with one recent example, following the retrograde transport in Figure 9, though the potential drug is not even under clinical trial, because we think that targeting EGF/EGFR is particularly important for a number of cancers, including the pancreatic ones.

An antibody-drug conjugate is a substance made up of a monoclonal antibody chemically linked to a drug that is toxic when the ingredient is released inside the target cell. The monoclonal antibody binds to specific proteins or receptors specific for certain cell type. Drugs with such monoclonal attachment/linkage are called ADCs. Last year, a humanized anti-EGFR monoclonal antibody (RC68) was generated by mouse immunization, and the associated technology is called "complementary-determining region grafting" as described in [114]. The highest level of EGFR expression was revealed (after immunofluorescence staining) on the cells of the human pancreatic cancer cell line BXPC-3, whereas the lowest level of EGFR expression was observed on HEK-293 human embryonic kidney cell line HEK-293 (see (Figure 1a) of [114]).

Immunofluorescence staining also indicated that RC68 was able to bind to 99.9\% of membrane EGFR of BXPC-3 cells. Internalization of RC68-Based ADCs via Endocytosis was observed, indicating that a process such as that shown in Figure 9 was responsible for the internalization. The ADCs showed 
greater cytotoxicity in BXPC-3 cells (up to 70\%) than in another human pancreatic PANC-1 cell line (with cytotoxicity 40\%) (see Figure 5 of [114]), and this difference was interpreted to be due to the difference in EGFR expression level between these two cell lines.

A nude mouse-human pancreatic BXPC-3 xenograft model was used to test the efficacy of this potential drug, with positive result (see Figure 7 of [114]). Note that the BxPC-3 is a human pancreatic cell line for investigating the efficacy of gemcitabine or small molecule tyrosine kinase inhibitors (TKIs), such as erlotinib. This cell line is often used to create the BxPC-3 xenograft mouse model.

\section{The Canonical, Non-Canonical NF-אB Action Pathways and Pathogenesis to Pancreatic Cancinogenesis}

\subsection{The Canonical and Non-Canonical NF-кB Action Pathways}

Members of the Nuclear Factor-kappa B (NF- $\kappa \mathrm{B})$ family are transcription factors that regulate the expression of a wide range of genes for inflammatory and immunity responses, cell survival, and cell proliferation. Referring to Figure 10, in the first step, when a cytokine receptor registers a ligand (cytokines like tumor necrosis factor- $\alpha$ (TNF- $\alpha$ ), interleukin), a signal is sent to a protein complex (called IKB kinase, or IKK) which consists of two kinases (IKKa and IKK $\beta$ ) and a regulatory subunit named $\mathrm{NF}-\kappa \mathrm{B}$ essential modulator (NEMO, or IKK $\gamma$ ). Now the NF- $\kappa B$ transcription factor family in mammals consists of five proteins, 1) NF- $\kappa \mathrm{B} 1$ (also called p50), 2) NF- $\kappa \mathrm{B} 2$ (also called p52 and its precursor is p100), 3) RelA (alias p65, v-rel reticuloendotheliosis viral oncogene homolog $\mathrm{A}$ (avian)), 4) RelB, 5) c-Rel. These five members associate with each other to form distinct transcriptionally active homo- and heterodimeric complexes. The "canonical NF- $\kappa$ B members" predominantly form dimers of (p50/RelA) and (p50/c-Rel) [115] [116]. In the dormant state, the dimer (take, for example, a combination of NF- $\kappa \mathrm{B} 1$ and RelA), binds to the inhibitor I $\kappa \mathrm{B} \alpha$. The activated IKK phosphorylates $\mathrm{I} \kappa \mathrm{B} \alpha$, which is then degraded in the proteasome. Concurrently, the dimer (NF- $\kappa \mathrm{B} 1$ and RelA) is translocated to the nucleus and binds to the response element of a specific sequence of the DNA, leading to transcription of the gene into mRNAs. The corresponding protein is then synthesized in the usual way.

For the non-canonical NF- $\kappa$ B pathway, proteins Traf2, Traf3 are attached to the non-canonical receptor at the cell membrane. These two proteins, together with some other supporting proteins form a complex; this complex causes continual degradation of the NF- $\mathrm{BB}$-inducing kinase (NIK), whose activity triggers off the non-canonical pathway. There are several receptors of the non-canonical pathway. We just take one example here. The Lymphotoxin $\beta$ receptor (LT $\beta R$ ), is a cell surface receptor for lymphotoxin involved in apoptosis and cytokine release [117]. Here Lymphotoxin is a member of the cytokines, whose members are responsible for regulating the function of and cell growth, and are expressed 
by a wide variety of cells [118]. When receptor LT $\beta R$ is docked by the ligand Lymphotoxin, via the action of the Traf2-Traf3 complex, NIK is not degraded and NIK phosphorylates the dimer IKK $\alpha$, as indicated schematically on the right side of Figure 10. IKKa, in turn, phosphorylates the transcription factor dimer formed by NF-kB plus RelB (precursor of NF-kB2(p100)). The dimer (RelB and $\mathrm{NF}-\mathrm{\kappa B} 2(\mathrm{p} 52)$ ) is translocated to the nucleus. As the two NFkB pathways occur in most cells [116], we show the typical products of proteins synthesized in the middle of Figure 10 according to references [116] [119].

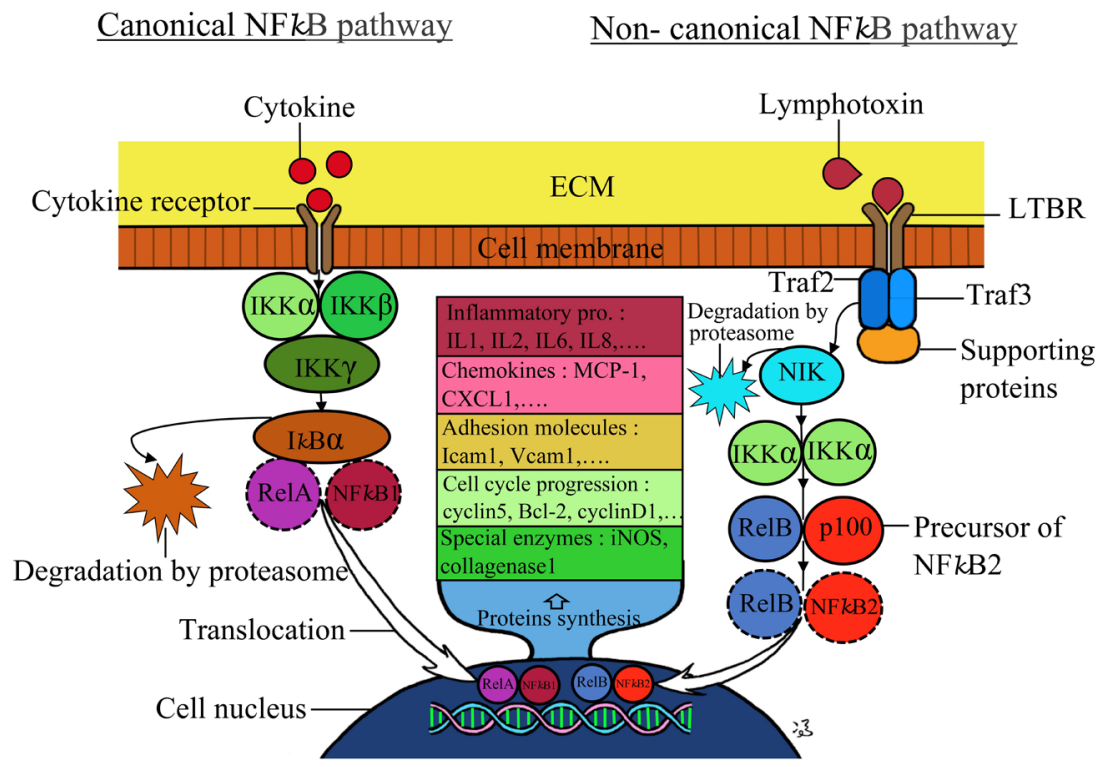

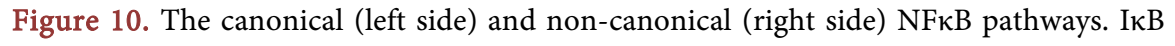
kinase $\alpha, \beta, \gamma=\mathrm{IKK} \alpha, \mathrm{IKK} \beta, \mathrm{IKK} \gamma$ respectively. Pro. = proteins. IL = interleukin, $\mathrm{MCP}-1$ $=$ monocyte chemoattractant protein 1, CXCL1 = chemokine C-X-C motif ligand-1, Icam $1=($ Inter $)$ cellular adhesion protein $1 . \mathrm{Vcam} 1=$ vascular cell adhesion protein 1. cyclin $\mathrm{D} 1=$ a protein that helps control cell division. Bcl-2 = B-cell lymphoma 2 . iNOS = inducible nitric oxide synthase. Collagenase 1 degrades collagens of certain types. $І \kappa \mathrm{B} \alpha=$ inhibitory $\kappa$ protein type $\alpha$. NFאB1, $2=\mathrm{NF}-\kappa \mathrm{B}$ transcription factor type1, 2 respectively. $\mathrm{NIK}=\mathrm{NF}-\kappa \mathrm{B}$-inducing kinase. LT- $\beta \mathrm{R}=$ Lymphotoxin receptor type $\beta$. RelA $=$ v-rel reticuloendotheliosis viral oncogene homolog A (avian), is also called p65. RelB = v-rel reticuloendotheliosis viral oncogene homolog $B$ (avian). NFkB1,2 are two members of the $\mathrm{NF}-\kappa \mathrm{B}$ transcription factor family. p100 is the precursor of NF- $\kappa \mathrm{B} 2$. The cytokines include interleukins, TNF- $\alpha$. For other details, see text. This figure was painted by author PCWF.

\subsection{Proteins Relating to Inflammation, Cell Adhesion, Cell Cycle Progression, Collagen Remodeling and Immunity Are Synthesized in Response to Activation of NF- $\mathrm{KB}$}

Many proteins can be synthesized by these canonical or non-canonical pathways, even with one type of stimulation. For example, for inflammatory response, interleukins IL-1 $\alpha$, IL2, IL6, IL8, Tumour Necrosis Factor $\alpha$ (TNFa, an inflammatory cytokine produced by macrophages/monocytes), cyclooxygenase-2 (COX-2), an enzyme involved in the process of inflammation, can be 
generated [116].

The chemokines monocyte chemoattractant protein 1 (MCP-1), chemokine $\mathrm{C}-\mathrm{X}-\mathrm{C}$ motif-ligand 1 (CXCL1) are produced for recruiting monocyte, neutrophil and other immunity cells during angiogenesis and inflammation. Adhesion molecules such as ICAM1(Intercellular adhesion protein 1) [120], vascular cell adhesion protein 1 (VCAM1) [121], which regulate the vascular permeability, allowing leukocytes transmigration to the ECM for inflammatory action, are also synthesized. There are also proteins related to stress response, such as angiotensin II, a vessel constrictor. Anti-apoptotic factors, such as the B-cell lymphoma 2 (Bcl-2), which is a key member of regulator proteins that regulate apoptosis, is another stress protein. Cyclin D1(a regulatory subunit of cyclin-dependent kinases CDK4 and CDK6) is a protein that helps control cell division (see Section (V) on CDNK2A). Transforming growth factor- $\alpha$ is a member of the epidermal growth factor (EGF) family, being a mitogenic polypeptide; it belongs to the group of cell cycle regulation [122]. The Systemic enzyme inducible nitric oxide synthase iNOS (one of the three isoforms of nitric oxide) produces a large amount of nitric oxide during immunity response. Nitric oxide combines with ROS superoxide to become peroxynitrite (ONOO) ${ }^{-}$which is detrimental to cell death (of the health cells). Collagenase1 digests collagen during ECM remodeling. All these proteins/peptides (as listed in the middle part of Figure 10, can be generated via the two NF- $\kappa$ B pathways [116].

\subsection{Involvement of the NF-кB Pathway in the Pathogenesis of Pancreatitis, Angiogenesis, and Pancreatic Ductal Cancer}

Using peripheral blood mononuclear cells isolated from patients' blood, it has been found by measurement [123] that Both NF- $\mathrm{KB}$ and heat shock factor-1(HSF-1) would be elevated systemically in patients suffering from acute pancreatitis. Based on the finding that HSF-1 is inversely correlated with the Acute Physiology Score (APS) and Acute Physiologic Assessment and Chronic Health Evaluation II (APACHE II) score, it has been inferred that HSF-1 activation could protect the organ against the severity of pancreatitis. In fact, the mechanism of the protective effects of heat shock proteins for general cancer has been analyzed in our previous work, and we agree with this proposal; for details, see [3].

Using genetically engineered mouse models, it has been demonstrated [124] that a pathway linking dual forward loops of IL-1a /p62 through which IKK $\beta / \mathrm{NF}-\kappa \mathrm{B}$ is activated by $\mathrm{Kras}^{\mathrm{G} 12 \mathrm{D}}$ [125]. Here p62 (SQSTM1) is a multifunctional stress-inducible scaffold protein involved in diverse cellular processes. Noting that mutational activation of KRAS has been detected in $80 \%-95 \%$ of PDAC by the year 2000 [126] and activation of NF- $\kappa B$ in the epithelial cells of the pancreas leads to the synthesis of IL-1a (also called hematopoietin 1) and other inflammatory cytokines, together with antiapoptotic proteins; such result implies that pancreatitis and then pancreatic carcinogenesis could be sustained, once initiated. 
In 2009, two pancreatic cancer cell lines BxPC-3 and SW 1990, human umbilical vein endothelial cells (HUVECs) were used, and cell invasion, angiogenesis assays were applied to study pancreatic EMT [127]. It was found that IL-1a mRNA levels in the cancer cells were significantly higher than the control. Vessel tubes formation and migration of the HUVECs were observed when co-cultivated with the cancer cells.

In another investigation, the human pancreatic cell lines PANC-1, BxPC were employed. It was demonstrated that with overexpression of the hypoxia-inducible factor-1 $\alpha$ (HIF-1 $\alpha$ ) (Figure 2 and Section (III.2)), the cells underwent EMT in an NF- $\kappa \mathrm{B}-$ dependent manner [128]. It was also noted that inhibition of NF- $\kappa \mathrm{B}$ activity would reverse the EMT phenotype. Putting the results reviewed in Sections (9.1-9.3) above suggests that there are interactions between the NF- $\kappa$ B pathways and the HIF-1a-VEGF pathway (in Figure 2), leading to angiogenesis and concurrent EMT progression towards carcinogenesis, with NF- $\kappa$ B playing a significant role. Looking for inhibitors of NF- $\kappa \mathrm{B}$ is desirable.

\subsection{Searching for Inhibitors of HIF-1 $\alpha$ and NF-кB-APX3330, SLC-0111, Agonist of IL-10}

So far, 16 carbonic anhydrases (Cas), which are expressed in human tissues, interact with HIF-1 [129] [130]. Among these 16 anhydrases, CA9 has been shown to be driven by HIF-1 activity. On the other hand, HIF-1 transcriptional activity is enhanced by Apurinic/Apyrimidinic Endonuclease-1-Reduction/oxidation Effector Factor 1(APE1/Ref-1) [131]. The small molecule APX3330 is a potent inhibitor of APE1/Ref-1. This potential drug is about to proceed to Phase II trial. APX3330 has been found to regulate activity not only of HIF-1 $\alpha$, but also NF- $\kappa$ B and Jak-STAT3 (see Figure 7) [132]. Another molecule called SLC-0111, which inhibits the action of CA9 at the cell membrane, is under preclinical experimentation [133].

More than 2 decades ago, a synthetic interleukin-10 agonist IT 9302 was found to reduce the mortality rate (from $60 \%$ to $0 \%$ ) in rabbit models of acute necrotizing pancreatitis. It was suggested that the mechanism of action could be due to inhibition of circulating levels of TNF- $\alpha$, IL8, which could be generated via the NF- $\kappa$ B pathway just discussed [134].

\section{The Hedgehog Pathway and Its Involvement in Human Primary Pancreatic Adenocarcinomas}

\subsection{The Hedgehog Pathway and the Proteins Synthesized upon Activation}

The Hedgehog signaling pathway is a crucial regulator of proliferation and differentiation during embryonic development. The two key transmembrane proteins are: 1) Protein patched homolog 1 (Patch 1) is a member of the Patch protein family; so far two members are found, and Patch 1 is relevant to our discussion. 2) Smoothened protein (Smo). In the quiescent state Patch 1 inhibits the action of Smo. The suppressor of fused homolog (Sufu) forms a complex with 
the Glil protein in the cytosol. The Glil protein contains five successive repeats of a zinc finger which can bind to DNA for transcription [135]. There are five members in the Gli family. The quiescent state is represented schematically by the left side of Figure 11.

We note that three hedgehog ligands have been identified-Sonic hedgehog (Shh), Indian hedgehog (Ihh), and Desert hedgehog (Dhh); these ligands could send signals to receiving cells at a distance greater than cell sizes involved [136]. When a hedgehog ligand (such as Shh) binds to a domain receptor of the Patch protein, the inhibition of the Patch protein on Smo is relieved. The activated Smo binds with Sufu, which then releases the transcriptional factor Zinc finger protein (Gli1), followed by translocation of Gli1 to the cell nucleus, inducing transcription of the following proteins [137]: 1) myc, a proto-oncogene which encodes a nuclear phosphoprotein that plays a role in cell cycle progression, apoptosis and cellular transformation; 2) cyc D1, a cyclin that functions as a regulator of a cyclin-dependent kinase (CDK) involved in regulating transcription, mRNA processing, and the differentiation of nerve cells; 3) Patch 1, to replace the degraded one after enzymatic action; 4) Bcl-2 (or B-cell lymphoma 2) is the key member of the $\mathrm{Bcl}$ family of regulator proteins that regulate apoptosis; 5) Sox2 (or sex determining region Y-box 2), is a transcription factor essential for maintaining self-renewal stem cells. We thus see that dysregulation of the hedgehog pathway may readily lead to the formation of cancer cells, based on the functions of the proteins produced in response to the activation of a receptor of a hedgehog ligand.

(a)

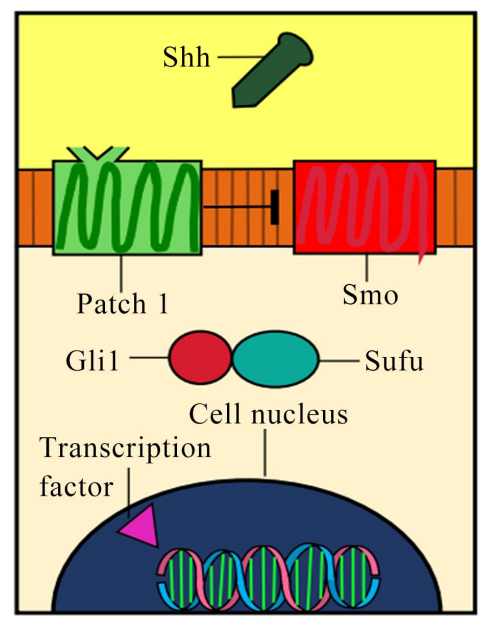

No transcription (b)

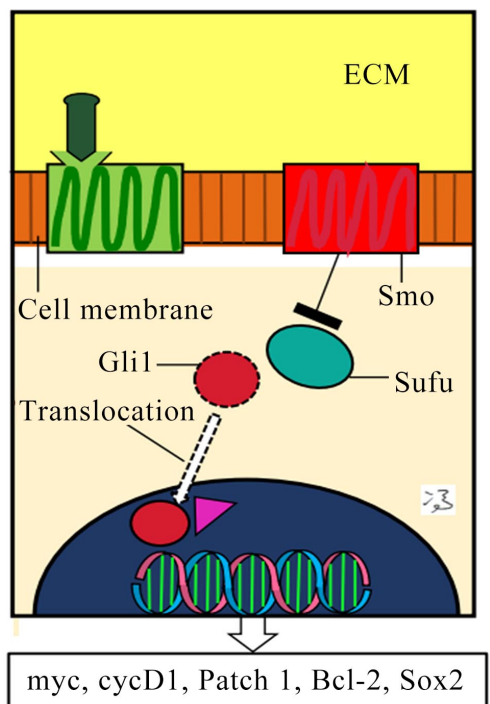

Figure 11. Sufu locks to Gli1 in the quiescent state. When a Hedgehog ligand, such as Shh binds to Patch1, the transmembrane Smo is activated, which then binds to Sufu, releasing the transcriptional factor to the nucleus, leading to transcription of genes (then synthesis of the proteins) as marked at the lower right corner. For other details, see text. This figure was painted by author PCWF. 


\subsection{Evidence of the Involvement of the Hedgehog Pathway in Progression to Human Primary Pancreatic Adenocarcinomas}

It has been reported that the hedgehog pathway can be aberrantly activated in many solid tumors, such as skin cancer [138], prostate cancer [139], medulloblastoma [140], colorectal cancer [141], ovarian cancer [142], pancreatic cancers [143]. A number of inhibitors of the active proteins of the hedgehog pathway have been attempted in in vitro and some clinical trials with some success-showing signs of efficacy; the results, in general, are not promising (see e.g. the negative result of using Vismodegib to treat colorectal cancers according to the study of [141]). Since the environment of different cancers can be significantly different, despite the controversial results; even with one positive result, it is worth investigating the mechanism of the successful case.

In the study of [136], the expressions of protein members involved in the hedgehog pathway (particularly the ligand sonic hedgehog (Shh) and receptor Smoothened (Smo)) were analyzed in 1) pancreatic cancer and 2) pancreatitis specimens. Technically, the human pancreatic Nestin-expressing cell line (HPNE) was used as a control cell line; these cells were derived from normal pancreatic tissue and immortalized (with telomerase) using hTERT (a catalytic subunit of the enzyme telomerase, which, together with the telomerase RNA component (TERC), comprises the most important unit of the telomerase complex. The technicality of preparation is described in [144]). Two more human immortalized control fibroblasts cultures (SC2 and SC3) were also established from non-malignant pancreatic tissues. SC2 was derived from an intraductal papillary mucinous neoplasm (IPMN) of the pancreas, and SC3 was derived from resected chronic pancreatitis tissue. Gene expression profiling analyses of 1) the human pancreatic cancer-associated fibroblasts (CAFs or named stellate cells in this paper) and 2) non-neoplastic pancreatic fibroblasts were carried out. Total RNA was isolated from 1) and 2) cell cultures using a commercial miRNA kit.

The expression of the SMO protein was examined by immunohistochemical labeling of eight tissue microarrays containing a total of 156 different surgically resected pancreatic ductal adenocarcinoma. IPMNs, and chronic pancreatitis tissues (i.e. SC3) were constructed according to the method of [145].

Analyzing the stated specimens, the findings are: 1) SMO is upregulated in human pancreatic activated stellate cells. 2) Note that Zinc finger protein Gli1 is a transcriptional activator and is found to mediate Shh signaling [135]. The Shh ligand has been found to induce expression of Gli1 mRNA in pancreatic stellate cells. 3) siRNA knockdown of SMO expression blocks GLI1 induction in pancreatic stellate cells. 4) Hedgehog pathway has been confirmed active in pancreatic cancer associated stromal cells in vivo using mouse xenografts [136]. 


\subsection{Combination of Hedgehog Inhibitor (Cyclopamine) and Chemotherapy Drug Paclitaxel Delivered by Nanoparticles Demonstrated Efficacy in Treating Patient-Derived Xenograft Model}

One major hurdle to clinically treat PDAC, as compared with other solid cancers, is the existence of an extensive desmoplastic stroma, which is a fibrotic deposition produced by stellate cells in the organ [146]. In the study of [147], in order to overcome the stated hurdle, a sonic hedgehog inhibitor, cyclopamine (CPA), and a cytotoxic chemotherapy drug paclitaxel (PTX) with a polymeric micelle formulation (called M-CPA/PTX) was prepared as a drug to treat cancer models. CPA is supposed to deplete the stroma-producing stellate cells (or cancer-associated fibroblasts (CAFs)), while PTX has been known to have efficacy to inhibit tumor proliferation (acinar cells). The stellate cells have been tested to be $\alpha$-SMA positive (see a later section to describe the stellate cells).

Three (1 - 3) orthotopic xenograft models were used in this investigation on efficacy and related mechanism: 1) Human pancreatic cancer cells MiaPaca-2 were injected into the pancreases of the mouse models to prepare the orthotopic xenografts. 2) Patient-derived xenograft model, which is similar to 1) but preserving many features of tumor heterogeneity and introducing human leukocyte populations [148]. 3) The KPC models, where alteration of the genes KRAS, TP53, Cre are genetically engineered; here Cre is a special tool gene that is used to control where KRAS and TP53 are turned on (see details of the mechanism in [149]). In the three groups 1) - 3) stated above in the investigation of [147], different combinations of treatment settings were adopted: a) untreated (control); treated with b) M-CPA; c) M-PTX; d) M-CPA/PTX. M-CPA/PTX nanoparticles were delivered to the tumor site by blood circulation. The size of the nanoparticles is $<150 \mathrm{~nm}$. Due to the natural leakiness of the intratumoral vasculature, and based on the analysis of this issue reported in ref. [150], there was a high chance of M-CPA/PTX being extravasated into the interstitial fluid of the ECM. It is anticipated that both CPA and PTX were released to affect the behavior of the stellate and tumor cells. Since the tumor sizes of the models strank back about to normal after such treatment (Figure 6 of [147]), the combined effects of M-CPA/PTX must have worked to eliminate the proliferating cells. There was evidence that the micro-vessel density was increased, hypoxia was alleviated, matrix stiffness was reduced; there was indirect evidence that the tumor-restraining function of the extracellular matrix was maintained. Based on the variation of the protein expression of the $\alpha$-SMA of the stellate cells, there was indication that the vicious cycle of mechanotransduction interaction between the stellate and tumor cells was disrupted (detail will be described in a later section). A model of the efficacy is presented in Figure 7 of [147]. We wish to point out that the activation of at least some stellate cells seems to be closely related to the sonic hedgehog pathway; other growth factors/stimulations can activate a stellate cell also. 


\section{The Importance of Stopping Tumorigenesis at the Early Stage by Inhibiting the Notch Signaling Pathway}

\subsection{The Notch Signaling Pathway between Adjacent Cells and EMT, Tumorigenesis}

The Notch signaling pathway is a highly conserved cell signaling pathway, restricted to neighboring cells in all Mammals [151] [152]. Notch has four different notch receptors (each is a single-pass transmembrane protein): Notch1, Notch2, Notch3, Notch4, [153]. Notch family members control cell fate decisions during development; for that reason, the signal of proliferation can be transmitted to adjacent cells. There are five functional ligands, JAG1, JAG2, DLL1, DLL3, and DLL4 discovered so far known. Notch signaling has also been found to be dysregulated in some cancers, implying such signaling is important in developing anti-cancer target therapy (see e.g. [154]).

In Figure 12, we label the Ligand and receptor as Jag and Notch respectively as an example (leaving out the member number). Both the receptor and ligand have their main physical parts in the extracellular domain of the adjacent cells. Up to now, we only know that the receptors tend to be cell-shape dependent (preferably located at the tip) for the ligand-receptor docking in some animals [155]. We can only start from the pathway after the docking process. Stimulated by a signal from the ligand in the signal sending cell (cell 1), two proteolytic enzymes are recruited in the internal side of the membrane of signal receiving cell (cell 2): 1) ADAM10/TACE (of the metalloprotease family); 2) $\gamma$-secretase. The bonding causes a conformational change in the extracellular juxtamembrane region of the receptor Notch, rendering Notch to be susceptible to successive cleavages by ADAM metalloproteases and $\gamma$-secretase (see Figure 1 of [151]). It has also been inferred that the triggering force is mechanical, arising from ligand endocytosis [156].

After the stated cleavage processes, the intracellular domain of the Notch receptor (called Nicd) is translocated to the nucleus. There the DNA binding protein/(transcriptional regulator) called CSL (or CBF-1) and co-repressor CoR binds to the DNA, keeping DNA in a quiescent state. Nicd enters the nucleus and activates CSL, recruiting the co-activator (CoA), mastermind-like 1 (MAML1) plus others to form a transcriptional activator complex. This complex causes transcription of certain genes, leading to the synthesis of the corresponding proteins. Relevant to pancreatic cancer, they are [157]: 1) C-myc, a proto-oncogene and encodes a nuclear phosphoprotein that plays a role in cell cycle progression, apoptosis, and cellular transformation. This gene is pleiotropic, but the amplification of this gene is commonly detected in numerous human cancers. 2) cyclin D1, a cyclin functions as a regulator of a Cyclin-dependent kinase (CDK) which is involved in regulating transcription, mRNA processing, and the differentiation of nerve cells. Cyclin D1 forms a complex with CDK4 or CDK6, whose activity is required for cell cycle G1/S transition (see Figure 4). Overexpression of cyclin D1 is found in many cancers. 3) Transcription factor Hes1 (hairy and en- 
hancer of split-1) is encoded by the gene bearing the same name. There are 7 members in the family (Hes1-7). HES genes code nuclear proteins that suppress transcription [158]. 4) The Hairy/enhancer-of-split related with YRPW motif protein 1 (or in short, Hey1) is a transcriptional repressor protein [159]. 5) Cyclooxygenase 2 ( $\mathrm{COX} 2$ ), is an enzyme responsible for the synthesis of prostaglandins which promote inflammation, pain, and fever. 6) Vascular endothelial growth factor (VEGF), as a protein, participates in the growth of (blood) vessels (see Figure 2). 7) The enzymes matrix metalloproteinases (MMPs) are involved in the breakdown of collagen in the extracellular matrix, during embryonic development, reproduction, angiogenesis, bone development, wound healing, cell migration [2]. The MMPs are necessary for numerous physiological processes. MMP 9 is more specific to the degradation of the basal membrane, relevant to the invasion of cancer cells. Looking down the list 1)-7), the proteins resulting from the Notch signaling is involved in daily physiological processes, including wound healing. However, from the high correlation of over-expression of Notch and the occurrence of tumors, obviously, the quantities of these proteins are not balanced during EMT, to tumorigenesis.

Going back to the Notch signaling process. The Ligand left in cell 1 actually participates in another set of processes, sending a signal back to the nucleus of the signal-sending cell. To simplify matters, we leave the readers to the description of this part of the Notch process to [160].

\subsection{The Non-Canonical Notch Pathway and Mirco-Tumorigenesis by Stem Cells; Inhibitors of the Notch Pathway as a Potential Drug $-\gamma$ Secretase Inhibitor R04929097 Plus Gemcitabine Was under Phase II Trial by 2014}

In general, when the signaling process is limited to adjacent cells, it is called canonical. Using actin-based cellular projections, which include the Notch ligands, to deliver activating signals to a Notch process at distant sites is called non-canonical, as demonstrated with the Drosophila model [161]. Malfunctional or dysregulated Notch signal is related to abnormal growth and can be associated to the development of micro-tumorigenesis, in our view.

This cell-cell communication is inferred to occur during the development of the EMT and tumorigenesis states of the acinar cells, which connect to form a ring shape structure and the "outlet" of the ring simply joins the duct cells of the pancreatic duct.

Tissues of primary human pancreatic tumors of 8 patients were used to prepare the (mice) xenografts in the study of [162]. Triple-marker profile CD44+/ CD24+/ESA+ (ESA is an epithelial surface antigen) was used to isolate cancer stem cells from cancer cells from the xenograft tissues [163]. Some expression levels of Notch signaling pathway components in cancer stem cells were compared to that of the bulk tumor cells. The gamma secretase inhibitor R04929097, plus gemcitabine were found to have anti-cancer effects to suppress the degree of 
carcinogenesis. Note that such a combination was taken to phase I trial for the treatment of advanced solid tumors excluding pancreatic cancer in 2012 [164]. The expression levels of three proteins/RNA, i.e. Hes1, Nicd (detected by cleaved Notch), and actin were used as markers since their expressions were more than 1.5 folds compared to the primary tumor. shRNA Hes1 was applied to confirm the result.

In the resectable tumorigenic cases, the high occurrence frequency of distant relapse following successful surgery suggests the spread of cancer cells before surgery. Identification of the activation of the Notch pathway in the pancreatic cancer stem cells, it is thus plausible to treat patients with drugs targeting Notch right at the beginning of the discovery of the disease. Note that the gamma secretase inhibitor R04929097 was taken as the drug for phase II trial in patients previously treated metastatic pancreatic adenocarcinoma by 2014 [165].

(a)

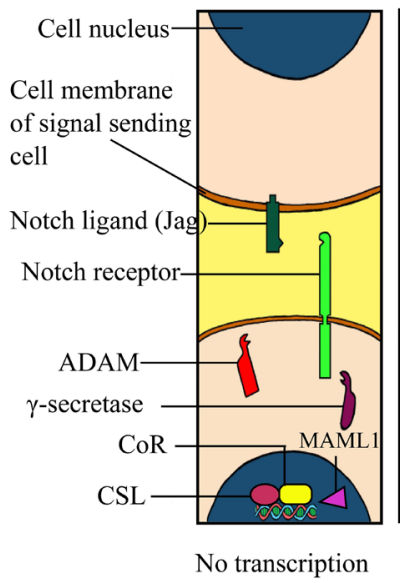

(b)

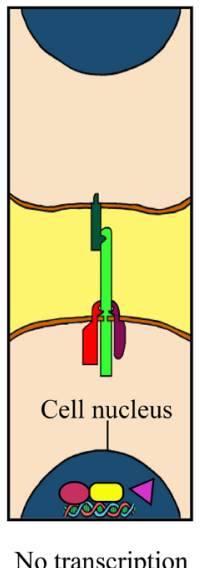

(c)

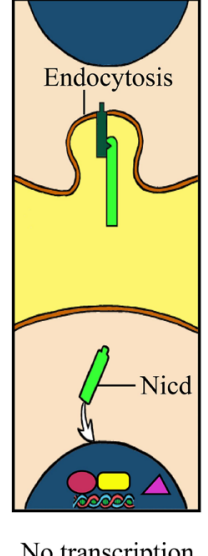

3

(d)

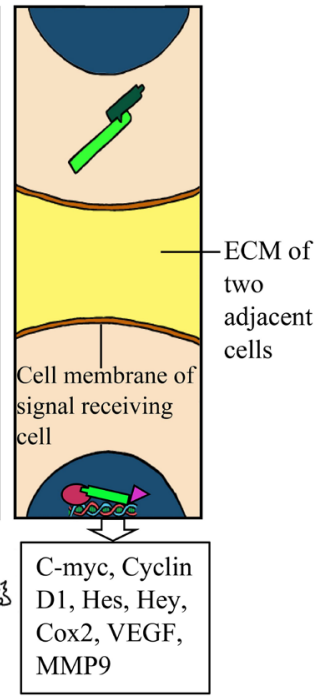

Figure 12. The canonical Notch pathway is restricted to neighboring cells, with parts of the nuclei shown in this figure. Jagged1 (encoded by JAG1) is one of the five cell surface proteins (ligands) that interact with 4 Notch receptors in the mammals. The DNA binding protein called CSL (light red) and co-repressor CoR (yellow) binds to the DNA, keeping it in a quiescent state. The mastermind-like 1 (MAML1, in purple) is also in the dormant state. There is no proliferation resulting in this pathway in (a)-(c). Here Jag1 was painted in deep green and Notch1 in light green. The two cleavage enzymes ADAM (red) and $\gamma$-secretase (brown) are inactive when the pathway is not turned on. When Jag1 binds Notch1, signals are sent to the stated enzymes and they begin to cleave the Notch1 receptor into two parts. The intracellular domain of the Notch receptor (called Nicd, light green) is translocated to the nucleus, binding CSL and MAML1. While Jag1 with the extracellular part of Notch1 protein is engulfed by endocytosis in the signal sending cell, transcriptions of C-myc, cyclin D1, Hes, Hey, Cox2, VEGF, and MMP9 are carried out in the nucleus of the receiving cell. See text for the functions of these 7 proteins. The number 1 has been omitted in the key members for simplicity, as other members give similar results in different cells. The extracellular matrix of these two adjacent cells is represented by a yellow background, which contains many constituents as described in Figure 2 of [166]. 


\section{One Major Factor of the Progression of Pancreatic Ductal Cancer: Transformation of the Quiescent Stellate Cells to the Myofibroblast Type Cells}

\subsection{A Quiescent Stellate Cell Has Numerous ATR Drops in the Cytoplasm}

Pancreatic stellate cells (PSCs), amounting to $4 \%-7 \%$ of the mass of the gland [11], are found to be adjacent to the basolateral aspects of pancreatic acinar cells and around pancreatic ducts and blood vessels [12]. PSCs have been detected around islets too [167].

Based on staining examination for the marker desmin (encoded by DES), which is a cytoskeletal protein, a quiescent PSC appears as an elongated cell, with a central cell body, extending along the base of adjacent acinar cells. Quiescent PSCs have the capability to store retinoids (with their analogs) inside fluid droplets [168]. Retinoid and its metabolites have been shown to inhibit the expression of $\alpha$-SMA and to decrease activation of relevant signaling pathways involving invasion and cross-talks with the acinar cells [169].

The quiescent cells proliferate at very slow rates and migrate only within the parenchymal tissues of the pancreas. However, when they lose their lipid droplets and begin to show a-SMA tubule structures, they begin to transit to the myofibroblast-like phenotype [170], in response to stimuli like growth factors, inflammatory signals, to be specified in a later section. In Figure 3, the proteins forming the focal adhesion painted are Talin, Fak, Paxillin, Zyxin, YASP, $\alpha$-actinin, and "attachment protein" Vinculin. In a quiescent stellate cell, the focal adhesion is not formed. In Figure 13 only three separate proteins such as

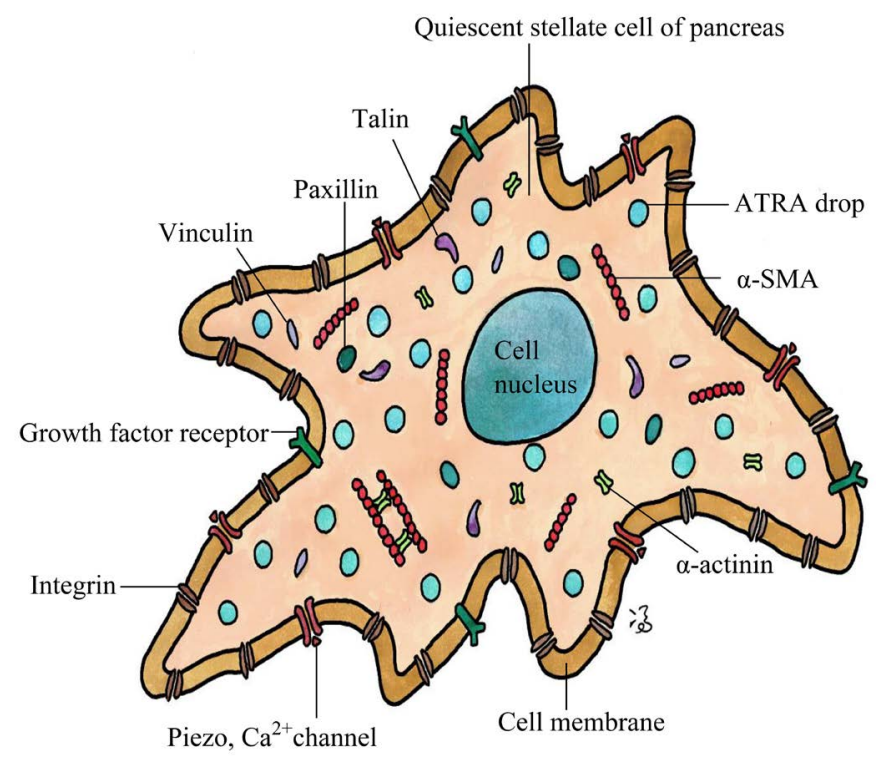

Figure 13. The quiescent state of a stellate cell of the pancreas. There are many ATRA (oil) drops (blue) in the cytoplasm. Paxillin (green), talin (purple), vinculin (light purple) represent some of the proteins forming the focal adhesion and joining (via vinculin) the actin chains, which are built of $\alpha$-SMA and $\alpha$-actinin. The green $\mathrm{Y}$-shape proteins are growth factor receptors. This figure was hand-painted by author PCWF. 
Talin (purple), Paxillin (green), Vinculin (light purple) in the cytoplasm have been painted to represent such individuals of proteins. The actin chains are formed by a-actin (red small circles), and "glued" by a-actinin (light green), but the chains are not formed yet without the action of Vinculin. The light blue circles are ATRA drops that have been clearly revealed in experiments in the stellate cells (see Figure 2 of [168]).

Integrins (brown), each built of one $\alpha$ unit and one $\beta$ unit, are transmembrane proteins. Without mechanical (or some chemical) stimulation, they do not act in any manner. The green $\mathrm{Y}$-shape structure represents a growth factor receptor (such as TGF, EGF). There are calcium ion channels Piezo (mainly class1) in the cell membrane too. A "stopper" closes the entrance of extracellular calcium in each one without mechanical traction from the integrin/collagen fibrils of the basal membrane.

\subsection{Activation Mechanism of a Stellate Cell by Transforming Growth Factor TGF $\beta$ and the Occurrence of Fibrosis around the Activated Stellate Cell}

When a pancreatic stellate cell is deprived of vitamin A droplet, a stellate cell can readily be activated. In this section we consider one triggering mechanism to be TGF $\beta$ activation as an example.

We first note that in the quiescent state the TGF $\beta$ (bright green) binds to the protein LAP (deep blue) and LTBP-1(black), forming a complex (sites (1), (2) of Figure 14). LTBP-1 has a strong affinity to fibrillin (orange), a glycoprotein in the ECM, particularly associated with elastin (see Section (1) of [2]). Using lung fibroblasts and vascular smooth muscle cells, it has been found that one end of LTBP-1 protein is attached to fibrillin in the ECM [171]. The other end of LTBP-1 has an affinity to fibronectin fibril (light purple), also a glycoprotein, which is readily attached to unit $\beta$ (the slightly longer unit of the integrin, whereas the other unit is called $\alpha$ ) [171]. The contraction of the cell membrane of a stellate cell would have a larger chance of attracting the LTBP-1 complex. However, at the normal state, the TGF $\beta$-LAP-LTBP- 1 complex stays dormant.

Many cytokines would cause the integrin to contract, and TGF $\beta$ itself is one of them. A pull from collagen fibers at a distance can also transmit a force to the integrin, and the reaction is the formation of a focal adhesion complex (as represented by the blue ellipsoid here) by the connection of paxillin, talin, and other proteins (already described in detail in Section 9.1 with Figure 3). The focal adhesion complex also causes the $\alpha$-SMA fibrils (small red circles) to be lined up, with proteins such as $\alpha$-actinin (green clip-like structure) as "glue". Since the $\alpha$-SMA fibrils can contract as muscles do, the morphology of the cell can be changed so that the cell can crawl along the collagen fibrils/fibers (yellow) in the ECM according to the rules of durotaxis and chemotaxis (see [2] [172]). As the cell starts to exert a contract force on the ECM, the cell membrane contract via the action of the integrins. At site (3), a TGF $\beta$-LAP-LTBP- 1 complex is attracted to an integrin. Further contraction of the cell membrane would "pull" 
the LAP protein, releasing TGF $\beta$ as shown in the site (4). On the other hand, the contraction force of integrins can also open the newly discovered calcium ion channel Piezo1 (deep red). The entry of $\mathrm{Ca}^{2+}$ from an extracellular source (indicated by the dotted arrow on the right side of the site (5)), would trigger off the Rho-Rock pathway leading to the lining up of more $\alpha$-SMA fibrils, shown in Figure 13 and Figure 3. Both the contraction of the integrin and entry of $\mathrm{Ca}^{2+}$ would lead to the formation of focal adhesion.

Now the free TGF $\beta$ has an affinity to its own receptor TGF $\beta$, as in site (6). The receptor of TGF $\beta$ is in the active state, initiating more activity of the Rho-Rock signaling pathway. Some a-SMA fibrils line up to join the nucleus, initiating the transcription (and hence protein synthesis) of the digestive enzyme MMPs (plus also their regulators, metalloproteinases inhibitor (TIMPs)), represented by the fan-like structure painted in red on one side and white on the other). This is a natural response because the enzymes must be secreted to the ECM to digest the collagen fibrils to leave room for the cell to migrate. The cell is now a myofibroblast, with the intention to do wound healing work. Therefore, procollagen (triple helix in the upper part of the cell (for details see [2]) must be synthesized also. These procollagen molecules are also secreted to form collagen molecules proper and then collagen fibrils. When there are more collagen fibrils/fibers in the ECM, the mechanical tension on the cell membrane is automatically greater. The integrins sense such tension, and the $\alpha$-SMA reacts more strongly than before. We see that a slight imbalance of the above complicated processes would lead to a vicious cycle, with more accumulation of collagen and a stronger contraction of the stellate cells. The activated stellate cells are communicating with the acinar cells, and $\beta$ cells of the islets and an epithelial-mesenchymal-transition (EMT) has occurred.

Using lung fibroblasts, and vascular smooth muscle cells, it has been found that LTBP-1 protein is attached to fibrillins in the ECM, whereas to release TGF- $\beta$ from the latent complex, the LTBP-1 needs to bind to fibronectin also. Note that fibrillin is a glycoprotein being attached to microfibrils associated with elastin in the ECM around the pancreas. Fibronectin is well established as a glycoprotein also, and it has a high affinity to integrins. Although the cells used in the experiments of [171] were not pancreas cells, yet the TGF activation is an evolution conserved process, and we take the above statement as the model to describe the release of TGF in the ECM of pancreatic cells. The stated connection of LTBP-1 is supported by research of other groups (see e.g. [173]).

When a PSC changes from the quiescent to the activated myofibroblast phenotype, the following events occur: 1) loss of ATRA lipid droplets; 2) excessive production of collagen; 3) excessive production of proteoglycans and glycol-proteins-in particular, hyaluronan which is electrically polarised to attract water to form a large volume of think interstitial fluid [37] [166]; 4) overproduction of inhibitors of matrix metalloproteinases (TIMPs) so that the accumulated collagen is not digested; 5) overexpression of HSP47 in PSC. Note that in the 
event 1) ATRA keeps PSC quiescent by regulating the target genes by binding to the associated nuclear receptor (for details see [174]). There is evidence that overexpression of HSP47, which is specific in folding the native collagen in the ER [3], is implied in PDAC fibrosis [175].

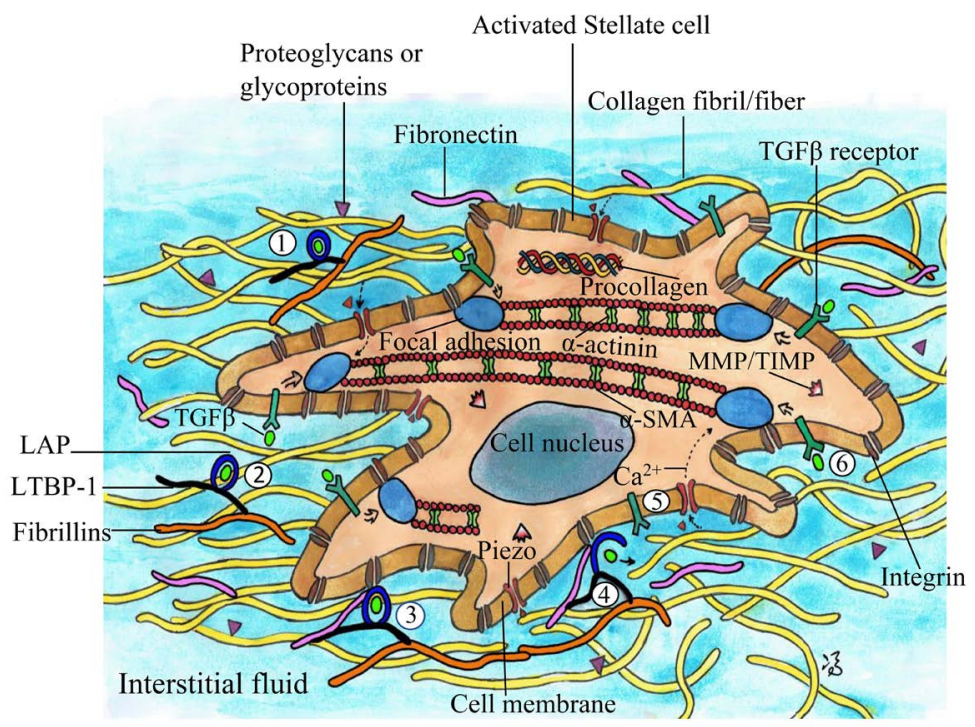

Figure 14. The transforming growth factor TGF- $\beta$ (bright green) is enclosed by protein LAP and LTBP-1 in the dormant state, with one end of LTBP-1 attached to a glycoprotein fibrillin, as shown in sites (1) and (2). The glycoprotein fibronectin (purple) has affinities to the $\beta$ unit of integrin at one end and LTBP-1 at the other end (3). Stiff ECM and traction of the stellate cell both give a high chance of docking at (3). Further traction of the cell releases TGF- $\beta$ (4). The free TGF- $\beta$ is attracted by its own receptor at the site (6), as an example (Y-shape structure, green). On the other hand, traction of the cell membrane by integrins causes the ion channel Piezo 1 to open its "cover" (5). Both $\mathrm{Ca}^{2+}$ and activated TGF- $\beta$ R triggers the formation of focal adhesion (greyish-blue ellipsoid) and lining up of the actin chains (red small circles with $\alpha$-actinin). The triple-helix is a procollagen which will be secreted to the ECM, to become collagen fibril (see [2]). MMP is an enzyme to digest collagen and TIMP is a regulator of MMP; both are secreted to the ECM. Some purple triangles represent the proteoglycans and other glycoproteins in the ECM. An activated stellate cell becomes a myofibroblast; it migrates and invades with the cancer cells of the pancreas. This figure was hand-painted by author PCWF.

\subsection{Factors Triggering the Transformation from Quiescent PSCs to Myofibroblast-Like PSCs}

\section{1) Transforming growth factor}

Using rat models of fibrosis and surgical specimens from patients with chronic pancreatitis, the crucial expressions of proteins (such as $\alpha$-SMA, TGF- $\beta 1$ ) were determined in both human tissues (from necessary surgery operations) and rat specimens [176]. Pancreatic stellate cells were found to be the main source of collagen I (adjacent to the injured acinar cells) in pancreatic fibrosis in both human tissue specimens and that of experimental rat model. There was also evidence that TGF- $\beta 1$ activation of stellate cells would lead to the excessive expression of procollagen alpha $(\mathrm{I}) \mathrm{mRNA}$ [176]. More details have been included in 
the previous subsection.

\section{2) Connective tissue growth factor}

Connective tissue growth factor (CTGF, also called CCN2) is a member of the CCN family of extracellular matrix-associated heparin-binding proteins. CTGF has important roles in many biological processes, including cell adhesion, migration, proliferation, angiogenesis, skeletal development, tissue wound repair, fibrosis, and pathogenesis of several types of cancers [177]. It is known that CCN is produced at the injury site by acinar or pancreatic stellate cells, which were inferred to include a major portion of dormant PSC plus some activated PSCs also [177]. In a mouse model of alcoholic chronic pancreatitis, CTGF has been found to be secreted by activated pancreatic stellate cells [178]. There is evidence that CTGF can be activated by TGF- $\beta$. Downregulation of CTGF by the miRNA method (described in a later section) led to a decrease of activated PSC proliferation also [179].

\section{3) Platelet-derived growth factor (PDGF)}

PDGF (having 5 members in the family) is secreted by the inflammatory cells and has been shown to stimulate the activation of PSCs [180] [181]. Two PDGF receptors $\alpha$ and $\beta$ have been found in many cell types. In vitro experiments using PSCs of the rat provides evidence that the activation of PDGF receptors $\alpha$ and $\beta$ by the associated growth factors would activate the PI3K-Erk pathway (see Figure 2 and Figure 3), suggesting PDGF to be a potent mitogen for PSCs [179].

\section{4) Interleukin-10 (IL-10)}

This cytokine, which is secreted by Th2 cells, is anti-inflammatory and anti-fibrotic; it has been found that this cytokine can reach the PSCs sites. It is also found that IL-10 takes part in downregulating procollagen-1 but upregulating collagenase gene expression; the consequence is the downregulation of fibrosis [182]. IL-10 can block NF- $\mathrm{KB}$ activity [183], and is involved in the regulation of the JAK-STAT signaling pathway [184]. Over 2 decades ago, a synthetic interleukin-10 agonist was found to partly relieve acute lung injury in rabbit models with acute necrotizing pancreatitis [Osman 1998].

5) External pressure applied on PSCs induces generation of ROS, driving these cells towards the state of inducing fibrosis

The pancreas of the rat model was digested with a mixture of collagenase, and pronase which is a commercially available mixture of proteases isolated from the extracellular fluid of Streptomyces griseus [185].

The isolated PSCs were cultured and subjected to a quantifiable pressure-loading apparatus. PSCs were cultured (A) with or (B) without antioxidants (green tea polyphenol epigallocatechin gallate (EGCG) and N-acetyl cysteine) under (C) normal or (D) under high pressure of $80 \mathrm{mmHg}$. The common oxidants generated "internally" under chronic inflammation/injury are superoxide $\left(\mathrm{O}_{2}^{-}\right)$, hydrogen peroxide $\left(\mathrm{H}_{2} \mathrm{O}_{2}\right)$, oxygen molecules in the excited state; in the worst condition is a large amount of nitric oxide generated by the inducible enzyme iNOS [26] which can bind superoxide to form the cytotoxic pe- 
roxy-nitrite $(\mathrm{ONOO})^{-}$. Superoxide dismutase (SOD) and catalase are synthesized intracellularly to scavenge superoxide and $\mathrm{H}_{2} \mathrm{O}_{2}$ in the healthy state. During inflammation, endogenous antioxidants are deficient and a measure of the typical ROS level (s) is a good indication of the degree of injury roughly in general.

The main findings of [185] were: 1) Application of $80 \mathrm{mmHg}$ pressure for $1 \mathrm{hr}$ reduced SOD activity to $86.4 \%$ of control; at $2 \mathrm{hr}$ after releasing the external pressure, SOD activity was restored to $93 \%$ of control. 2) Using hydroxyphenyl fluorescein (HPF) as a detector of intracellular ROS, PSCs showed ROS even 30s after application of the stated amount of pressure. The number of HPF labeled PSCs reached a sustained maximum around $1 \mathrm{hr}$ after pressure application. 3) Application of pressure led to a significant increase in gene expressions of TGF- $\beta$, $\alpha$-SMA, and procollagen type I. 4) PSC cultured with antioxidant EGCG one hour before application of pressure practically nullified the increase in gene expressions stated in 3).

\section{Cells in the Islets Can Also Be Cancerous}

\section{The Angiogenetic Switch of the Islets}

Both cells in the acinar cells and cells in the islet can be cancerous. The following series of diagrams in Figure 15 indicates schematically the progression of the $\beta$ and the $a$ cells of islet during four stages of carcinogenesis according to the 4-stage model of [27], while the acinar/ductal cells also progress along the process of tumorigenesis. Some of the $\beta$ (bright green) and a cells (light brown) in the normal islet cells have undergone dysplasia to part (b), called hyperplastic islet, with a slight change in colors of the cells in our presentation.

Angiogenesis begins at stage (c), where cross-sections of aberrant blood vessels are grown. The transition of the stage (b) to stage (c) is called the "angiogenic switch"-this phenomenon is intuitively true because a tumor grows must be accompanied by angiogenesis. These new blood vessels supply oxygen and nutrients for further proliferation of cells in the islet, forming a large tumor with many other pathological islets in the environments (stage (d)). The a cells (secreting glucagon, some of them were painted in deeper red) were found to be colocalized with some proteoglycans, and become cancerous, a phenomenon discovered over half a century [186]. Whether the rates of cancer progression of islet cells and acinar/ductal cells are the same has not been investigated in detail, yet angiogenesis is common for all types of cancers. Since islet cells constitute only $\sim 5 \%$ of the organ mass (Section (II)), we infer that the islet cells progress with that of the acinar/ductal cells and are embedded within a lump of a tumor at the fourth stage of Figure 15, surrounded by a large stroma which contains a large amount of collagen together with their associates-proteoglycans and glycoproteins. The few $\delta$ cells (yellow), which supply somatostatin are assumed to remain normal here, as their population is only a few \% of the islet in mass and we are not aware of concrete experimental work on their way to carcinogenesis. 
The very few $\gamma$ cells, which secrete pancreatic peptide, are not shown here. Clinically, when the $\beta$ cells are progressing along a pathological path, diabetic symptoms would emerge.

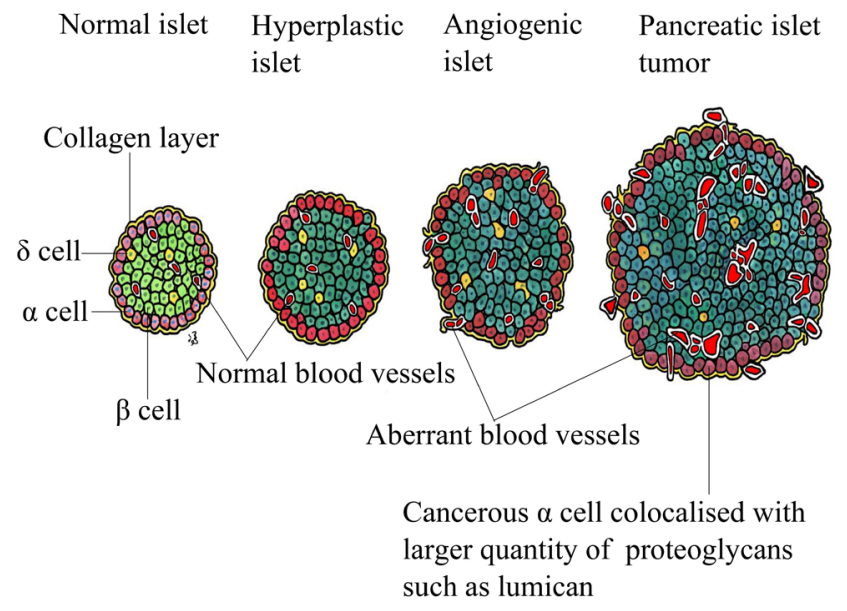

Figure 15. Progression of Angiogenic switch carcinogenesis of islet cells in the pancreas. We adapt the four-stage model of [27], with a hand-painted figure, during the progressing from a normal islet to a cancerous islet. The normal $\beta$ cells (bright green) and $\alpha$ cells (light brown) have changed to the "hyperplastic state" in (b) already, with $\beta$ cells in deeper green and a cells in bright red. The islet in stage (b) may be called or hyperplastic. Angiogenesis begins at stage (c), where cross-sections of aberrant blood vessels are grown. A large tumor is formed in stage (d), where the a cells were found to be colocalized with some proteoglycans. The a cells, which secret glucagon, were found over half a century ago capable to become cancerous also [186], and some of them were painted in deeper red at the cancerous stage. The few $\delta$ cells (yellow), which supply somatostatin are assumed to remain normal here, as their population is only a few $\%$ of the islet and we are not aware of concrete experimental work on their way to carcinogenesis. When the cancer cells begin to invade, the boundary layer of collagen (yellow) is degraded. The very few $\gamma$ cells, which secrete pancreatic peptide, are not shown here. The figure was hand-painted by author PCWF.

\section{The Small Population of Stem-Like Cells in the Pancreas Could Well Be the Earliest Metastatic Cells}

\section{Participation of the Stem-Like Cells in Pancreatic Cancer Formation and Metastasis in an Early Phase}

Note that aldehyde dehydrogenase (ALDH1) is a detoxifying enzyme responsible for the oxidation of intracellular aldehydes, has been identified as markers of stem cells of pancreatic adenocarcinomas [187]. A small population of human mammary cells was found to have a high level of ALDH1 [188]. CD24 is a mucin-like cell surface glycoprotein which may have a pivotal role in cell differentiation of different cell types [189]. CD44, a non-kinase transmembrane glycoprotein, is overexpressed in some cell types including cancer stem cells. The proteoglycan hyaluronan is the main ligand for CD44, binds to and activates CD44. In the investigation of [190], expressions of CD44, CD24 were discovered to vary with the characteristic of the local microenvironment in human pancrea- 
tic adenocarcinoma cell lines. Later, human pancreatic cancer cell lines Panc-1, BxPC3, and HPAC were used in the study of [191], and two groups of small cell populations were found to be characterized by $\mathrm{ALDH}+, \mathrm{CD} 24+$, and $\mathrm{CD} 44+$ (called group (i) cells) and ALDH-, CD24-, and CD44- (group (ii)). Group (i) cells were found to express higher levels of phosphorylated STAT3, together with translocation of STAT to the nucleus; group (i) cells also had greater tumorsphere-forming ability than group (ii) cells. This result suggests that in group (i) cells, the EGFR pathway as schematically represented by Figure 7 (EGF-Jak-STAT signaling) was active. Note that EGF can also trigger other pathways such as Akt-mTOR 1,2 signaling pathway, the LKP1-AMPK-(TSC1/ TSC2) pathway (Section (3) with Figure 2), Ras-Raf-Mek-Erk signal transduction cascade (Section (4) with Figure 3), the pathway involving translocation of EGFR to the nucleus by the dynein motor (Section (8.3) with Figure 9), plus the Hippo-YAP signaling pathway (Section (5) with Figure 5). Hence other tumorigenesis processes in addition to the EGF-Jak-STAT pathway might have been involved also. In a study using oral adenosquamous carcinoma cell line CAL 27, CD44 and EGFR were found to be colocalization by laser scanning confocal microscopy, and CD44 in fact was found to activate EGFR [192]. A few months ago, using human non-small-cell lung cancer cells, it has been found that CD44 also effectively activates EGFR in these cells [193], resulting in the activation of cell signaling pathways that induce cell proliferation, increases cell survival, modulates cytoskeletal changes, and enhances cellular motility.

In fact, more than a decade ago, three special clinical observations have been noted in the treatment of cancers of various types: 1) the appearance of metastatic lesions years after resection of small tumors with no clinically evident of metastases at diagnosis [194]; 2) detection of metastases of unknown primary tumors-such cases account for $4 \%-5 \%$ of all clinical metastases [195]; 3) it has been pointed out that only a small fraction of cells from a primary tumor enter the circulation, and less than $0.01 \%$ of these cells/cell groups develop into metastases [196]. Taken all these results together, there is ample evidence to suggest that a small population of stem-like cells play roles in pancreatic carcinogenesis. Cancer stem cells are not the only type of cells that invade. The process of invasion is of prime importance. This aspect is followed in the next several sections.

\section{Epithelial-Mesenchymal Transition and Micro-Metastasis Progression in Pancreatic Cancer}

\subsection{The Pathology of Epithelial-Mesenchymal Transition (EMT)}

Epithelial-like cells must have an organized apical-basal polarity maintained by the precise arrangement of actin filaments and adhesive molecules, such that the integrity of the organ in concern is maintained. The adhesive molecules include integrins, adherins (of which cadherin is an important example), tight junctions, other cell surface proteins, and desmosomes (structures by which two adjacent 
cells are attached, formed from protein plaques in the cell membranes which are linked by filaments) [197]. Disruption of just one or some of these surface proteins would trigger off a redistribution of molecules attached to the cell surface, including those attached to the internal surface of the membrane. There can be a disruption of the cell polarity, cytoskeletal reorganization, due to mechanotransduction [198]. The consequence is that cells are detached to become small groups or even individual cells, some of which acquire invasive power.

A similar phenomenon happens for basal cells (cells situated at the base of a multilayered tissue, as at the lowest layer of the epidermis). Thus, it is not surprising that research recognizes that, during metastasis, the cancer cells from solid tumors would disseminate into micro-metastasis entities. There is a progressive loss of epithelial cell polarity and cell-cell adhesion, and the concurrent acquisition of mesenchymal features that subsequently develop into clinically detectable metastases. This process has now coined the name "epithelial-mesenchymal transition" (EMT). Since EMT has also been found to be implicated in therapy resistance, immune escape, and maintenance of cancer stem cell properties, intermediate states might exist, as proposed in [199].

As an exocrine, the pancreatic acinar cells (PACs) produce zymogen granules which release a digestive enzyme, but the pancreatic ductal cells (PDCs) secrete water and electrolytic $\left(\mathrm{HCO}_{3}^{-}\right)$. These two types of secretions become the alkaline pancreatic juice [7]. When an injury occurs, acinar cells may transdifferentiate into PDCs, a transition called acinar-to-ductal metaphasic (ADM). The reverse transition is called DAM. If ADM is under the condition of recurrent inflammation, overexpression of KRAS or other oncogenes may bring the metaphasic state into the pancreatic intraepithelial neoplasia state (PanIN) [7]. This state is considered benign because the cells do not invade. Under a further genetic aberration such as one or more of the oncogenic pathways discussed in the previous sections, PanIN cells may develop via an EMT into pancreatic ductal adenocarcinoma PDAC [200].

\subsection{The Correlation of Solitary Cell Infiltration with EMT Using KNOWLEDGE LEARNED about EMT from Mouse Models with Mutations of the KRAS and TP53 Genes}

More recently, a special mouse model of PDAC, with pancreas-specific mutations in KRAS and TP53 genes, was used to track the fate of the pancreatic epithelial cells during various stages of pathogenesis in time [201].

One effective method to track the invasive cells was to use special lag to label an epithelial marker (such as E-cadherin, a calcium-regulated adhesion molecule that is expressed in normal epithelial tissues, including that of the pancreas; E-cadherin proteins bind cells of the same type [202]). Note that PDX1 (pancreatic and duodenal homeobox 1 ), which is a transcription factor, is necessary for pancreatic development, including $\beta$-cell maturation, and duodenal differentiation. $\mathrm{Pdx} 1^{+}$cells are almost completely apancreatic and following the track of 
Pdx1, it provides a good way to track pancreatic cancer cell invasion to other tissues [203].

Similarly, another tag was used to label a mesenchymal marker, such as ZEB1, a transcriptional repressor that has been identified as an inducer of EMT. Cells labeled with the stated two markers are "biphenotypic cells". Via the stated lineage tracing, it has been demonstrated that pancreatic cells could cross the basement membrane before invasive behavior could be detectable by standard histology, and invasive mesenchymal cells could even enter the bloodstream, as detected by flow cytometry [204]. It has been noted in another clinical study [205] that microscopic revelation shows a wide variety of intratumor glandular differentiation, with solitary infiltrating cancer cells in 114 resected pancreatic ductal adenocarcinoma specimens. A high degree of solitary cell infiltration has been correlated with reduced E-cadherin and increased vimentin expression in these specimens. Since E-cadherin is a typical adherent junction protein of epithelial cell-cell contact, and vimentin is a type III intermediate filament protein expressed in mesenchymal cells, the result highly suggests that solitary cell infiltration is correlated with EMT. The authors conclude that solitary cell infiltration would be a morphological clue to EMT and could be considered as a significant prognostic indicator of pancreatic cancer progression [205].

\subsection{Evidence of Glycoprotein Adhesion Receptor CD44 as an Inducer of Progression of EMT Based on Mouse Model Investigation}

Cells participating in EMT become mesenchymal stem cells with migratory properties; they are also considered as multipotent stromal cells that can differentiate into different phenotypes during cancer metastasis. CD44 (considered briefly in Section (14.1)), with a few isoforms, is a transmembrane glycoprotein adhesion receptor that binds hyaluronic acid in the interstitial fluid; it is involved in many cellular processes such as adhesion, growth, survival, and cell migration [206]. The primary cell lines of pancreatic tumors in iKras ${ }^{\star} \mathrm{p}^{*} 3^{\star}$ mice (a model that mimics the progression of human diseases) were employed in [207]. The findings of such study were: 1) CD44 expression in mouse pancreatic cancer correlates with EMT, with a causative link established by qRT-PCR analysis for the EMT genes (see Figure 2 of [207]). In other words, CD44s induced EMT in mice pancreatic cancer cells. 2) Short hairpin RNA (shRNA) is an artificial RNA molecule with a tight hairpin turn that can be used to silence target gene expression via RNA interference. Mouse pancreatic cancer cells iKras ${ }^{\star} \mathrm{p} 53^{\star} \# 1 \mathrm{~b}$ were infected with either (a) Control vector or (b) CD44 shRNA. Then (a) and (b) were separately injected to the pancreatic tail of NOD/SCID mice, which is a brand of immunodeficient laboratory mice, lacking mature $\mathrm{T}$ cells, $\mathrm{B}$ cells, and natural killer (NK) cells. All the animals in group (b) had large primary tumors at the site of injection. Moreover, group (a) mice showed extensive liver metastasis. 3) Expressions of CD44s were analyzed in RNA extracted from (A) re- 
sected human pancreatic cancers and (B) matched adjacent uninvolved pancreas tissues. CD44s was significantly overexpressed in the tumor specimens (see Figure 3D of [207]). 4) MT1-MMP expression was detected only in the two human cell lines that overexpressed CD44s (Figure 3E of [207]). Moreover, the application of MT1-MMP inhibition significantly decreased the invasion of the pancreatic cells on Matrigel substrate (Figure 3F of [207]). 5) Based on transcription expression analysis, the authors provided evidence that CD44s was a regulator of Snail expression. Note that Snail is a family of transcription factors that promote the inhibition of the adhesion molecule E-cadherin to regulate epithelial to mesenchymal transition (EMT) during development. The results indicate that CD44 participates in carcinogenesis at the site, most likely to be associated with the development of a favorable environment for cancer formation and metastasis. Thus, we have to be alert on the presence/activity of CD44 in cancer stem cells (Section (14)) as well as acinar cells, islet cells in the organ.

\section{The General Micro-Mechanism of Invasion of Pancreatic Cancer Cells, Stellate Cells, and Other Pancreatic Cells by the Process of Invadopodium}

\section{The Formation of Invadosomes, Secretion of} Membrane-Anchored Degrading Enzymes, Activations of Several Signaling Pathways Are Necessary for Cells to Invade through the Basal Membrane and Fascia Compartment

The basal membrane is a physical barrier between non-invasive (i.e., carcinoma in situ) and invasive types of cancer [208]. Three processes have been identified to be controlling factors as to whether cancer cells can invade through this barrier: 1) epithelial cytoskeletal contractility, 2) stromal stiffening and 3) growth factor/cytokine signaling (see Figure 1 of [208]).

The invasion of a cancer cell through the basement membrane requires the cell to acquire a motile phenotype. This cell develops actin-rich protrusive structures, called invadosomes which can degrade the first basal membrane and then the collagen fibers around the basal membrane. There are several groups of proteins/peptides involved in this complex process.

Referring to Figure 16, as an example of excitation, on the reception of epidermal growth factors (EGF, bright green hexagon) by it receptor EGFR (deep green Y-shape structure), the integrin ( $\alpha$ and $\beta$ units, brown) is activated, as indicated by the light-greyish curved arrows. Since the integrin is also attached to the basal membrane collagen by the protein fibronectin fibril (purple), mechanical stimulation can also trigger off the action of the integrin units. There are many integrins during migration/invasion in the figure. Focal adhesions are then built up around these integrin units (see Figure 3 and Figure 16).

Some key members of the focal adhesions are 1) vinculin, 2) talin, 3) paxillin, 4) Tks4/Tks5, 5) Rho A/C TPase, with 3) 4) acting as adaptor proteins. Referring to Figure 3, in brief, part of the focal adhesion organizes the polymerization of 
the actin fibers which can be attached to other parts of the cell membrane, or directly to the cell nucleus causing the synthesis of more proteins for migration. In Figure 16, we use a bluish ellipsoid to represent the focal adhesion, and a few actin chains together with the connecting proteins (i.e. the "glues") are presented simply by red tubes connecting from one focal adhesion complex to another or the cell nucleus. The calcium channel Piezo was painted in deep red. When mechanical force is applied to the integrins with focal adhesion and actin channels formed, the signal can be transmitted to the nucleus by mechanotransduction, leading to transcription of gene expressions that are associated with proliferation via the Ras/Raf/Mek/Erk and Ras/PI3K/PTEN/Akt/mTOR signaling pathways (see Sections (3) and (4)).

For cancer cells of organs (such as the pancreas) to invade, enzymes called MMPs must be in action to degrade the constituents of the ECM, leaving room for migration, in the first place. The family of metalloproteinases (MMPs) has at least 14 members. One or a small group of MMPs cooperatively degrade a variety of extracellular proteins, including the stated tightly structured collagens which are resistant to other proteases [209]. The functions of the matrix metalloproteinases (MMPs) are discussed in [2] with a summary in Table 2 of that paper.

Among these MMPs, MMP-14 is called membrane-anchored membrane-type-1 matrix metalloproteinase (MT1-MMP). The prototype protein MT1/MMP is translocated to the endoplasmic reticulum (green) after native protein transcription for "protein folding checking" with the help of chaperons (see Section (2) of [3]). It is then released from the Golgi Apparatus; an MT1-MMP complex is painted in reddish color with a fan shape structure having a zig-zag boundary.

Research in the past several years indicates that translocation of MT1/MMP is carried out by endosomes [210] [211]. Very recent evidence has suggested that the MT1-MMP complex is transported from the endoplasmic reticulum (ER) to the Golgi apparatus. There is evidence to suggest that the mode of trafficking to the cell membrane is via the anterograde transportation [212] (same as retrograde transport in Figure 9, but in the opposite direction).

Note that the protein that controls the anterograde transportation of the endosome, which carries the MT1-MMP, is found to be protrudin which is located at the ER membrane [213]. To show a simplified picture, we present a dynein motor carrying the cargo-an endosome (light yellow) containing an MT1-MMP enzyme molecule (without showing other molecules for simplicity) at site (1). The cargo is carried along one microtubule, many of those often extend from some region(s) of the cell nucleus to focal adhesions. At site (2), MT1-MMP is transported towards a focal adhesion at about the central domain of an invadosome. At site (3), a group of proteins at the focal adhesion, to be called "exocyst complex" (deep blue) that helped to expose the degrading enzyme with the active part outside the cell membrane; the MT1-MMP molecule then begins to degrade the collagen fibril (mainly collagen IV and I). Two other exposed 
MT1-MMP complexes are also shown (without painting the associated microtubules) at the tip of the invadosome, and the white zig-zag patterns represent the action of collagen degradation. Different groups of researchers found out different members of this exocyst complex; we will not review all these works, but just note that there is ample evidence that the cell membrane-anchored MT1-MMP does play a key role to degrade collagen fibrils at the basal membrane and collagen I in the ECM, leaving room for invasive cells (of which pancreatic cells are examples) to metastasize [214]. During an invasion, mechanotransduction leads to the synthesis of proteins playing roles in proliferation and invasion, as marked in the cell nucleus in the figure.

The readers are referred to different members of this complex such as N-WASH, ARF6 together with c-Jun NH2-terminal kinase-interacting protein 3 and 4 (JIP3 and JIP4) [211], GFP bound-ARF6 protein [212], TKS5, cortactin [214].

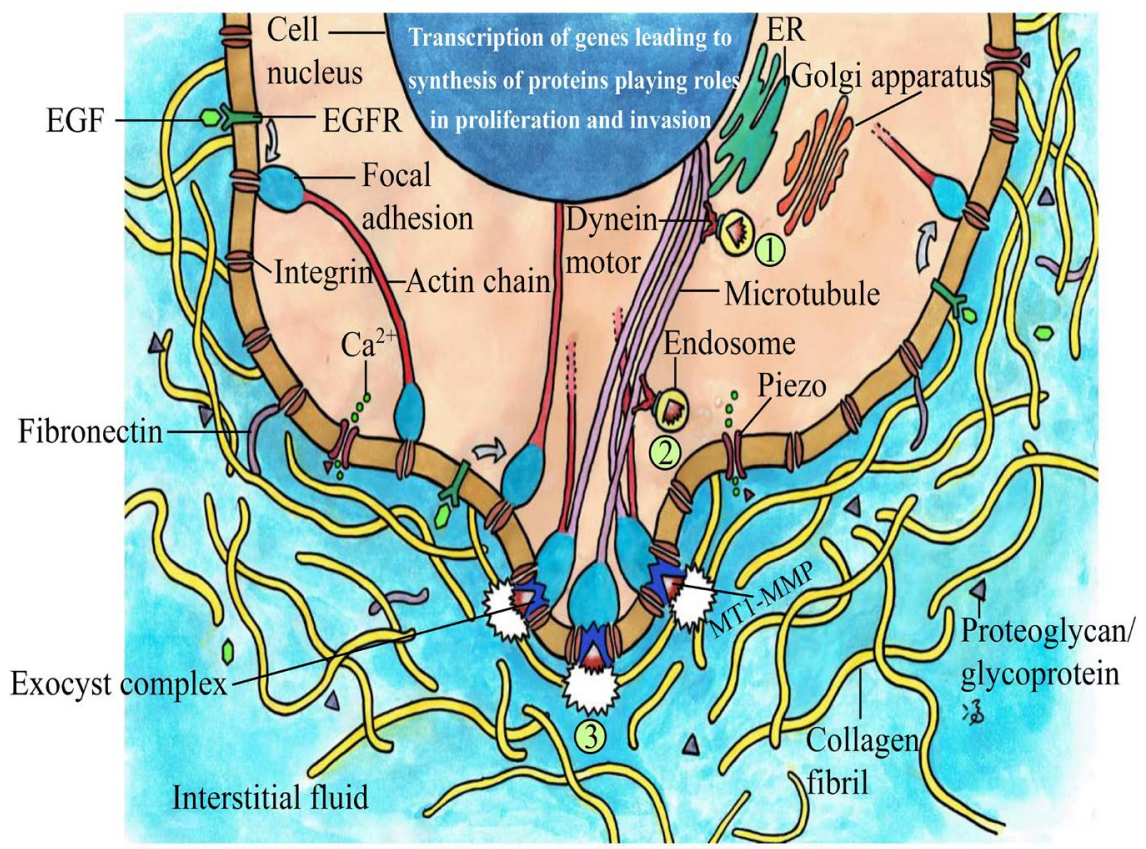

Figure 16. An invadosome is a protrusion part of a cell during an invasion. The integrins are painted in brown, the $\alpha$-SMA (F-actin) chains in red, including the proteins (such as $\alpha$-actinin) that connect the chains. The endoplasmic reticulum (green) and Golgi apparatus (orange) are labeled. The hexagon (bright green) is an epidermal growth factor (ligand) and the Y-shaped structure is its receptor. The MT1-MMP complex is enclosed by an endosome (light yellow) at site (1), after exiting from the membrane of ER. The MT1-MMP complex is carried by the molecular motor dynein along a microtubule (light purple) to site (2), towards a focal adhesion at about the central domain of an invadosome. At site (3), a group of proteins at the focal adhesion, to be called "exocyst complex" (deep blue) that helped to expose the degrading enzyme with the active part outside the cell membrane; the MT1-MMP molecule begins to degrade the collagen fibril (mainly collagen IV \& I). The degrading action is represented by white patches with the zig-zag boundary. The purple line in ECM represents a fibronectin fibril that has affinity to integrin. For other details, see text. This figure was hand-painted by author PCWF. 
In fact, the concept of invadopodium was recognized over a decade ago; see e.g. [215]. The process of invadopodium is at work when stellate cells and invasive cancer cells are in action.

\section{The Overall Picture of Progression from Normal Acinar Cells via EMT to PDAC}

\section{The Progression of a Pancreatic Ductal Tumor with a Huge Stroma with Thick Interstitial Fluid in the Facia Compartment}

We have provided the crucial updated information based on experimental evidence on the progression of normal pancreatic ductal cells via EMT to PDAC. The progression of the islets has been explained in Section (13). We summarize the result schematically in Figure 17, starting with 7 normal ductal cells on the left, without painting the islet cells. The stellate cell (orange) is in the quiescent state, and the collagen fibrils form the normal basal connective tissues with interstitial fluid (blue on the "external side" of the duct). The normal blood vessel is shown with a few red blood cells to represent schematically the normal cells found in the blood. When EMT begins to proceed, there is a change in morphology of the ductal cells, and the stellate cells start to lose the oil droplets and become activated as explained in Section (12). There are more collagen molecules synthesized and excreted to the ECM.

The pancreatic duct is under inflammation, and two macrophages are painted there to represent the presence of immunity cells in action. The development region barred by two vertical dotted lines represents the progression of the $\mathrm{Pa}$ nIN-1a, PanIN-1b, PanIN-2 to PanIN-3 states in EMT.

PanIN-3 state/stage is often called in situ carcinoma, as the cancer cells, though already formed, are not invasive. However, during this stage, some caner-stem-like cells (or simply called cancer stem cells) could have entered the upper branch of the blood vessel, as indicated by an arrow with the tip pointing to a cell (with rugged boundary). This type of metastasis is supported by the clinical finding that soon after a PDAC tumor has been resected, cancer (with evidence of pancreatic carcinoma origin) could be detected in organs at a far distance inside the body (see remarks in (Section (14.1)). We call this early metastasis, and the cancer cells are not acinar nor islet cells.

When the primary PDAC is formed, it is surrounded by abundant collagen fibrils (fibrosis), embedded in thick/viscous interstitial fluid (Section (12.2)). Antigen-presenting cells and other immunity cells are difficult to carry their duties (not painted in the figure) as they can hardly reach the core of the cancer-stroma domain. Ductal cancer cells are detached from the tumor and migrate to the fascia compart of that region, with some enter the blood vessel, as indicated by the arrow on the right-lower part of the figure.

There are proteoglycans and glycoproteins in the fascia compart, and they are represented by greyish-purple triangles.

The bright green circles represent the drug molecules (e.g. chemotherapeutic) 
which can only physically reach the outside boundary of the stroma, as the interstitial fluid pressure inside the stroma is very high - this is a well-known fact expressed as drug resistance in clinical treatment. Radiotherapy sends electromagnetic energy which is absorbed by the stroma and healthy cells on route to the supposed target. In Figure 17, the white small circles in the interstitial fluid, blood are vesicles containing important micro-RNA information of the sending cells; this issue will be discussed in later Sections.

By the way, the purplish-blue domain above the ductal cells represent the digestive enzymes passing towards the duodenum, and the greenish patch at the upper right-hand corner represents some bile, as both the pancreatic duct and bile duct enters the duodenum in the close neighborhood.

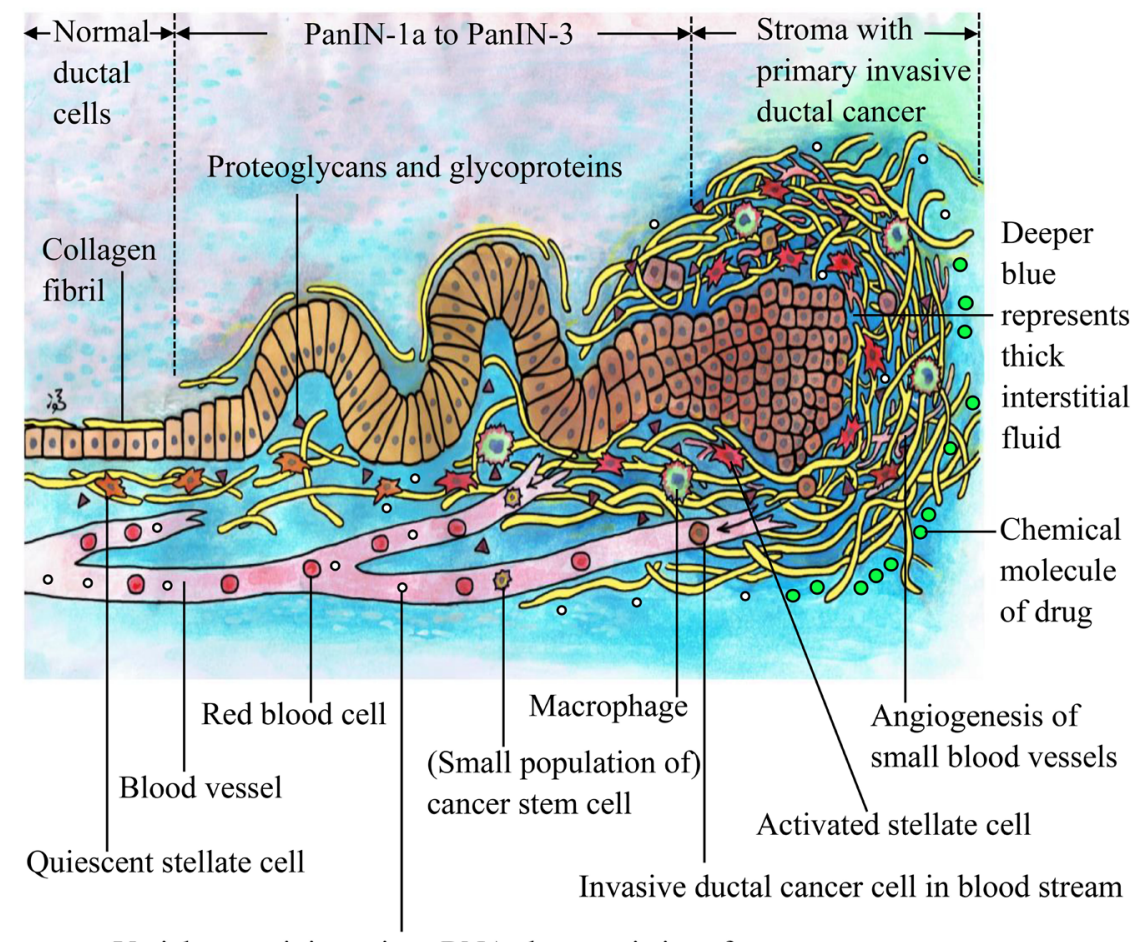

Vesicle containing micro-RNA characteristics of the status of the sending-cell

Figure 17. The overall picture of carcinogenesis of PDAC. The brownish cells are ductal (acinar) cells that progress from normal structure to a lump of tumor surrounded by dense collagen fibrils (fibrosis) and viscous interstitial fluid (deep blue). Several macrophages represent the presence of immunity cells. The quiescent stellate cells (orange) change to myofibroblast-type of activated stellate cells (red). There is a small population $(<5 \%$ in body mass of the organ) of cancer-stem-like cells that could migrate to the bloodstream during the PanIN-3 stage. Losing physical connection with the ductal cells, individual ductal cancer cells can migrate via the blood, interstitial fluid to other internal organs. Drug molecules (green circles) and radiotherapeutic energy cannot reach the primary tumor because of the high interstitial fluid pressure and dense collagen fibrils around the stoma. The white circles are vesicles containing information of non-coding micro-RNAs sent by all cells for cell-cell communication via the fluid system of the body-this important aspect will be followed up later. This figure was hand-painted by author PCWF. 
18. Having Analyzed the Pathogenesis of Pancreatic Cancers, in Addition to Those Considered at the Ends of Previous Sections Related to Signaling Pathways, the Following Are Examples of Therapeutic Measures, Including Examples of Non-Coding RNAs, as Potent Treatments

\subsection{Prevention of Stellate Cell Activation and Efficacy of Treating Pancreatic and Gastrointestinal Xenografts with ATRA, the Main Constituent of Vitamin A}

Retinoids are a class of biomolecules having similar structural with respect to chemical activity of vitamin A. All-trans-retinoic acid (ATRA) is an oxidation product of retinol which exists only in small amounts in food. Taken orally, ATRA is rapidly absorbed in the digestive system, bound to serum albumin in the blood. Whereas vitamin A from food is first stored in the liver, ATRA is rapidly metabolized and excreted in the bile. This type of metabolism avoids the side effects of taking too much Vitamin A (hypervitaminosis A), leading to the symptoms of skin desquamation, vomiting, and diarrhea [216].

To study the effect of ATRA on human pancreatic cancer as the main goal, two human pancreatic cancer cell lines were cultured: 1) Panc-1,2) MIA-PaCa-2, as well as 3) carcinoid cell line (BON) - derived from the gastrointestinal tract, plus 4) the rat insulinoma cell line RIN- $5 \mathrm{mF}$ (a clone derived from the RIN-m rat islet line (ATCC CRL-2057)). Different amounts of ATRA were cultured with these cell-lines [217].

BON cells were injected into the healthy mice model to introduce carcinogenesis as in vivo study. The resulting tumor xenografts were then harvested and the number of viable tumor cells was counted. In the in vitro investigation, a large amount of ATRA (100 micro-M) had to be used to reduce the viable cancer number to that of the control, whereas an amount of 0.5 to 1.0 micro-M of ATRA was needed to reduce the viable cancer cells to the control for cell lines 1), 2) and 4) (see Figure 1 of [217]). The size of the BON-induced tumor of the in vivo model was also found to decrease in size (from $175 \mathrm{~mm}^{2}$ to $70 \mathrm{~mm}^{2}$ ) after 37 days of treatment (see Figure 4 of [217]). This section deals with the beneficial result of treatment of cancer cells with ATRA.

In another study, human pancreatic stellate cells (PSCs) were cultured on 3D Matrigel and the magnetic tweezer micro-rheology technique (with fibronectin-coated beads) was employed to analyze the contractility ability of PSCs by measuring the displacements of the magnetic beads [218]. Through the detection of the expressions of alpha-smooth muscle and vimentin, which are two basic markers of myofibroblasts activation, it has been reported that 1) these two expressions were decreased when PSCs were treated with ATRA (see Supplementary Figure 4 of [218]. 2) The expression of another protein desmin, which is an established marker of PSC quiescence, was found to decrease. These two results suggested that ATRA would suppress the ability of the PSCs to sense the mechanical cues of the ECM, so that the PSCs would remain in the quiescent state, 
avoiding the mechanical vicious cycle mentioned in Section (12.2).

Note that myosin light chain-II (MLC-2) is part of the functional motor protein myosin. Activation of MLC-2 is necessary to produce traction force. The mechanism of ATRA action in keeping PSCs in the quiescent state was inferred to be due to transcriptional suppression of MLC-2 [218].

Human tumor tissues were obtained from PDAC patients and mouse xenograft models with the injection of human cells Panc02 were established in [Bleul 2015]. The concentrations of ATRA and levels of ATRA receptors (RAR $a$ and $\operatorname{RAR} \beta$ ) were reduced in tumor tissues as compared to normal.

In a recent investigation, using nano-gold particles to deliver ATRA to PSCs, it has been reported that activated PSCs could be brought back to the quiescent state, suggesting that such a method might be used as a therapeutic treatment in the future [174].

\subsection{Three Members of Tocotrienols (a Subfamily of Vitamin E) Were Found to Suppress the Activity of NF-kB in Pancreatic Cancer Cells}

Tocopherols, found commonly in food, function as antioxidants by donating $\mathrm{H}$ atoms to radicals. The four members of the tocotrienols, a subclass of vitamin $\mathrm{E}$, are also antioxidants and found to be derived mainly from palm oil. Tocotrienol rich fraction (TRF) is an extract of palm oil, which consists of $25 \%$ alpha tocopherol ( $\alpha$-TCP) and 75\% tocotrienols (with sub-types $\alpha-, \beta-, \delta-, \gamma$ ) [219] [220].

The tocotrienols are similar to the tocopherols in chemical structure but with carbon bonding shifts in three sites; these two sub-classes of Vitamin $\mathrm{E}$ have homologs named according to the degree of methylation at R1 and R2.

Tocotrienols of types $\alpha-, \gamma$ - and $\delta$ were detected in both plasma and lipoproteins with $\alpha$-tocotrienol as the major homolog after administration of TRF. It was remarked particularly that tocotrienols were mainly detected in the HDL cholesterol at 4 to 8 hours before clearance from the body [220]. The small HDL particles selectively distributed a-tocotrienol to organs and tissues with high adipose content. On the other hand, tocopherols were distributed equally in all the lipoprotein fractions; since LDL receptors are present in all tissues, tocopherols are found in all tissues and for a time longer than 8 hours. It is thus not surprising that a special delivery system is needed to bring tocotrienols to cancer sites.

When administered orally, the amount of absorption of vitamin E depends on the degree of lipolysis and food intake, as it is water insoluble. With tocotrienols embedded in nano-carriers aimed at targeting tumor, intravenous administration via tail vein injections confirmed that the modality of administration did affect significantly the degree of absorption [221]. Using hydrophilic polymers (such as cyclodextrin) and emulsifiers (such as polysorbate 80, polyoxyethylene sorbitan monooleate (Tween 80 )) as "drug delivery systems", it has been shown that the concentration of $\gamma$-tocotrienols in plasma would be increased in rat 
models (see e.g. [222]). Employing murine xenografts created by injection of human cancer cells, there is evidence that tocotrienols therapy can be a very promising one to eradicate breast, pancreatic, and prostate cancers when encapsulated in a tumor-targeted vesicle system [223].

In another study, human pancreatic ductal epithelial cells (HPDE6 C7) and HPDE6C7-KRas cells were used in [224]. These two cell lines were grown in keratinocyte growth media (Invitrogen) supplemented with human epidermal growth factor and bovine pituitary extract. Using the Cell proliferation MTT assay on human pancreatic cancer MiaPaCa2 and AsPc-1 cell line in vitro, it has been demonstrated that natural tocotrienols with d-tocotrienol and $\gamma$-tocotrienol caused cancer cell viability reduction up to $60 \%$ with $50 \mathrm{mmol} / \mathrm{L} ; \beta$-tocotrienol has a moderate anti-cancer effect with cancer cell viability reduction up to $40 \%$ with $50 \mathrm{mmol} / \mathrm{L}$ (Figure 1B of [224]). With human pancreatic cells AsPc-1 injected into the mice in vivo models, it was found that d-tocotrienol significantly reduced tumor volume by $50 \%(\mathrm{p}<0.001), \gamma$-tocotrienol reduced tumor volume by $42 \%(\mathrm{P}<0.01)$, and $\beta$-tocotrienol reduced tumor volume by $32 \%(\mathrm{p}<0.01)$, whereas $\alpha$-tocotrienol did not decrease tumor size growth significantly. The in vitro (human cells) and in vivo (mice) studies substantiated each other in general.

The authors particularly pointed out that in the in vitro investigation, 1) $\gamma$-and d-tocotrienols inhibited the NF-kB activity in the nuclear extract, 2) whereas $\gamma^{-}, \delta$ - and $\beta$ - tocotrienols inhibited the NF-kB activity in the extract obtained from the cytosol (see Figure 3A of [224]), but 3) $\alpha$-tocotrienol and $\alpha$-tocopherol were found to be ineffective in reducing NF-kB activity. More recently, using human pancreatic cell lines $\mathrm{L} 3.6 \mathrm{pl}$ and MiaPaCa-2, plus orthotopic xenograft mouse models, both cancer volume growth and cancer stem cells (with $\mathrm{CD} 44$ as a marker) activity have been suppressed by the natural form of tocotrienols, preventing metastasis [225].

\subsection{Agonists of Vitamin D Receptor Reduce Crosstalk between Pancreatic Stellate and Pancreatic Cells-VDR Ligand Calcipotriol (Cal) Treat Pancreatitis and Pancreatic Cancer}

The pancreatic stellate cell was discovered as peri-acinar cells in the rat with a stellate shape when activated [168]. Based on the heatmaps representing selected genes from RNA-sequence analysis of 1) quiescent mouse PSCs and 2) activated mouse PSCs (isolated from the animals), the authors have discovered that certain genes are overexpressed in 2). These genes correspond to the proteins participating in forming focal adhesion, gap junction formation, matrix remodelling, inflammation [226]. Specimens of PSCs were obtained during resection surgery of non-cancer associated and cancer-associated patients in the same study. A comparison of the transcriptomes of non-cancerous human specimens and cancerous human specimens gave similar results, pointing to the fact that PSCs actively participate in matrix remodelling during carcinogenesis and me- 
tastasis of pancreatic cancer.

These findings substantiate the suggestions/conclusions of other studies, pointing to the fact that PSCs actively participate in matrix remodelling during carcinogenesis and metastasis of pancreatic cancer.

The more important discovery in [226] is the revelation of vitamin receptor (VDR) in the stroma of pancreatic cancer, rather than in acinar cells, with a minimal amount in the islet, based on quantitative RT-PCR investigation. Human pancreatic cell lines (MiaPaCa-2, BxPC-3, HPAC, Panc1, APC1) study demonstrated that VDR activation drives the reversion of PSCs to a more quiescent state. More precisely, the application of VDR ligand calcipotriol (Cal, as a non-hyper calcemic vitamin $\mathrm{D}$ analog) caused a decrease in markers of inflammation, markers of fibrosis in pancreatitis, and human pancreatic tumor stroma samples. Cal treatment was found to induce liquid droplet formation in 19/27 primary cancer samples from patients. The authors consider that as VDR behaved like a master transcriptional regulator of PSC activation network; targeting the receptor with vitamin $\mathrm{D}$ as a ligand would drive the activated PSCs to their rest state [226]. The authors remarked also that the addition of Calcipotriol to gemcitabine treatment enhanced survival of the "KPC mice" model by $58 \%$. The KPC mice model has KRAS, TP53 mutations to promote chromosome instability with widely metastatic ability [227].

In recent work, 3 immortalized stellate cell lines were generated from pancreatic patients [228]. Exosomes released by stellate cells were labeled with a fluorescent dye and incubated with human pancreatic cell lines PANC-1 and SW1990. The exosomes received by the cancer cells with efficiency $>90 \%$. Exosomes from different stellate cells showed similar miRNA expression profile with each other. Five most abundant miRNA in exosomes were miR-10a-5p, 92a-3p, 181a-5p, 191-5p, 92b-3p. Vitamin D receptor signaling (supply of Vitamin D to the stellate cells) could suppress secretion such as miR-10a-5p, implying that messages from the stellate cells to the cancer cells were influenced.

\subsection{Drugs to Decrease the Concentration of the Interstitial Fluid in the Stroma-Pegvorhyaluronidase Alfa (PEGPH20), together with Other Anti-Cancer Drugs Aiming to Treat PDAC}

Note that hyaluronan (hyaluronic acid, HA), which is the most abundant and ubiquitous member of the glycosaminoglycans (GAGs, a member of proteoglycan), forms negatively charged long chains in the fascia. The hydronium ions together with layers of water (originated from the bloodstream) are then bound to the hyaluronan chains, forming a thick interstitial fluid (IF) [2]. HA, secreted by cancer and stromal cells, binds to and interacts with specific cell surface receptors including CD44 (a surface marker for cancer stem cell), and receptor forming HA-mediated motility (receptor) (abbreviated RHAMM). It is not surprising that HA, which is also secreted by cancer cells, has been shown to be abundantly accumulated in the surrounding stroma of tumor. The concentration 
of HA is about 12 -fold in PDAC as compared with that of normal pancreas. HA takes part in strong tumor cell-stroma interaction. Thus we can view the concentration of HA in the ECM of the pancreas as a sign of cancer initiation and progression [229]. Stromal modifying agents, such as Pegvorhyaluronidase alfa (PEGPH20), which can (temporarily) degrade HA, in combination with nab-paclitaxel plus gemcitabine (NCT01839487) together with modified FOLFIRINOX, is in clinical trials for patients with metastatic PDAC [230] (see also the review of [229]). Though we have not heard about positive results by 2019, the general idea is scientifically sound. The reader is also referred to the work on enzymatic targeting HA-it was in preclinical animal trials by 2010 [231].

\section{MicroRNA Therapy to Treat Pancreatic Cancers in General up to 2015}

\subsection{How Does the microRNA Therapy Work?}

A microRNA (abbreviated miRNA) is a small non-coding RNA molecule (containing about 20 nucleotides) which functions in RNA silencing and post-transcriptional regulation of gene expression via DNA interference.

The interaction of miRNAs with their target genes is dynamic and dependent on many factors, such as the subcellular location of miRNAs, the amounts of miRNAs and target mRNAs present, plus the affinity of miRNA-mRNA interactions. The miRNAs can be secreted into interstitial fluids and transported to target cells via vesicles, such as exosomes. Thus cell-cell communication can be mediated by miRNAs that function as chemical messengers through the ECM [232].

The role played by miRNA has drawn much attention during carcinogenesis. For example, miR-320 has been reported to have an inhibitory effect on gastric cancer progression and being taken clinically as a novel biomarker for its diagnosis and prognosis [233]. As another example, transcriptome profiling of the epithelial components of micro-dissected ovarian tumor (as an example of epithelial cancer) samples isolated from long- and short-term survivors has been carried out. Then it was found, via a miRNA transfection process, that there was a direct binding of miR-320 to the 3 ' untranslated region of mRNA of the transcription factor called "E74-like factor 3 (ELF3)", arriving at the "silencing effect". The authors concluded that the protein ELF3 would be a negative regulator of epithelial-mesenchymal-transition (EMT) of the ovarian tumorigenesis [234]. Furthermore, using specimens from 52 breast cancer (BC) patients, it has just been demonstrated [235] that miR-320 targeted ELF3 would suppress BC cell progression via regulation of PI3K/Akt signaling, the details of which have been reviewed in Section (3). Since all of the above cancers are epithelial in nature, it would be fruitful to look for the same/similar miRNA silencing mechanism in pancreatic cancers.

The detailed mechanisms of the interactions between miRNAs and the identi- 
fication of the targeted transcriptors/proteins which are involved in tumorigenesis are certainly useful in developing therapeutic measures. In this new process, first, using the transcriptome profiling technique, scientists look for one or more transcription factor-encoding genes whose expressions are high in certain cancer samples. With the relevant cancer cell lines, the miRNAs are applied to silence such transcription factors and analyze the progress of carcinogenesis in these cell lines; after that, it is fruitful to establish xenograft in vivo models to analyze the anti-cancer effects.

\subsection{MiRNA96 and the Ras-Mek-Erk Pathway}

The three human RAS genes have members KRAS, NRAS, and HRAS. KRAS is the most frequently mutated oncogene, appearing in $90 \%$ of pancreatic cancers [236] and is one of the earliest genetic alterations detected in pancreatic cancer development. KRAS encodes small GTPases that are involved in cellular signal transduction. Oncogenic KRAS has been established to promote pancreatic tumorigenesis through activation of (multiple) downstream pathways, including phosphatidylinositol 3-kinase (PI3K)/Akt [237], extracellular signal-regulated kinase (Erk, see Figure 3), and NF-кB [238]. The protein Ras has been observed in rat's pancreatic ductal cell line to elicit mitogenic and morphogenic responses, with the result of increasing S-phase cell population [239].

In the study of [240], specimens of tissues were obtained from patients receiving surgical operation for pancreatic cancer. Three pancreatic cancer cell lines 1) MIA PaCa-2, 2) PANC-1, 3) BxPC-3 and 4) the well-known cervical adenocarcinoma cell line Hela were used in the in vitro investigation. Total RNA was extracted from the cells and tissues. Real-time reverse transcription PCR assay was conducted to detect the mRNA levels of KRAS and glyceraldehyde-3-phosphate dehydrogenase (GAPDH). Human KRAS mRNA was cloned using a PCR-generated fragment.

The findings of this investigation [240] using the above preparations were; a) Northern blotting analysis was conducted in 10 pairs of pancreatic cancer tissues and matched adjacent normal tissue samples indicated that the expression of miR-96 was lower in all the pancreatic cancer tissues than in normal tissues. b) Expression miR-96 was downregulated in tumor cell lines 1) to 3).

c) adopting three bioinformatic algorithms, a large number of potential target genes of miR-96 was identified. Among these candidates, a binding site of miR-96 was observed in the sequence 3'-UTR of KRAS mRNA, with perfect base pairing. d) Using "CCK-8 proliferation essay" (for details see [240]), overexpression of miRNA in cell lines 1) and 2) inhibited cell proliferation compared to control (untreated cells transfected with "miRNA scramble" sample). e) Pre-miR-96 treatment induced cell cycle arrest in G1 phase, with detected decrease in population of the $S$ phase. $f$ ) Using artificial wound model in vitro, the introduction of miR-96 into cell lines 1) and 2) resulted in a reduction of cell migration inclosing the artificial wound cells. We note that the Ras-Mek-Erk 
pathway in Figure 3 is related to the Akt/mTOR pathway (Figure 2) via cross-talks.

\subsection{Promise of micro-RNA34 Mimics to Treat Pancreatic Cancers with TP53 Mutation by Targeting the Notch Pathway and Anti-Apoptotic Bcl-2 Protein}

In a normal pancreas, the Notch signaling pathway has been shown, using the mice model, to be an important regulator of the balance between self-renewal and differentiation, involving both the endocrine (islet) and exocrine (acinar) cells [12]. Activation of the Notch pathway prevents the differentiation of pancreatic acinar cells and attenuates endocrine development [241]. The result of suggested the Notch Pathway is necessary to maintain the cancer stem cell population in pancreatic cancer cells as explained in [162].

On the other hand, the tumor suppressor gene p53 was found to regulate directly each of the three family members of the micro-RNA miR-34a,b,c (see e.g. [242]), which targets Notch pathway proteins. Moreover, there is crosstalk between the Notch pathway the Bcl-2 proteins which are the regulators (anti-apoptotic) of (intrinsic) apoptosis [243].

Given the above logical connection, the effects of this mi-RNA-34 on cancer cells became the focus of study in [244]. Five pancreatic cancer cell lines with p53 mutations were used in [244]: 1) Mia PaCa2; 2) BxPC3; 3) Capan1; 4) Capan2; 5) Panc-1. A normal human lung fibroblast cell line WI-38 was also employed.

Further, mice models transfected with a) cells of 1), and b) cells of 1) plus miR-34(s) were established. Their findings were i) The levels of miR-34 in cancer cells were in general lower compared to lung fibroblast cells. ii) Tumorsphere-forming and tumor-initiating cells (with surface markers CD44 and CD133), called cancer stem cells, were identified in 1). These stem cells showed high levels of Notch/Bcl-2, but a loss of miR-34s. iii) Transfection of miR-34 mimics to 1) and 2) inhibited expression levels of Bcl-2, Notch1, Notch2. iv) Restoration of miR-34 to cells 1) and 2) showed significant inhibition of clonogenic cell growth (forming colony of cells) and invasion, accompanied by more apoptosis and cell cycle arrest. v) Restoration of miR-34 to cells 1) led to an $87 \%$ reduction of cancer stem cells. vi) miR-34 mimics would partly restore the tumor-suppressing function of $\mathrm{p} 53$ in cells 1)-4) with deficient $\mathrm{p} 53$. vii) In the in vivo mice model b) stated above, restoration of miR-34 inhibited tumor formation.

\subsection{Using Inhibitors of microRNA21 and microRNA 221 to Treat Highly Metastatic Pancreatic Human Cancer Cell Lines, Resistant to Gemcitabine Using in Vitro and in Vivo Mouse Models}

By the early $21^{\text {st }}$ century, it was confirmed that a small population (SP) of cancer stem cells existed in cancers [245]. In [246], a highly metastatic human pancrea- 
tic cancer cell line 1) L13.6pl (which contained the cancer stem cells); 2) a small population of cancer cells were isolated following Hoechst 33342 staining of 1); 3) L13.6pl cells with cancer stem cells removed-called non-SP cells; and 4) L3.6pl cells $s_{\text {Gres }}$ (gemcitabine resistant cells cultured with increasing concentrations of the drug gemcitabine). Note that an earlier study already indicated that anti-sense oligonucleotides (ASOs) of microRNA-221 and microRNA-21 would produce antiproliferative effects on human pancreatic carcinoma cell lines [247]. Other cell lines were constructed readily: 5) L13.6pl cells transfected with anti-sense miR-21; 6) L13.6pl cells transfected with anti-sense miR-221; 7) L13.6pl cells transfected with anti-sense miR-221 plus anti-sense miR-21. For in vivo study, cells of specimens 1), 4), 5), 6), 7) were injected into mouse models to investigate the development of cancer. The following results were highlighted: a) Expressions of miR-21and miR-221 were high in sample 1) compared with that of the sample 3). b) Expressions of miR-21and miR-221 were significantly upregulated in the sample 2) as compared to 3). c) The content of SP in 1) was 5\%; that of the sample 5) was reduced to $4.7 \%$; that of the specimen 6) was reduced to about $3 \%$; that of the specimen 7) was further reduced to about $2 \%$. d) There was no change in viability cell number in the specimen 5) as compared to 1). e) The viability number of 6 ) was reduced to about $85 \%$ as compared to 1 ). The viability number of 7) was reduced further to about $50 \%$ as compared to 1 ). The in vivo study indicated that the combined anti-sense therapy of (miR-21 plus miR-221) gave the best result in tumor size reduction. The above experimental result indicates that inhibitors of miRNA21 and miRNA221 together might be an effective therapy to treat pancreatic cancers which are resistant to gemcitabine.

\subsection{Evidence of Suppressing Cancer Progression Employing miRNA 146a, 3,3'-Diinolyl-Methane, Natural Products Isoflavone G-2523 Based on Human Cell Lines Study-Leading to Downregulation of EGFR, Downregulation of NF-kB, Upregulation of PTEN}

Two human pancreatic cancer cell lines 1) Colo357, 2) Panc-1, 3) human pancreatic ductal epithelial cells (HPDE) were used in [248]. Two types of treatment were tried: a) 3,3'-diinolyl-methane (DIM), a natural product derived from vegetables like broccoli, Brussels sprouts, cabbage; b) isoflavone G-2535 (Genistein), a soy phytoestrogen from soy, green peas, alfalfa, peanut. The findings of [248] are: i) Both a) and b) activate miR-146a. ii) Levels of miR-146a in HPDE are higher than that in each of the cancer cell lines. iii) Treatment of 1) and 2) cells with a) and b) increases expressions of miR-146a. iv) When cells 1) and 2) were transfected with miR-146a pre-micro-RNA, the expression of epidermal growth factor receptor (EGFR) was downregulated at mRNA and protein levels. v) Anti-miR-146a was transfected into 1) and 2); the EGFR level was found to increase after such inhibition of miR-146a. vi) When cells in 1) and 2) were transfected with miR-146a, there was a decrease of the expression level of NF-kB. vii) 
Moreover, the expression of metastasis-associated protein MTA-1 was downregulated after the stated transfection in $\mathrm{v}$ ).

Note that there is recent research reporting that overexpression of MTA 2 was detected in 64 PDAC tissues [249]. Through an in vitro study using human pancreatic cell lines MiaPaCa-2, PANC-1, and mouse xenograft in vivo investigation, MTA2 was negatively correlated with the anticancer protein PTEN (see Figure 2). Benzyl isothiocyanate (BITC) which is found in cruciferous vegetables; BITC has been used as a chemoprotective agent against carcinogenesis. There was evidence showing MTA2 and PTEN played roles in the regulation of the PI3K-Akt pathway in BTIC-mediated cancer suppression [249]. Collecting the above information, we can forth the notion as written in the title of this section.

\section{Early Non-Invasive Detection of Pancreatic Cancer in Body Fluids-From Micro Non-Coding RNAs, Circular Non-Coding RNAs to Long Non-Coding RNAs}

\subsection{Introduction to Three Types of Non-Coding RNAs-They Are Enclosed by Vesicles, Carrying Physiological/Pathological Signals, and Should Be Found in Body Fluids}

Almost all cell types known (including cancer cells) abundantly release small "particles" known as extracellular vesicles (EVs or called exosomes) into the extracellular milieu for cell-cell communication, at near and far distances through the largest organ of the body-the fascia. The "messages" in these EVs contain nucleic acids (including microRNAs), proteins, lipids, and carbohydrates, characteristics of the cell type, and status. The term exosomes are now defined as extracellular vesicles that are released from cells upon fusion of an intermediate endocytic compartment. In fact, transport of exosomes is like the artificial nano-particle drug delivery system that has recently been developed.

Several years ago, using high-throughput RNA sequencing (RNA-Seq), an in-depth bioinformatic analysis of non-coding RNAs (ncRNAs) in human cell-free saliva (CFS) from healthy individuals was conducted [250]. A "common ncRNA" (i.e. miRNA) has only up to about 20 nucleic acids, having their free 3' and 5' ends exposed and can be degraded by nucleases. Note that circular RNA (circRNA) is a segment of (single-stranded) RNA that forms a covalently closed continuous loop. Through binding with miRNAs, and other gene expression regulators, together with circRNA regulate various biological processes in the human tissues (see e.g. [251]). Note that a circRNA cannot be digested readily by nucleases due to its ring structure. Since a circRNA is stable in the body for a longer time, it is of interest to detect such units for diagnostic purposes, specifically for various diseases. The circRNAs are also called "sponge" RNAs which interact with the common microRNAs due to their loop structures [252]. The piwi-interacting RNAs (piRNAs) which are available in embryonic stem and skin cells were also detected. These RNAs are also of interest clinically in the 
early stages of an epithelial type of cancers, of which pancreatic and breast cancers are typical ones. A non-coding RNA composed of more than 20 nucleic acids is called lncRNA, the research on which has also become a hot topic in the recent few years.

Note that the non-coding RNAs have been detected in the cerebrospinal fluid also [253]. Most studies so far have been focussed on their detection in the bloodstream.

Four years ago, we have provided evidence that the interstitial fluids, cerebrospinal fluid, glymphatic fluid, lymphatic fluid, blood, and other fluids form a circulating fluid system of the body [166]. Saliva and sweat are in fact parts of the interstitial fluid. We predict that various types of non-coding RNAs will be detected in the interstitial fluids in different compartments of the body, because the fluids mentioned form a circulation system, with input as drinks through the mouth, and discharged as urine. Indeed, several months ago, for renal transplant patients with acute $\mathrm{T}$ cell-mediated allograft rejection, it has been reported that the circular RNA "has_circ_0001334" is enhanced in the urine of these patients [254]. Non-invasive detection of such a circular RNA becomes a biomarker of acute kidney rejection; the technicality of the method of measurement is described in that paper.

In an "earlier" study, the expression profile of circRNAs in six PDAC cancer specimens and associated adjacent normal tissues using microarray has been analyzed [255]. A high-throughout circRNA microarray was used to identify dysregulated circular RNAs in specimens of these six PDAC patients and the authors have concluded that clusters of circRNAs are aberrantly expressed in PDAC compared with the normal specimens [255].

\subsection{The Non-Coding Circular RNA IARS Enclosed within Exosomes Regulates Endothelial Monolayer Permeability to Promote Pancreatic Cancer Metastasis Based on Human Pancreatic Cell Line and Xenograft Investigation-Looking for Antisense circRNA IARS}

In the study of [256], RNA was isolated from 85 fresh frozen tissues and 16 peritumoral normal tissues of 92 patients underwent pancreaticoduodenectomy surgery. The human pancreatic cancer cell lines AsPC-1, Hs 766 T, plus the human microvascular vein endothelial cells (HUVEC) were also used. qRT-PCR was employed to measure the "circulating" Circular RNA (circRNA) IARS (enclosed by vesicles) secreted by the pancreatic cancer cells. Expressions of proteins related to focal adhesion and F-actin were also determined in the relevant specimens. The endothelial monolayer permeability was estimated. Human pancreatic cells were injected into the mouse model for in vivo study of cancer development. They found that [256]: 1) The circulating vesicles enclosing microRNA increased endothelial monolayer permeability (by enhancing expressions of the F-actin and focal adhesion proteins) and the vesicles entered the venous blood. 2) Based on the in vivo investigation, the circRNA IARS was 
found to be correlated to tumor invasion and metastasis. 3) Overexpression of circRNA IARS significantly down-regulated miR-122 and ZO-1 levels. We would remark that ZO-1 is a tight-junction protein acting for cell-cell adhesion [257]. For a group of cancer cells to invade, the migrating cell must be detached, by breaking cell-cell adhesion.

There is evidence that the expression of miR-122 mediated by adenoviral vector induces apoptosis and cell cycle arrest of (liver) cancer cells [252]. Intuitively the "absorption" of miR-122 and ZO-1 by the circRNA IARS would promote cancer micro-metastasis, as observed in [256]. Given the above analysis, the application of anti-sense non-coding circular RNA IARS would reduce endothelial permeability to decrease the ability of invasion of cancer cells.

\subsection{The Expressions of Non-Coding Circular RNA LDLRAD3 Are Upregulated in Human Tissues, Plasma Samples, and Human Pancreatic Cell Lines}

Expression levels of circ-LDLRAD3 were determined in normal pancreatic cell lines (HPC-Y5 and HPDE6-C7) and pancreatic cancer cell lines (Capan-2, Panc-1, SW1990, and AsPC-1) [258]. Cancerous tissues and nearby non-cancerous pancreas tissues were obtained from 30 patients. Thirty-one sets of blood samples were obtained from these patients and a group of healthy subjects. Analysis of these specimens indicated that the expression levels of a specific circular RNA (circ-LDLRAD3) were higher in pancreatic cancer cell lines, pancreatic cancer specimens, plasma samples from pancreatic patients than in normal pancreatic cell lines, non-cancerous specimens, plasma samples from healthy normal respectively $(\mathrm{p}<0.01)$ [258]. Anti-sense non-coding circular RNA LDLRAD3 might be a potent treatment of PDAC.

\section{4. circRNA_100782 Regulates PDAC Cell Growth by Affecting IL6-Jak-STAT3 Signaling Pathway}

In the study of [259], the authors have used the PDAC cell lines BxPC3 and HPDE and HEK293T cells, plus pancreatic cancer mice models to study tumor status in vivo. Based on xenografts analysis, they have found that circRNA_100782 regulates PDAC cell growth by affecting IL6-Jak-STAT3 signaling pathway and this pathway promotes tumor growth as briefly explained in Section (8) with Figure 7. circRNA_100782 knocked down models repressed tumor growth. Therefore, inhibitor of circRNA_100782 is a potential treatment for PDAC.

\subsection{Non-Coding Circular RNA circ_0007534 Plays an Oncogenic Role}

In another recent study [260], real-time (RT-qPCR) was used to detect the expression of circ_0007534 in 60-paired PDAC tissue samples of subjects. 5 human PDAC cell lines (BxPC3, Capan-2, AsPC-1, PANC1, and SW1990) were used to detect cell proliferation, apoptosis, and metastatic properties affected by circ_0007534. Mice xenografts injected with human pancreatic cells were used to 
detect the status of tumor growth. The results were: 1) In PANC1 cells, silencing of circ_0007534 attenuated cell proliferation, migration, and invasive ability but promoted cell apoptosis. 2) Circ_0007534 was upregulated in PDAC patients' tissues (undergoing radical surgery) and cancer cells. 3) Circ_0007534 could inhibit cell apoptosis partly via a Bcl-2/caspase- 3 pathway (see details of this pathway in Figure 3 of [3]), noting that the Bax protein is a member of the Bcl-2 family. 4) Based on the xenograft study, it was found that miR - 625 and miR 892b were sponged by circ_0007534. The results of this investigation suggest that anti-sense of circ_0007534 is a potent therapeutic treatment; mimics of either one (or a combination) of miR-626, miR-892b are also plausible anti-PDAC progression measures.

\subsection{The Potential Benefits of microRNA 205 Mimics and Inhibitors of the Long Non-Coding RNA ADPGK-AS1 in Treating Pancreatic Cancers}

Aberrant expressions of miRNAs can induce carcinogenesis by enhancing the function of proto-oncogenes or inhibiting the tumor-suppressing genes (such as TP53). LncRNA can affect the cellular functions at three levels: 1) transcription; 2) post-transcription; 3) epigenetic-meaning the influence arises from non-genetic influences on gene expression [261]. Note also that lncRNA can function as competitive endogenous RNA interacting with encoding genes as well as non-coding miRNA.

The Zinc finger E-box binding homeobox 1 (ZEB1) is a transcription factor involved in cell differentiation and tissue development [262]. ZEB1 is also called E-cadherin transcriptional repressor. Intuitively, without adhesion proteins like E-cadherin, cells are detached, breaking the normal morphology of an organ. On the other hand, it has been found over ten years ago [263] that all five members of the microRNA-200 family (miR-200a, miR-200b, miR-200c, miR-205 miR-141, and miR-429) were markedly downregulated in cells that had undergone EMT in response to transforming growth factor (TGF)- $\beta$. Together, these microRNAs cooperatively regulate expression of the E-cadherin transcriptional repressors ZEB1 and ZEB2, factors previously implicated in EMT and tumor metastasis. Inhibition of the stated five microRNAs was shown to induce EMT in a process accompanied by upregulation of ZEB1 and/or ZEB2. More recently, the issue that ZEB1 could drive the EMT process has also been revisited and confirmed [264].

With such background, in the study of [265], an analysis of the interaction among non-coding RNA ADPGK-AS1, miR205, ZEB1 was carried out with in vitro and in vivo experimentation. Normal pancreatic ductal epithelial line HPDEC, human pancreatic cancer cell lines PANC-1, SW-1990, and embryonic kidney HEK-293T were employed. In the in vivo experiment, human pancreatic cells transfected with 1) miR-205-5p mimics, or 2) ADPGK-AS1 were injected in the mice models. The development of cancer was investigated in real-time. The findings of their project were a) ADPGK-AS1 and ZEB1 were upregulated in the 
cancer cells, leading to the promotion of migration and invasion. b) ADPGK-AS1 was found to target miR-205-5p directly, as ceRNA (competing endogenous RNA) to promote the expression of ZEB. c) ADPGK-AS1 was found to suppress apoptosis. d) In vivo experimental result indicated that the average tumor weight is minimum when treated with miR-205 mimics. This weight became heavier when treated with a mixture of ADPGK-AS1 and miR-205 mimics. The average tumor weight became heaviest when treated with ADPGK-AS1. Mimics of antisense lncRNA ADPGK-AS1 as well as mimics of miRNA-205 are desirable for further preclinical trials.

\subsection{Detection of Exosomes Containing miR-196-3p and miR-204-3p as Markers of Intraductal Papillary Mucinous Neoplasms, Mucinous Cystic Neoplasms, and Pancreatic Cancer}

In the study of [266], exosomes were isolated from four cell lines 1) BxPC-3, 2) Capan-2, 3) PANC-1, 4) MIA PaPe-2. Exosomes (containing some micro mRNAs, called exo-mRNA) were also isolated from sera of 16 patients undergoing pancreatectomy. The tissues of their pancreas were kept for analysis. Based on levels of E-cadherin and vimentin, cells in lines 1) and 2) were found to be the epithelial type, whereas cells in 3) and 4) were of the mesenchymal type. Applying a genome-wide analysis of the exo-mRNA, miR-196b-3p, and miR-636 were identified as downregulated in the epithelial cells, whereas miR-204-3p, miR-3648, \& miR-1497 were identified to be downregulated in mesenchymal type cells. Using the resected tissues and sera from patients with diagnosed pathology, a further analysis showed that miR-196-3p and miR-204-3p were differentially expressed in exosomes of intraductal papillary mucinous neoplasms (IPMN), mucinous cystic neoplasms (MCN), and pancreatic cancer (PC). The exosomes containing these two micro-RNAs may then be taken as markers of the three stages of the pathogenesis of EMT towards pancreatic cancer.

\subsection{Inhibitors of IncRNA SNHG14 and IncMALAT-1 Which Targets the Protein EZH2 That Inhibits the Synthesis of Adhesion Protein E-Cadherin}

In the investigation of [267] on the effects of the long non-coding RNA SNHG14, 58 pairs of pancreatic cancer tissues and adjacent normal tissues from patients undergoing surgical resection were obtained and measured by qRT-PCR. Quantities representing the capacities of cell proliferation and cell invasion were determined. Four human PDAC cell lines Panc1, Panc28, AsPC1, BxPC3, and a human pancreatic ductal epithelial cell line HPDE were used for in vitro studies. The main results were: 1) lncRNA SNHG14 expression was significantly upregulated in PDAC tissues. 2) lncRNA SNHG14 expression was associated with positive lymph node metastasis in patients (Table 1 of [267]). 3) lncRNA SNHG14 expression was also higher in the stated four PDAC cell lines compared 
to that in the HPDE. 4) lncRNA SNHG14 was knocked down by transfection of Panc1 \& Panc28 cells with interference RNAs i.e. si-SNHG14-1 and si-SNHG14-2. There was a significant increase of E-cadherin expression (implying cells are attached, tending to form an organ as normal), but a decrease of vimentin expression in the lncRNA SNHG14 knockdown cells. Since vimentin is an intermediate filament protein and the major cytoskeletal component of mesenchymal cells, low expression of vimentin implies the cells are progressing along anti-EMT direction. 5) Note that Enhancer of zeste homolog 2 (EZH2) is an essential component of the polycomb repressive complex 2 (PRC2), Remark that PRC2 has a role in X chromosome inactivation, in the maintenance of stem cell fate [268]. Aberrant expression of PRC2 has been observed in cancers [269]. There was evidence that EZH2 was recruited to the E-cadherin gene promotor by lncRN MALAT-1 in the study of 2016 [270]. In [267], there was further evidence that another long non-coding RNA SNHG14 would also interact with EZH2, in addition to the effects mentioned in 1) - 4). IncRNA SNHG14 and lncMALAT-1 (presumably detectable in body fluids) are early markers of pancreatic cancer progression, and antisense mimics of these two long non-coding RNAs can be considered as therapeutic measures of the disease.

\section{Concluding Remarks}

1) The proteins encoded by genes, in general, are members participating in signaling pathways involved in many physiological processes, including proliferation, differentiation, apoptosis, cell migration. During embryonic development, organ homeostasis and wound healing processes, cells migrate and collagen and proteins in the fascia are degraded and replaced; new blood vessels are grown; cells die and multiply. In healthy states, all these signaling pathways are regulated in the right time scale and right levels. Off-balance of some signaling pathways would lead to pathological states that are beyond homeostasis and repair. Mutations of the genes related to members of these pathways are the common causes of carcinogenesis.

2) The four major mutations in pancreatic cancers are aberrant activation of the gene KRAS, loss of functions of CDKN2A, SMAD4, TP53. The functions of the signaling pathways associated with such proteins encoded by these genes are discussed in Sections $(3,4,5,6$, and 7$)$. One such protein, e.g. p53, can be related to more than one pathway. There are 8 pathways in Sections (3-5) alone. Moreover, disorders in other signaling pathways can lead to the progression of the disease-from inflammation, via different stages of endothelial-mesenchymal transition (Section (15)). More than ten pathways have been effectively discussed in order to understand the pathology and look for detection and treatment. Apart from the pathways related to the four frequently mutated genes mentioned above, other pathways discussed in this paper include the Hippo (Section (6)), Jak-STAT (Section (8)), NF- $\kappa B$ (Section (9)), Hedgehog (Section (10)), Notch (Section (11)), There can be cross-talks among proteins in differ- 
ent pathways. All these 13 pathways can participate in pancreatic carcinogenesis.

3) As a mixed exocrine-autocrine organ, the pancreas is built of cells of different nature. During development, the ductal cells change their phenotype when they migrate to the duodenum and become the endothelial cells of the digestive system. Inherently, there are i) stem-like cells and ii) stellate cells in the organ. The high occurrence frequency of distant relapse following successful surgery suggests there is spread of cancer cells prior to surgery. We have discussed the early invasion of such cancer stem cells (cells i)) in Sections $(14,17)$. The metastasis of acinar type of ductal cancer cells is summarized in Figure 17. The invasion mechanism of PSCs and cancer cells is via invandopodium (Section (16) with Figure 16).

4) The stellate cells (PSC) play key pathological roles in the progression of tumorigenesis. They secrete the cell membrane-anchored digestive enzyme to degrade the collagen in the basal membrane of the pancreas and connective tissues surrounding, leaving room for cancer cells to migrate. PSCs synthesize excessive collagen as if doing a wound healing work; such "scarring" produces one main part of the stroma. PSCs also secrete abundant hyaluronan which attracts water to become a viscous interstitial fluid with high fluid pressure-this fluid together with the dense collagen fibrils is the main cause of drug resistance (Figure 17). The existence of the newly discovered calcium channel Piezo in PSC, acinar, islet cells strengthen the vicious cycle action initiated by PSCs and their cross-talks with the cancer cells of the organ.

5) The discovery of many non-coding micro RNA or their inhibitors that target the proteins or genes of the pathological pathways and advent in the delivery of pharmaceutical drugs (including such future RNA mimics) by nanoparticles gives us hope to deliver the treatment agents to the tumor site. We have discussed only a small portion of such discovery in the past decade or so.

6) The detection discovery of non-coding RNA, particularly the "sponge micro RNAs" enclosed within vesicles in body fluids is of prime importance. During the past several years, exosomal and non-exosomal micro RNAs have been detected body fluids such as i) blood [271] [272]; ii) saliva [273]; iii) cerebrospinal fluid [253]; iv) urine [274], and v) bile [275].

7) In the future, it is important to comply a database on the interaction characteristics between the micro RNAs (contained in exosomes detected in body fluids) and other RNAs of the healthy normal and that of the patients. Such information is not only useful for designing therapeutic treatments to target the relevant pathways at different stages, for pancreatic cancers, but is also useful for other diseases.

8) Following the above analysis and remarks, we can now state that manifestation of successive pathological states of pancreatic carcinogenesis can be found in compartments of the largest organ of the human body-the fascia. The following table (Table 1) gives a non-exclusive summary of some used drugs, 
potential chemicals, vitamins, micro RNA mimics as early detection of the disease in body fluids and plausible therapeutic treatments of pancreatic cancers.

Table 1. Potential therapeutic agents for treatment or early detection of pancreatic cancers.

\begin{tabular}{|c|c|c|}
\hline $\begin{array}{l}\text { Drugs, chemicals, non-coding RNAs or their } \\
\text { inhibitors as potential treatments, or as diagnostic } \\
\text { markers }\end{array}$ & Proposed Basic action in brief & $\begin{array}{l}\text { Sections in paper and } \\
\text { related figures }\end{array}$ \\
\hline $\begin{array}{l}\text { Irisin (synthetic compound of this hormone has been } \\
\text { achieved in small amount) }\end{array}$ & Target the AMPK-mTOR1 pathway & $(3.3)$ \\
\hline Gemcitabine and nimotuzumab & As monoclonal antibody of epidermal growth factor & $(4.5)$ \\
\hline Phenols, sulfonamides, and their analogs & Target Ras protein in Ras-Raf-Erk pathway & $(4.5)$ \\
\hline Abemaciclib & $\begin{array}{l}\text { To treat hormone receptor-positive, human epidermal } \\
\text { growth factor receptor } 2 \text {-negative breast cancer; drug also } \\
\text { applied to treat pancreatic cancers }\end{array}$ & $(5.2)$ \\
\hline $\begin{array}{l}\mathrm{DD} 44 \mathrm{mT} \text { (di-2-pyridylketone } \\
\text { 4,4-dimthyl-3-thiosemicarbazone), Fe chelator }\end{array}$ & $\begin{array}{l}\text { Activates protein Ndrg1 and hence cyclic-dependent kinase } \\
\text { inhibitor p } 21\end{array}$ & $\begin{array}{l}\text { (5.3), Figure 7, } \\
\text { Figure } 9\end{array}$ \\
\hline DFO (desferrioxamine), Fe chelator & As above & As above \\
\hline $\begin{array}{l}311 \text { (2-hydroxy-1-naphthylal dehydro-isonicotinoyl } \\
\text { hydroazone) }\end{array}$ & As above & As above \\
\hline $\begin{array}{l}\text { RC68, a humanized anti-EGFR monoclonal antibody, } \\
\text { as an antibody-drug conjugate }\end{array}$ & $\begin{array}{l}\text { A monoclonal antibody chemically linked to a drug that is } \\
\text { toxic when the ingredient is released inside the target cell. }\end{array}$ & $\begin{array}{l}\text { (8.5), Figure 7, } \\
\text { Figure } 9\end{array}$ \\
\hline APX3330, compound & $\begin{array}{l}\text { Regulate activities of hypoxia-inducible factor } 1 \alpha(\text { HIF-1 } \alpha) \text {, } \\
\text { NF- } \mathrm{kB} \text {, and function of Jak-STAT3 pathway }\end{array}$ & $\begin{array}{l}\text { (9.4), Figure 2, } \\
\text { Figure 7, Figure } 10\end{array}$ \\
\hline SLC-0111, compound & As above & $(9.4)$ \\
\hline $\begin{array}{l}\text { Combination of cyclopamine and paclitaxel delivered by } \\
\text { nan-particles }\end{array}$ & As an inhibitor of the Hedgehog pathway & (10.3), Figure 11 \\
\hline RO4929097, compound & As an inhibitor of $\gamma$-secretase in Notch pathway & (3.2), Figure 12 \\
\hline ATRA (Vitamin A) & Attenuate endothelial-mesenchymal transition of stellate cell & (18.1), Figure 13 \\
\hline Tocotrienols $\beta, \gamma, \delta$ (sub-family of vitamin E) & Suppress NF- $k B$ mediated inflammation & (18.2), Figure 10 \\
\hline Calcipotriol $(\mathrm{Ca})$, Vitamin D receptor ligand & Reduce inflammation and fibrosis & $(18.3)$ \\
\hline $\begin{array}{l}\text { PEGPH20 (Pegvorhyaluronidase alfa) together } \\
\text { with other anti-cancer drugs }\end{array}$ & Reduce the concentration of interstitial fluid in tumor stroma & (18.4), Figure 17 \\
\hline Micro RNA 96 mimics & Target Ras-Raf-Mek-Erk pathway & (19.2), Figure 3 \\
\hline Micro RNA 34 mimics & $\begin{array}{l}\text { Target Notch pathway and target anti-apoptotic Bcl-2 } \\
\text { protein }\end{array}$ & (19.3), Figure 12 \\
\hline Micro RNAs 21 plus 221 mimics & Treat metastatic pancreatic cancer cells in general & $(19.4)$ \\
\hline Micro RNA 146a mimics & $\begin{array}{l}\text { Downregulates EGFR, downregulates NF- } \kappa B \text {, upregulates } \\
\text { PTEN }\end{array}$ & $\begin{array}{l}\text { (19.5), Figure 2, } \\
\text { Figure 7, Figure 9, } \\
\text { Figure } 10\end{array}$ \\
\hline Antisense non-coding circular RNA IARS mimics & $\begin{array}{l}\text { Reducing endothelial permeability to attenuate cancer } \\
\text { invasion }\end{array}$ & $(20.2)$ \\
\hline Antisense non-coding circular RNA LDLRAD3 mimics & Treats pancreatic cancer cells in general & $(20.3)$ \\
\hline Antisense non-coding circular RNA_100782 & Targets IL6-Jak-STAT3 pathway & (20.4), Figure 7 \\
\hline
\end{tabular}




\section{Continued}

Antisense non-coding circular RNA circ_0007534 mimics

Micro non-coding RNA 205 mimics

Antisense long non-coding RNA ADPGK-AS1 mimics

Detection in body fluids the exosomes containing miR-196-3p and miR-204-3p

Detection of long non-coding RNA SNHG14 and MALAT-1

Antisense of long non-coding RNAs SNHG14 and MALAT-1 mimics
Treat PDAC by releasing the inhibition of Bcl-2-Caspase pathway

Targets protein ZEB1 to reduce cancer cells detachment

Promotes function of Micro non-coding RNA 205 directly

as markers of intraductal papillary mucinous neoplasms, mucinous cystic neoplasms, and pancreatic cancer

These RNAs promote the function of protein EZH2 that inhibits synthesis of adhesion protein E-cadherin (condition of metastasis)

Downregulate function of protein $\mathrm{EZH} 2$ which inhibits the synthesis of adhesion protein E-cadherin
(20.5), Figure 3 of [3] noting Bax is a member of Bcl family

(20.6)

\section{Acknowledgements}

The authors wish to express their gratitude to Mr. Benjamin Fung, brother of PCWF, for his unfailing assistance during the preparation of the manuscript. PCWF wishes to dedicate his work of this series of studies to his late parents Mr. Joseph Wah Hei Fung and Mrs. Helen Woi Suet Lau Fung. All diagrams have been hand-painted by author PCWF.

\section{Conflicts of Interest}

The authors declare no conflicts of interest regarding the publication of this paper.

\section{References}

[1] Cicenas, J., Kvederaviciute, K., Meskinyte, I., Meskinte-Kausilien, E., Skeberdyte, A. and Cicenas Jr., J. (2018) KRAS, TP53, CDKN2A, SMAD4, BRCA1, and BRCA2 Mutations in Pancreatic Cancer. Cancers, 9(5), Article 42, 1-8. https://doi.org/10.3390/cancers 9050042

[2] Fung, P.C.W. and Kong, R.K.C. (2019) Manifestation of Pathological States of Numerous Diseases in the Largest Organ of the Human Body: (I) Basics and the Diseases of Tendon. International Journal of Clinical Medicine, 10, 183-249. https://doi.org/10.4236/ijcm.2019.103018

[3] Fung, P.C.W. and Kong, R.K.C. (2017) The Heat Shock Protein Story-From Taking mTORC1,2 and Heat Shock Protein Inhibitors as Therapeutic Measures for Treating Cancers to Development of Cancer Vaccines. Journal of Cancer Therapy, 8, Article ID: 80657. https://doi.org/10.4236/jct.2017.811086

[4] Siegel, R.L., Miller, K.D., Fedewa, S.A., Ahnen, D.J., Meester, R.G.S., Afsaneh Barzi, A. and Jemal, A. (2017) Colorectal Cancer Statistics. Cancer Journal for Clinicians, 67, 7-30. https://doi.org/10.3322/caac. 21387

[5] Rahib, L., Smith, B.D., Aizenberg, R., Rosenzweig, A.B., Fleshman, J.M. and Matrisian, L.M. (2014) Projecting Cancer Incidence and Deaths to 2030: The Unexpected Burden of Thyroid, Liver, and Pancreas Cancers in the United States. Cancer Research, 74, 2913-2921. https://doi.org/10.1158/0008-5472.CAN-14-0155

[6] La Pak, K.M. and Burd, C.E. (2014) The Molecular Balancing Act of p16 ${ }^{\text {INK4a }}$ in 
Cancer and Aging. Molecular Cancer Research, 12, 167-183.

https://doi.org/10.1158/1541-7786.MCR-13-0350

[7] Pallagi, P., Hegyi, P. and Rakonczay Jr., Z. (2015) The Physiology and Pathophysiology of Pancreatic Ductal Secretion. Pancreas, 44, 1211-1233.

https://doi.org/10.1097/MPA.0000000000000421

[8] Romac, J.M.J., Shahid, R.A., Swain, S.M., Vigna, S.R. and Liddle, R.A. (2018) Piezo1 Is a Mechanically Activated Ion Channel and Mediates Pressure Pancreatitis. Nature Communications, 9, Article 1715, 1-10. https://doi.org/10.1038/s41467-018-04194-9

[9] Mrkonjic, S., Destaing, O. and Albigès-Rizo, C. (2017) Mechanotransduction Pulls the Strings of Matrix Degradation at Invadosome. Matrix Biology, 57/58, 190-203. https://doi.org/10.1016/j.matbio.2016.06.007

[10] Kuntze, A., Goetsch, O., Fels, B., Najder, K., Unger, A., Wilhelmi, M., Sargin, S., Schimmelpfennig, S., Neumann, I., Schwab, A. and Pethő, Z. (2020) Protonation of Piezo1 Impairs Cell-Matrix Interactions of Pancreatic Stellate Cells. Frontiers in Physiology, 11, 89. https://doi.org/10.3389/fphys.2020.00089

[11] Blodgett, D.M., Nowosielska, A., Afik, S., Pechhold, S., Cura, A.J., Kennedy, N.J., Kim, S., Kucukura, A., Davis, R.J., Kent, S.C., Greiner, D.L., Garber, M.G., Harlan, D.M. and Diiorio, P. (2015) Novel Observations from Next-Generation RNA Sequencing of Highly Purified Human Adult and Fetal Islet Cell Subsets. Diabetes, 64, 3172-3181. https://doi.org/10.2337/db15-0039

[12] Beger, H.G., Buchler, M.W., Kozarek, R., Lerch, M.M., Neoptolemos, J.P., Warshaw, A.L., Whitcomb, D.C. and Shiratori, K. (2009) The Pancreas: An Integrated Textbook of Basic Science, Medicine, and Surgery. 2nd Edition, John Wiley \& Sons, Hoboken, Chapters 1-5, 1-62.

[13] Clemente, C.D. (2007) Anatomy-A Regional Atlas of the Human Body. 5th Edition, Plate 19, Lippincott Williams \& Wilkins, Los Angeles.

[14] Murtaugh, L.C. and Keefe, M.D. (2015) Regeneration and Repair of the Exocrine Pancreas. Annual Review of Physiology, 77, 229-249. https://doi.org/10.1146/annurev-physiol-021014-071727

[15] Ferdek, P.E. and Jakubowska, M.A. (2017) Biology of Pancreatic Stellate Cells-More than Just Pancreatic Cancer. Pflügers Archiv-European Journal of Physiology, 469, 1039-1050. https://doi.org/10.1007/s00424-017-1968-0

[16] Li, W., Zhou, Y., Wang, X., Cai, M., Gao, F., Per-Ola Carlsson, P.O. and Sun, Z. (2019) A Modified in Vitro Tool for Isolation and Characterization of Rat Quiescent Islet Stellate Cells. Experimental Cell Research, 384, Article ID: 111617. https://doi.org/10.1016/j.yexcr.2019.111617

[17] Vadlakonda, L., Dash, A., Pasupuleti, M., Kumar, K.A. and Reddanna, P. (2013) The Paradox of Akt-mTOR Interactions. Frontiers in Oncology, 3, Article 165. https://doi.org/10.3389/fonc.2013.00165

[18] Bader, A.G., Kang, S., Zhao, L. and Vogt, P.K. (2005) Oncogenic PI3K Deregulates Transcription and Translation. Nature Reviews Cancer, 5, 921-929. https://doi.org/10.1038/nrc1753

[19] Fu, Z. and Tindall, D.J. (2008) FOXOs, Cancer and Regulation of Apoptosis. Oncogene, 27, 2312-2319. https://doi.org/10.1038/onc.2008.24

[20] Karar, J. and Maity, A. (2011) PI3K/AKT/mTOR Pathway in Angiogenesis. Frontiers in Molecular Neuroscience, 4, 51. https://doi.org/10.3389/fnmol.2011.00051

[21] Simpson, L. and Parsons, R. (2001) PTEN: Life as a Tumor Suppressor. Experimen- 
tal Cell Research, 264, 29-41. https://doi.org/10.1006/excr.2000.5130

[22] NCBI EIF4EBP1 Eukaryotic Translation Initiation Factor 4E Binding Protein 1 [Homo sapiens (Human)], Full Report. Gene ID: 1978, Updated on 18 August, 2020.

[23] Qin, X., Jiang, B. and Zhang, Y. (2016) 4E-BP1, a Multifactor Regulated Multifunctional Protein. Cell Cycle, 15, 781-786. https://doi.org/10.1080/15384101.2016.1151581

[24] Huang, J., Dibble, C.C., Matsuzaki, M. and Manning, B.D. (2008) The TSC1-TSC2 Complex Is Required for Proper Activation of mTOR Complex 2. Molecular and Cellular Biology, 28, 4104-4115. https://doi.org/10.1128/MCB.00289-08

[25] Deivasikamani, V., Dhayalan, S., Abudushalamu, Y., Mughal, R., Visnagri, A., Cuthbertson, K., Scragg, J.L., Munsey, T.S., Viswambharan, H., Muraki, K., Foster, R., Sivaprasadarao, A., Kearney, M.T., Beech, D.J. and Piruthivi Sukumar, P. (2019) Piezo1 Channel Activation Mimics High Glucose as a Stimulator of Insulin Release. Scientific Reports, 9, Article ID: 16876. https://doi.org/10.1038/s41598-019-51518-w

[26] Sheu, F.S., Zhu, W. and Fung, P.C.W. (2000) Direct Observation of Trapping and Release of Nitric Oxide by Glutathione and Cysteine with Electron Paramagnetic Resonance Spectroscopy. Biophysical Journal, 78, 1216-1226. https://doi.org/10.1016/S0006-3495(00)76679-3

[27] Hanahan, D. and Folkman, J. (1996) Patterns and Emerging Mechanisms of the Angiogenic Switch during Tumorigenesis. Cell, 86, 353-364.

https://doi.org/10.1016/S0092-8674(00)80108-7

[28] Bostrom, P., Wu, J., Jedrychowski, M.P., Korde, A., Li, Y., Lo, J.C., Rasbach, K.A., Boström, E.A., Choi, J.H., Long, J.Z., Kajimura, S., Zingaretti, M.C., Vind, B.F., Hua, T., Cinti, S., Højlund, K., Gygi, S.P. and Spiegelman, B.M. (2012) A PGC1-Alpha-Dependent Myokine That Drives Brown-Fat-Like Development of White Fat and Thermogenesis. Nature, 481, 463-468.

https://doi.org/10.1038/nature10777

[29] Liu, J., Song, N., Huang, Y. and Chen, Y. (2018) Irisin Inhibits Pancreatic Cancer Cell Growth via the AMPK-mTOR Pathway. Scientific Reports, 8, Article ID: 15247. https://doi.org/10.1038/s41598-018-33229-w

[30] Zhang, D., Zhang, P., Li, L., Tang, N., Huang, F., Kong, X., Tan, X. and Shi, G. (2019) Irisin Functions to Inhibit Malignant Growth of Human Pancreatic Cancer Cells via Downregulation of the PI3K/AKT Signaling Pathway. OncoTargets and Therapy, 12, 7243-7249. https://doi.org/10.2147/OTT.S214260

[31] Zhang, D., Tan, X., Tang, N., Huang, F., Chen, Z. and Shi, G. (2020) Review of Research on the Role of Irisin in Tumors. OncoTargets and Therapy, 13, 4423-4430. https://doi.org/10.2147/OTT.S245178

[32] Wucherpfennig, T.G., Muller, S., Wolfrum, C. and Bode, J.W. (2016) Chemical Synthesis of the $12 \mathrm{kDa}$ Human Myokine Irisin by $\alpha$-Ketoacid-Hydroxylamine (KAHA) Ligation. Helvetica Chimica Acta, 99, 897-907. https://doi.org/10.1002/hlca.201600160

[33] Lad, Y., McHugh, B., Hodkinson, P.S., MacKinnon, A.C., Haslett, C., Ginsberg, M.H. and Sethi, T. (2006) PLCe Suppresses Integrin Activation. The Journal of Biological Chemistry, 281, 29501-29512. https://doi.org/10.1074/jbc.M513471200

[34] Cullen, P.J. and Lockyer, P.J. (2002) Integration of Calcium and Ras Signalling. Nature Reviews Molecular Cell Biology, 3, 339-348. https://doi.org/10.1038/nrm808

[35] Mebratu, Y. and Tesfaigzi, Y. (2009) How ERK1/2 Activation Controls Cell Prolife- 
ration and Cell Death: Is Subcellular Localization the Answer? Cell Cycle, 8, 1168-1175. https://doi.org/10.4161/cc.8.8.8147

[36] Yuan, P., He, X.H., Rong, Y.F., Cao, J., Li, Y., Hu, Y.P., Liu, Y., Li, D., Lou, W. and Liu, M.F. (2017) KRAS/NF-kB/YY1/miR-489 Signaling Axis Controls Pancreatic Cancer Metastasis. Cancer Research, 77, 100-111. https://doi.org/10.1158/0008-5472.CAN-16-1898

[37] Fung, P.C.W. (2013) Chapter 5. Plausible Biomedical Consequences of Acupuncture Applied at Sites Characteristic of Acupoints in the Connective-Tissue-Interstitial-Fluid System. In: Chen, L.L. and Cheng, T.O., Eds., Acupuncture in Modern Medicine, InTech Open, Rijeka, 95-131.

[38] Liu, C.Y., Lin, H.H., Tang, M.J. and Wang, Y.K. (2015) Vimentin Contributes to Epithelial-Mesenchymal Transition Cancer Cell Mechanics by Mediating Cytoskeletal Organization and Focal Adhesion Maturation. Oncotarget, 6, 15966-15983. https://doi.org/10.18632/oncotarget.3862

[39] Coste, B., Mathur, J., Schmidt, M., Earley, T.J., Ranade, S., Petrus, M.J., Dubin, A.E. and Patapoutian, A. (2010) Piezo1 and Piezo2 Are Essential Components of Distinct Mechanically-Activated Cation Channels. Science, 330, 55-60.

https://doi.org/10.1126/science.1193270

[40] Ellefsen, K.L., Holt, J.R., Chang, A.C., Nourse, J.L., Arulmoli, J., Mekhdjian, A.H., Abuwarda, H., Tombola, F., Flanagan, L.A., Dunn, A.R., Parker, I., Medha, M. and Pathak, M.M. (2019) Myosin-II Mediated Traction Forces Evoke Localized Piezo1-Dependent $\mathrm{Ca}^{2+}$ Flickers. Scientific Report. Communications Biology, 2, 298. https://doi.org/10.1038/s42003-019-0514-3

[41] DuFort, C.C., DelGiorno, K.E., Carlson, M.A., Osgood, R.J., Zhao, C., Huang, Z., Thompson, C.B., Connor, R.J., Thanos, C.D., Brockenbrough, J.S., Provenzano, P.P., Frost, G.I., Shepard, H.M. and Hingorani, S.R. (2016) Interstitial Pressure in Pancreatic Ductal Adenocarcinoma Is Dominated by a Gel-Fluid Phase. Biophysical Journal, 110, 2106-2119. https://doi.org/10.1016/j.bpj.2016.03.040

[42] Pedersen, S.F., Novak, I., Alves, F., Schwab, A. and Pardo, L.A. (2017) Alternating pH Landscapes Shape Epithelial Cancer Initiation and Progression: Focus on Pancreatic Cancer. Bioessays, 39, Article ID: 1600253.

https://doi.org/10.1002/bies.201600253

[43] Fels, B., Nielsen, N. and Schwab, A. (2016) Role of TRPC1 Channels in Pressure-Mediated Activation of Murine Pancreatic Stellate Cells. European Biophysics Journal, 2016, 1-14. https://doi.org/10.1155/2016/7406356

[44] Lau, K.S. and Haigis, K.M. (2009) Non-Redundancy within the RAS Oncogene Family: Insights into Mutational Disparities in Cancer. Molecules and Cells, 28, 315-320. https://doi.org/10.1007/s10059-009-0143-7

[45] Sun, Q., Burke, J.P., Phan, J., Burns, M.C., Olejniczak, E.T., Waterson, A.G., Lee, T., Rossanese, O.W. and Fesik, S.W. (2012) Discovery of Small Molecules that Bind to K-Ras and Inhibit Sos-Mediated Activation. Angewandte Chemie International Edition in English, 51, 6140-6143. https://doi.org/10.1002/anie.201201358

[46] Schultheis, B., Reuter, D., Ebert, M.P., Siveke, J., Kerkhoff, A., Berdel, W.E., Hofheinz, R., Behringer, D.M., Schmidt, W.E., Goker, E., Dosso, S.D., Kneba, M., Yalcin, S., Overkamp, F., Schlegel, F., Dommach, M., Rohrberg, R., Steinmetz, T. and Strumberg, D. (2017) Gemcitabine Combined with the Monoclonal Antibody Nimotuzumab Is an Active First-Line Regimen in KRAS Wildtype Patients with Locally Advanced or Metastatic Pancreatic Cancer: A Multicenter, Randomized Phase IIb Study. Annals of Oncology, 28, 2429-2435. 
https://doi.org/10.1093/annonc/mdx343

[47] Schutte, M., Hruban, R.H., Geradts, J., Maynard, R., Hilgers, W., Rabindran, S.K., Moskaluk, C.A., Hahn, S.A., Schwarte-Waldhoff, I., Schmiegel, W., Baylin, S.B., Kern, S.E. and Herman, J.G. (1997) Abrogation of the RB/p16 Tumor-Suppressive Pathway in Virtually All Pancreatic Carcinomas. Cancer Research, 57, 3126-3130.

[48] Wood, L.D. and Hruban, R.H. (2012) Pathology and Molecular Genetics of Pancreatic Neoplasms. Journal of Cancer, 18, 492-501. https://doi.org/10.1097/PPO.0b013e31827459b6

[49] LaPak, K.M. and Burd, C.E. (2014) The Molecular Balancing Act of p16 $6^{\text {INK4a }}$ in Cancer and Aging. Molecular Cancer Research, 12, 167-183.

https://doi.org/10.1158/1541-7786.MCR-13-0350

[50] Xiong, Y., Hannon, G., Zhang, H., Casso, D. and Beach, D. (1993) p21 Is a Universal Inhibitor of Cyclin Kinases. Nature, 366, 701-704. https://doi.org/10.1038/366701a0

[51] Duronio, R.J. and Xiong, Y. (2013) Signaling Pathways that Control Cell Proliferation. Cold Spring Harbor Perspectives in Biology, 5, Article ID: a008904. https://doi.org/10.1101/cshperspect.a008904

[52] Tang, B., Li, Y., Yuan, S., Wang, Z., Yu, S., Li, B. and He, S. (2015) Clinicopathological Significance of CDKN2A Promoter Hypermethylation Frequency with Pancreatic Cancer. Scientific Reports, I5, Article ID: 13563I. https://doi.org/10.1038/srep13563

[53] Kim, E.S. (2017) Abemaciclib: First Global Approval. Drugs, 77, 2063-2070. https://doi.org/10.1007/s40265-017-0840-Z

[54] Schettini, F., De Santo, I., Rea, C.G., De Placido, P., Formisano, L., Giuliano, M., Arpino, G., De Laurentiis, M., Puglisi, F., De Placido, S. and Del Mastro, L. (2018) CDK 4/6 Inhibitors as Single Agent in Advanced Solid Tumors. Frontiers in Oncology, 8, Article 608, 1-12. https://doi.org/10.3389/fonc.2018.00608

[55] Le, N.T. and Richardson, D.R. (2004) Iron Chelators with High Antiproliferative Activity Up-Regulate the Expression of Growth Inhibitory and Metastasis Suppressor Gene: A Link between Iron Metabolism and Proliferation. Blood, 104, 2967-2975. https://doi.org/10.1182/blood-2004-05-1866

[56] Zhang, S., Yu, C., Yang, X., Lu, J., Hu, W., Hao, X., Li, S., Aikemu, B., Yang, G., He, Z., Zhang, L., Xue, P., Cai, Z., Ma, J., Zang, L., Feng, B., Yuan, F., Sun, J. and Zheng, M. (2019) N-myc Downstream-Regulated Gene 1 Inhibits the Proliferation of Colorectal Cancer through Emulative Antagonizing NEDD4-Mediated Ubiquitylation of p21. Journal of Experimental \& Clinical Cancer Research, 38, 490. https://doi.org/10.1186/s13046-019-1476-5

[57] Kurdistani, S.K., Arizti, P., Reimer, C.L., Sugrue, M.M., Aaronson, S.A. and Lee, S.W. (1998) Inhibition of Tumor Cell Growth by RTP/rit42 and Its Responsiveness to p53 and DNA Damage. Cancer Research, 58, 4439-4444.

[58] Stein, S., Thomas, E.K., Herzog, B., Westfall, M.D., Rocheleau, J.V., Jackson, R.S., Wang, M. and Liang, P. (2004) NDRG1 Is Necessary for p53-Dependent Apoptosis. The Journal of Biological Chemistry, 279, 48930-48940.

https://doi.org/10.1074/jbc.M400386200

[59] Maruyama, Y., Ono, M., Kawahara, A., Yokoyama, T., Basaki, Y., Kage, M., Aoyagi, S., Kinoshita, H. and Kuwano, M. (2006) Tumor Growth Suppression in Pancreatic Cancer by a Putative Metastasis Suppressor Gene Cap43/NDRG1/Drg-1 through Modulation of Angiogenesis. Cancer Research, 66, 6232-6242. 
https://doi.org/10.1158/0008-5472.CAN-06-0183

[60] Whitnall, M., Howard, J., Ponka, P. and Richardson, D.R. (2006) A Class of Iron Chelators with a Wide Spectrum of Potent Antitumor Activity that Overcomes Resistance to Chemotherapeutics. Proceedings of the National Academy of Sciences of the United States of America, 103, 14901-14906.

https://doi.org/10.1073/pnas.0604979103

[61] Kovacevic, Z., Chikhani, S., Lovejoy, D.B. and Richardson, D.R. (2011) Novel Thiosemicarbazone Iron Chelators Induce Up-Regulation and Phosphorylation of the Metastasis Suppressor, NDRG1: A New Strategy for the Treatment of Pancreatic Cancer. Molecular Pharmacology, 80, 598-609. https://doi.org/10.1124/mol.111.073627

[62] Varelas, X. (2014) The Hippo Pathway Effectors TAZ and YAP in Development, Homeostasis, and Diseases. Development, 141, 1614-1626.

https://doi.org/10.1242/dev.102376

[63] Zhao, B., Ye, X., Yu, J.D., Li, L., Li, W.Q., Li, S.M., Yu, J.J., Lin, J., Wang, C.Y., Chinnaiyan, A.M., Lai, Z.C. and Guan, K.L. (2008) TEAD Mediates YAP-Dependent Gene Induction and Growth Control. Genes \& Development, 22, 1962-1971. https://doi.org/10.1101/gad.1664408

[64] Jiao, S., Wang, H.Z., Shi, Z.B., Zhang, W.J., Song, X.M., He, F., Wang, Y.C., Zhang, Z.Z., Wang, X., Guo, T., Li, P.X., Zhao, Y., Ji, H.B., Zhang, L. and Zhou, Z.C. (2014) A Peptide Mimicking VGLL4 Function Acts as a YAP Antagonist Therapy against Gastric Cancer. Cancer Cell, 25, 166-180. https://doi.org/10.1016/j.ccr.2014.01.010

[65] Wu, D.M., Shan Wang, S., Wen, X., Han, X.H., Wang, Y.J., Shen, M., Fan, S.H., Zhang, Z.F., Shan, Q., Li, M.Q., Hu, B., Lu, J., Chen, G.Q. and Zheng, Y.L. (2018) LncRNA SNHG15 Acts as a ceRNA to Regulate YAP1-Hippo Signaling Pathway by Sponging miR-200a-3p in Papillary Thyroid Carcinoma. Cell Death and Disease, 9, 947. https://doi.org/10.1038/s41419-018-0975-1

[66] PTPN14 Protein Tyrosine Phosphatase Non-Receptor Type 14 [Homo sapiens (Human)], Gene ID: 5784, NCBI Full Report, Updated on 5-Jul-2020.

[67] Raj, N. and Attardi, L.D. (2017) The Transactivation Domains of the p53 Protein. Cold Spring Harbor Perspectives in Biology, 7, Article ID: a026047.

https://doi.org/10.1101/cshperspect.a026047

[68] Mello, S.S., Valente, L.J., Raj, N., Seoane, J.A., Flowers, B.M., McClendon, J., Bieging-Rolett, K.T., Lee, J., Ivanochko, D., Kozak, M.M., Chang, D.T., Longacre, T.A., Koong, A.C., Arrowsmith, C.H., Kim, S.K., Vogel, H., Wood, L.D., Hruban, R.H., Curtis, C. and Attardi, L.D. (2017) A p53 Super-Tumor Suppressor Reveals a Tumor Suppressive p53-Otpn14-Yap Axis in Pancreatic Cancer. Cancer Cell, 32, 460-473. https://doi.org/10.1016/j.ccell.2017.09.007

[69] Brady, C.A., Jiang, D., Mello, S.S., Johnson, T.M., Jarvis, L.A., Kozak, M.M., Broz, D.K., Basak, S., Park, E.J., McLaughlin, M.E., Karnezis, A.N. and Attardi, L.D. (2011) Distinct p53 Transcriptional Programs Dictate Acute DNA-damage Response and Tumor Suppression. Cell, 145, 571-583. https://doi.org/10.1016/j.cell.2011.03.035

[70] Bruan, E. and Sauter, D. (2019) Furin-Mediated Protein Processing in Infectious Diseases and Cancer. Clinical \& Translational Immunology, 2019, Article ID: e1073. https://doi.org/10.1002/cti2.1073

[71] Zhang, Y., Zhou, M., Wei, H., Zhou, H., He, J., Lu, Y., Wang, D., Chen, B. Zeng, J., Peng, W., Du, F., Gong, A. and $\mathrm{Xu}$, M. (2017) Furin Promotes Epithelial-Mesenchymal Transition in Pancreatic Cancer Cells in Hippo-Yap Pathway. In- 
ternational Journal of Oncology, 50, 1352-1362.

https://doi.org/10.3892/ijo.2017.3896

[72] Meng, Z., Moroishi, T. and Guan, K.L. (2016) Mechanisms of Hippo Pathway Regulation. Genes \& Development, 30, 1-17.

https://doi.org/10.1101/gad.274027.115

[73] UniProtKB-Q8IX03 (KIBRA_HUMAN).

[74] Becker, G.L., Hardes, K. and Steinmetzer, T. (2011) New Substrate Analogue Furin Inhibitors Derived from 4-Amidinobenzylamide. Bioorganic \& Medicinal Chemistry Letters, 21, 4695-4697. https://doi.org/10.1016/j.bmcl.2011.06.091

[75] Lanuza-Masdeu, J., Arévalo, M.I., Vila, C., Albert Barberà, A., Gomis, R. and Caelles, C. (2013) In Vivo JNK Activation in Pancreatic b-Cells Leads to Glucose Intolerance Caused by Insulin Resistance in Pancreas. Diabetes, 62, 2308-2317. https://doi.org/10.2337/db12-1097

[76] Codelia, V.A., Sun, G. and Irvine, K.D. (2014) Regulation of YAP by Mechanical Strain through Jnk and Hippo Signaling. Current Biology, 24, 2012-2017. https://doi.org/10.1016/j.cub.2014.07.034

[77] Wang, Y., Solt, L.A., Kojetin, D.J. and Burris, T.P. (2012) Regulation of p53 Stability and Apoptosis by a ROR Agonist. PLoS ONE, 7, e34921. https://doi.org/10.1371/journal.pone.0034921

[78] Su, J., Bo Su, B., Xia, H., Liu, F., Zhao, X.H., Li, J., Zhang, J.Z., Shi, Y., Zeng, Y., Zeng, X., Ling, H., Wu, Y.H. and Su, Q. (2019) ROR $\alpha$ Suppresses Epithelial-to-Mesenchymal Transition and Invasion in Human Gastric Cancer Cells via the Wnt/ $\beta$-Catenin Pathway. Frontiers in Oncology, 9, 1344. https://doi.org/10.3389/fonc.2019.01344

[79] Yu, F.X., Zhao, B., Panupinthu, N., Jewell, J.L., Lian, I., Wang, L.H., Zhao, J.G., Yuan, H.X., Tumaneng, K., Li, H., Fu, X.D., Mills, G.M. and Guan, K.L. (2012) Regulation of the Hippo-YAP Pathway by G-Protein-Coupled Receptor Signaling. Cell, 150, 669-670. https://doi.org/10.1016/j.cell.2012.06.037

[80] Philippe, C., Pinson, B., Dompierre, J., Pantesco, V., Viollet, B., Daignan-Fornier, B. and Moenner, M. (2018) AICAR Antiproliferative Properties Involve the AMPK-Independent Activation of the Tumor Suppressors LATS 1 and 2. Neoplasia, 6, 555-562. https://doi.org/10.1016/j.neo.2018.03.006

[81] Corton, J.M., Gillespie, J.G., Hawley, S.A. and Hardie, D.G. (1995) 5-Aminoimidazole-4-Carboxamide Ribonucleoside. A Specific Method for Activating AMP-Activated Protein Kinase in Intact Cells? European Journal of Biochemistry, 229, 558-565. https://doi.org/10.1111/j.1432-1033.1995.tb20498.x

[82] Zucker, S., Cao, J. and Chen, W.T. (2000). Critical Appraisal of the Use of Matrix Metalloproteinase Inhibitors in Cancer Treatment. Oncogene, 19, 6642-6650. https://doi.org/10.1038/sj.onc.1204097

[83] Coppola, J.M., Mahaveer, S., Bhojani, M.S., Ross, B.D. and Rehemtulla, A. (2008) A Small-Molecule Furin Inhibitor Inhibits Cancer Cell Motility and Invasiveness. Neoplasia, 10, 363-370. https://doi.org/10.1593/neo.08166

[84] Massagué, J. (1998) TGF- $\beta$ Signal Transduction. Annual Review of Biochemistry, 67, 753-791. https://doi.org/10.1146/annurev.biochem.67.1.753

[85] Shi, Y. and Massagué, J. (2003) Mechanisms of TGF- $\beta$ Signaling from Cell Membrane to the Nucleus. Cell, 113, 685-700.

https://doi.org/10.1016/S0092-8674(03)00432-X

[86] Tang, W.B., Ling, G.H., Lin Sun, L. and Liu, F.Y. (2010) Smad Anchor for Receptor 
Activation (SARA) in TGF-Beta Signaling. Frontiers in Bioscience, 2, 857-860. https://doi.org/10.2741/e147

[87] SMAD2 SMAD Family Member 2 [Homo sapiens (Human)], Gene ID: 4087, NCBI Full Report, Updated on 6-Sep-2020.

[88] Levy, L. and Hill, C.S. (2005) Smad4 Dependency Defines Two Classes of Transforming Growth Factor $\beta$ (TGF- $\beta$ ) Target Genes and Distinguishes TGF- $\beta$-Induced Epithelial Mesenchymal Transition from Antiproliferative and Migratory Responses. Molecular and Cellular Biology, 25, 8108-8125. https://doi.org/10.1128/MCB.25.18.8108-8125.2005

[89] Inoue, M., Sawada, T., Uchima, Y. and Kimura, K. (2006) Plasminogen Activator Inhibitor-1 (PAI-1) gene Transfection Inhibits the Liver Metastasis of Pancreatic Cancer by Preventing Angiogenesis. Oncology Reports, 14, 1445-1451.

https://doi.org/10.3892/or.14.6.1445

[90] UniProtKB Q02952 (AKA12_HUMAN).

[91] Hayashi, M., Nomoto, S., Kanda, M., Okamura, Y., Nishikawa, Y., Yamada, S., Fujii, T., Sugimoto, H., Takeda, S. and Kodera, Y. (2012) Identification of the A Kinase Anchor Protein 12 (AKAP12) Gene as a Candidate Tumor Suppressor of Hepatocellular Carcinoma. Journal of Surgical Oncology, 105, 381-386.

https://doi.org/10.1002/jso.22135

[92] Xia, W., Ni, J., Zhuang, J.H., Qiana, L.X., Wang, P. and Wang, J.N. (2016) MiR-103 Regulates Hepatocellular Carcinoma Growth by Targeting AKAP12. The International Journal of Biochemistry \& Cell Biology, 7, 1-11.

https://doi.org/10.1016/j.biocel.2015.11.017

[93] UniProtKB-P18564 (ITB6_HUMAN).

[94] Blackford, A., Serrano, O.K., Wolfgang, C.L., Parmigiani, G., Jones, S., Zhang, X.S., Parsons, D.W., Cheng, J.H.L., Leary, R.J., Eshleman, J.R., Goggins, M., Jaffee, E.M., Iacobuzio-Donahue, C.A., Maitra, A., Cameron, J.L., Olino, K., Schulick, R., Winter, J., Herman, J.M., Laheru, D., Klein, A.P., Vogelstein, B., Kinzler, K.W., Velculescu, V.E. and Hruban, R.H. (2009) SMAD4 Gene Mutations Are Aassociated with Poor Prognosis in Pancreatic Cancer. Clinical Cancer Research, 15, 4674-4679.

https://doi.org/10.1158/1078-0432.CCR-09-0227

[95] Hoshino, Y., Nishida, J., Katsuno, Y., Koinuma, D., Aoki, T., Kokudo, N., Miyazono, K. and Ehata, S. (2015) Smad4 Decreases the Population of Pancreatic Cancer-Initiating Cells through Transcriptional Repression of ALDH1A1. The American Journal of Pathology, 185, 1457-1470.

https://doi.org/10.1016/j.ajpath.2015.01.011

[96] Marcato, P., Dean, C.A., Giacomantonio, C.A. and Lee, P.W. (2011) Aldehyde Dehydrogenase: Its Role as a Can Stem Cell Marker Comes Down to the Specific Isoform. Cell Cycle, 10, 1378-1384. https://doi.org/10.4161/cc.10.9.15486

[97] Henson, E.S. and Gibson, S.B. (2006) Surviving Cell Death through Epidermal Growth Factor (EGF) Signal Transduction Pathways: Implications for Cancer Therapy. Cellular Signalling, 18, 2089-2097.

https://doi.org/10.1016/j.cellsig.2006.05.015

[98] Du, Y., Shen, J., Hsu, J.L., Han, Z., Hsu, M.C., Yang, C.C., Kuo, H.P., Wang, Y.N., Yamaguchi, H., Miller, S.A. and Hung, M.C. (2014) Syntaxin 6-Mediated Golgi Translocation Plays an Important Role in Nuclear Functions of EGFR through Microtubule-Dependent Trafficking. Oncogene, 33, 756-770.

https://doi.org/10.1038/onc.2013.1

[99] Veigel, C. and Schmidt, C.F. (2011) Moving into the Cell: Single-Molecule Studies 
of Molecular Motors in Complex Environments. Nature Reviews Molecular Cell Biology, 12, 163-176. https://doi.org/10.1038/nrm3062

[100] Hirokawa, N. (1998) Kinesin and Dynein Superfamily Proteins and the Mechanism of Organelle Transport. Science, 279, 519-526. https://doi.org/10.1126/science.279.5350.519

[101] Wendler, F. and Tooze, S.A. (2001) Syntaxin6: The Promiscuous Behavior of SNARE Protein. Traffic, 2, 606-611. https://doi.org/10.1034/j.1600-0854.2001.20903.x

[102] Springer, S., Spang, A. and Schekman, R. (1999) A Primer on Vesicle Budding. Cell, 97, 145-148. https://doi.org/10.1016/S0092-8674(00)80722-9

[103] Wang, Y.N., Wang, H., Yamaguchi, H., Lee, H.J., Lee, H.H. and Hung, M.C. (2010) COPI-Mediated Retrograde Trafficking from the Golgi to the ER Regulates EGFR Nuclear Transport. Biochemical and Biophysical Research Communications, 399, 498-504. https://doi.org/10.1016/j.bbrc.2010.07.096

[104] Gogala, M., Becker, T., Beatrix, B., Armache, J.P., Barrio-Garcia, C., Berninghausen, O. and Beckmann, R. (2014) Structures of the Sec61 Complex Engaged in Nascent Peptide Translocation or Membrane Insertion. Nature, 506, 107-110. https://doi.org/10.1038/nature12950

[105] Y.N., Lee, H.H., Lee, H.J., Du, Y., Yamaguchi, H. and Hung, M.C. (2012) Membrane-Bound Trafficking Regulates Nuclear Transport of Integral Epidermal Growth Factor Receptor (EGFR) and ErbB-2. The Journal of Biological Chemistry, 287, 16869-16879. https://doi.org/10.1074/jbc.M111.314799

[106] Xiong, H.Q. and Abbruzzese, J.L. (2002) Epidermal Growth Factor Receptor-Targeted Therapy for Pancreatic Cancer. Seminars in Oncology, 29, 31-37. https://doi.org/10.1053/sonc.2002.35645

[107] Brand, T.M., Iida, M., Luthar, N., Star, M.M., Huppert, E.J. and Wheeler, D.L. (2013) Nuclear EGFR as a Molecular Target in Cancer. Radiotherapy \& Oncology, 108, 370-377. https://doi.org/10.1016/j.radonc.2013.06.010

[108] Rafiq, N.B.M., Nishimura, Y., Plotnikov, S.V., Thiagarajan, V., Zhang, Z., Shi, S.D., Natarajan, M., Viasnoff, V., Kanchanawong, P., Jones, G.E. and Bershadsky, A.D. (2019) A Mechano-Signalling Network Linking Microtubules, Myosin IIA Filaments and Integrin-Based Adhesions. Nature Materials, 18, 638-649. https://doi.org/10.1038/s41563-019-0371-y

[109] Lin, S.Y., Makino, K., Xia, W., Matin, A., Wen, Y., Kwong, K.Y., Bourguignon, L. and Hung, M.C. (2001) Nuclear Localization of EGF Receptor and Its Potential New Role as a Transcription Factor. Nature Cell Biology, 3, 802-808. https://doi.org/10.1038/ncb0901-802

[110] Cunningham, D., Humblet, Y., Siena, S., Khayat, D., Bleiberg, H., Santoro, A., Bets, D., Mueser, M., Harstrick, A., Verslype, C., Chau, I. and van Cutsem, E. (2004) Cetuximab Monotherapy and Cetuximab Plus Irinotecan in Irinotecan Refractory Metastatic Colorectal Cancer. New England Journal of Medicine, 351, 337-345. https://doi.org/10.1056/NEJMoa033025

[111] Wu, M., Rivkin, A. and Pham, T. (2008) Panitumumab: Human Monoclonal Antibody against Epidermal Growth Factor for the Treatment of Metastatic Colorectal Cancer. Clinical Therapeutics, 30, 14-30. https://doi.org/10.1016/j.clinthera.2008.01.014

[112] Thatcher, N., Hirsch, F.R., Luft, A.V., Szczesna, A., Ciuleanu, T.E., Dediu, M., Ramlau, R., Galiulin, R.K., Bálint, B., Losonczy, G., Kazarnowicz, A., Park, K., Schumann, C., Reck, M., Depenbrock, H., Nanda, S., Kruljac-Letunic, A., Kurek, R., 
Paz-Ares, L. and Socinski, M.A. (2015) Necitumumab Plus Gemcitabine and Cisplatin versus Gemcitabine and Cisplatin Alone as First-Line Therapy in Patients with Stage IV Squamous Non-Small-Cell Lung Cancer (SQUIRE): An Openlabel, Randomised, Controlled Phase 3 Trial. The Lancet Oncology, 16, 763-774. https://doi.org/10.1016/S1470-2045(15)00021-2

[113] Lu, M., Wang, X.C., Shen, L., Jia, J., Gong, J.F., Li, J., Li, J., Li, Y., Zhang, X.T., Lu, Z.H., Zhou, J. and Zhang, X.Z. (2016) Nimotuzumab Plus Paclitaxel and Cisplatin as the First Line Treatment for Advanced Esophageal Squamous Cell Cancer: A Single Centre Prospective Phase II Trial. Cancer Science, 170, 486-490. https://doi.org/10.1111/cas.12894

[114] Li, Z., Wang, M., Yao, X., Luo, W., Qu, Y., Yu, D., Li, X., Fang, J. and Huang, C. (2019) Development of a Novel EGFR-Targeting Antibody-Drug Conjugate for Pancreatic Cancer Therapy. Targeted Oncology, 14, 93-105.

https://doi.org/10.1007/s11523-018-0616-8

[115] Oeckinghaus, A. and Ghosh, S. (2009) The NF-kB Family of Transcription Factors and Its Regulation. Cold Spring Harbor Perspectives in Biology, 1, Article ID: a000034. https://doi.org/10.1101/cshperspect.a000034

[116] Liu, T., Zhang, L., Joo, D. and Sun, S.C. (2017) NF-кB Signaling in Inflammation. Signal Transduction and Targeted Therapy, 2, Article ID: e17023.

https://doi.org/10.1038/sigtrans.2017.23

[117] Crowe, P.D., VanArsdale, T.L., Walter, B.N., Ware, C.F., Hession, C., Ehrenfels, B., Browning, J.L., Din, W.S., Goodwin, R.G. and Smith, C.A. (1994) A Lymphotoxin-Beta-Specific Receptor. Science, 264, 707-710.

https://doi.org/10.1126/science.8171323

[118] Nedwin, G.E., Naylor, S.L., Sakaguchi, A.Y., Smith, D., Jarrett-Nedwin, J., Pennica, D., Goeddel, D.V. and Gray, P.W. (1985) Human Lymphotoxin and Tumor Necrosis Factor Genes: Structure, Homology and Chromosomal Localization. Nucleic Acids Research, 13, 6361-6373. https://doi.org/10.1093/nar/13.17.6361

[119] Taniguchi, K. and Karin, M. (2018) NF- $\kappa B$, Inflammation, Immunity and Cancer: Coming of Age. Nature Reviews Immunology, 18, 309-324.

https://doi.org/10.1038/nri.2017.142

[120] Frank, P.G. and Lisanti, M.P. (2008) ICAM-1: Role in Inflammation and in the Regulation of Vascular Permeability. American Journal of Physiology-Heart and Circulatory Physiology, 295, H926-H927.

https://doi.org/10.1152/ajpheart.00779.2008

[121] Cook-Mills, J.M., Marchese, M.E. and Abdala-Valencia, H. (2011) Vascular Cell Adhesion Molecule-1 Expression and Signaling During Disease: Regulation by Reactive Oxygen Species and Antioxidants. Antioxidants \& Redox Signaling, 15, 1607-1638. https://doi.org/10.1089/ars.2010.3522

[122] Meulmeester, E. and Ten Dijke, P. (2011) The Dynamic Roles of TGF- $\beta$ in Cancer. The Journal of Pathology, 223, 205-218. https://doi.org/10.1002/path.2785

[123] O’Reilly, D.A., Roberts, J.R., Cartmell, M.T., Demaine, A.G. and Kingsnorth, A.N. (2006) Heat Shock Factor-1 and Nuclear Factor-kappaB Are Systemically Activated in Human Acute Pancreatitis. JOP Journal of the Pancreas, 7, 174-184.

[124] Ling, J., Kang, Y.A., Zhao, R.Y., Xia, Q.H., Lee, D.F., Chang, Z., Li, J., Peng, B.L., Fleming, J.B., Wang, H.M., Liu, J.S., Lemischka, I.R., Hung, M.C. and Chiao, P.J. (2012) Kras ${ }^{\mathrm{G} 12 \mathrm{D}}$-Induced Ikk2/ $\beta / \mathrm{NF}-\mathrm{kB}$ Activation By IL- $1 \alpha$ and p62 Feedforward Loops Is Required for Development of Pancreatic Ductal Adenocarcinoma. Cancer Cell, 21, 105-120. https://doi.org/10.1016/j.ccr.2011.12.006 
[125] Voronov, E., Dotan, S., Krelin, Y., Song, X.P., Elkabets, M., Carmi, Y., Rider, P., Cohen, I., Romzova, M., Kaplanov, I. and Apte, R.N. (2013) Unique versus Redundant Functions of IL- $1 \alpha$ and IL- $1 \beta$ in the Tumor Microenvironment. Frontiers in Immunology, 4, 177. https://doi.org/10.3389/fimmu.2013.00177

[126] Hruban, R.H., Goggins, M., Parsons, J. and Kern, S.E. (2000) Progression Model for Pancreatic Cancer. Clinical Cancer Research, 6, 2969-2972.

[127] Matsuo, Y., Sawai, H., Ochi, N., Yasuda, A., Takahashi, H., Funahashi, H., Takeyama, H. and Guha, S. (2009) Interleukin-1 $\alpha$ Secreted by Pancreatic Cancer Cells Promotes Angiogenesis and Its Therapeutic Implications. Journal of Surgical Research, 153, 274-281. https://doi.org/10.1016/j.jss.2008.04.040

[128] Cheng, Z.X., Sun, B., Wang, S.J., Gao, Y., Zhang, Y.M., Zhou, H.X., Jia, G., Wang, Y.W., Kong, R., Pan, S.H., Xue, D.B., Jiang, H.C. and Bai, X.W. (2011) Nuclear Factor- $\mathrm{KB}-$ Dependent Epithelial to Mesenchymal Transition Induced by HIF-1 $\alpha$ Activation in Pancreatic Cancer Cells under Hypoxic Conditions. PLoS ONE, 6, e23752. https://doi.org/10.1371/journal.pone.0023752

[129] McIntyre, A., Patiar, S., Wigfield, S., Li, J.L., Ledaki, I., Turley, H., Leek, R., Snell, C., Gatter, K., Sly, W.S., Vaughan-Jones, R.D., Swietach, P. and Harris, A.L. (2012) Carbonic Anhydrase IX Promotes Tumor Growth and Necrosis in vivo and Inhibition Enhances Anti-VEGF Therapy. Clinical Cancer Research, 18, 3100-3111. https://doi.org/10.1158/1078-0432.CCR-11-1877

[130] Supuran, C.T. (2017) Carbonic Anhydrase Inhibition and the Management of Hypoxic Tumors. Metabolites, 7, 48. https://doi.org/10.3390/metabo7030048

[131] Fishel, M.L., Jiang, Y.L., Rajeshkumar, N.V., Scandura, G., Sinn, A.L., He, Y., Shen, C.Y., Jones, D.R., Pollok, K.E., Ivan, M., Maitra, A. and Kelley, M.R. (2011) Impact of APE1/Ref-1 Redox Inhibition on Pancreatic Tumor Growth. Molecular Cancer Therapeutics, 10, 1698-1708. https://doi.org/10.1158/1535-7163.MCT-11-0107

[132] Shah, F., Logsdon, D., Messmann, R.A., Fehrenbacher, J.C., Fishel, M.L. and Kelley, M.R. (2017) Exploiting the Ref-1-APE1 Node in Cancer Signaling and Other Diseases: From Bench to Clinic. npj Precision Oncology, 1, Article ID: 19. https://doi.org/10.1038/s41698-017-0023-0

[133] Logsdon, D.P., Shah, F., Carta, F., Supuran, C.T., Kamocka, M., Jacobsen, M.H., Sandusky, G.E., Kelley, M.R. and Fishel, M.K. (2018) Blocking HIF Signaling Via Novel Inhibitors of CA9 and APE1/Ref-1 Dramatically Affects Pancreatic Cancer Cell Survival. Scientific Reports, 8, Article ID: 13759.

https://doi.org/10.1038/s41598-018-32034-9

[134] Osman, M.O., Jacobsen, N.O., Kristensen, J.U., Deleuran, B., Christian, B.G., Larsen, G. and Jensen, S.L. (1998) IT 9302, a Synthetic Interleukin-10 Agonist, Diminishes Acute Lung Injury in Rabbits with Acute Necrotizing Pancreatitis. Surgery, 124, 584-592. https://doi.org/10.1016/S0039-6060(98)70106-0

[135] UniProtKB-P08151 (GLI1_HUMAN), as of 20200603.

[136] Walter, K., Omura, N., Hong, S.M., Griffith, M., Vincent, A., Borges, M. and Goggins, M. (2012) Overexpression of Smoothened Activates the Sonic Hedgehog Signaling Pathway in Pancreatic Cancer Associated Fibroblasts. Clinical Cancer Research, 16, 1781-1789. https://doi.org/10.1158/1078-0432.CCR-09-1913

[137] Lee, D.H., Lee, S.Y. and Oh, S.C. (2017) Hedgehog Signaling Pathway as a Potential Target in the Treatment of Advanced Gastric Cancer. Tumor Biology, 2017, 1-10. https://doi.org/10.1177/1010428317692266

[138] Dahmane, N., Lee, J., Robins, P., Heller, P. and Altaba, A.R.I. (1997) Activation of the Transcription Factor Gli1 and the Sonic Hedgehog Signalling Pathway in Skin 
Tumours. Nature, 389, 876-881. https://doi.org/10.1038/39918

[139] Karhadkar, S.S., Bova, G.S., Abdallah, N., Dhara, S., Gardner, D., Maitra, A., Isaacs, J.T., Berman, D.M. and Beachy P.A. (2004) Hedgehog Signalling in Prostate Regeneration, Neoplasia and Metastasis. Nature, 431, 707-712. https://doi.org/10.1038/nature02962

[140] Slade, I., Murray, A., Hanks, S., Kumar, A., Walker, L., Hargrave, D., Douglas, J., Stiller, C., Izatt, L. and Rahman, N. (2011) Heterogeneity of Familial Medulloblastoma and Contribution of Germline PTCH1 and SUFU Mutations to Sporadic Medulloblastoma. Familial Cancer, 10, Article ID: 337342. https://doi.org/10.1007/s10689-010-9411-0

[141] Berlin, J., Bendel, J.C., Hart, L.L., Firdaus, I., Gore, I., Hermann, R.C., Mulcahy, M.F., Zalupski, M.M., Mackey, H.M., Yauch, R.L., Graham, R.A., Bray, G.L. and Low, J.A. (2013) A Randomized Phase II Trial of Vismodegib versus Placebo with FOLFOX or FOLFIRI and Bevacizumab in Patients with Previously Untreated Metastatic Colorectal Cancer. Clinical Cancer Research, 19, 258-267. https://doi.org/10.1158/1078-0432.CCR-12-1800

[142] Kaye, S.B., Fehrenbacher, L., Holloway, R., Amit, A., Karlan, B., Slomovitz, B., Sabbatini, P., Fu, L., Yauch, R.L., Chang, I. and Reddy, J.C. (2012) A Phase II, Randomized, Placebo-Controlled Study of Vismodegib as Maintenance Therapy in Patients with Ovarian Cancer in Second or Third Complete Remission. Clinical Cancer Research, 18, 6509-6518. https://doi.org/10.1158/1078-0432.CCR-12-1796

[143] Morton, J.P., Mongeau, M.E., Klimstra, D.S., Morris, J.P., Lee, Y.C., Kawaguchi, Y., Wright, C.V.E., Hebro, M. and Lewis, B.C. (2007) Sonic Hedgehog Acts at Multiple Stages during Pancreatic Tumorigenesis. Proceedings of the National Academy of Sciences of the United States of America, 104, 5103-5108. https://doi.org/10.1073/pnas.0701158104

[144] Lee, K.M., Nguyen, C., Ulrich, A.B., Pour, P.M. and Ouellette, M.M. (2003) Immortalization with Telomerase of the Nestin-Positive Cells of the Human Pancreas. Biochemical and Biophysical Research Communications, 301, 1038-1044. https://doi.org/10.1016/S0006-291X(03)00086-X

[145] Infante, J.R., Matsubayashi, H., Sato, N., Tonascia, J., Klein, A.P., Riall, T.A., Yeo, C., Iacobuzio-Donahue, C. and Goggins, M. (2007) Peritumoral Fibroblast SPARC Expression and Patient Outcome with Resectable Pancreatic Adenocarcinoma. Journal of Clinical Oncology, 25, 319-325. https://doi.org/10.1200/JCO.2006.07.8824

[146] Feig, C., Gopinathan, A., Neesse, A., Chan, D.S., Cook, N. and Tuveson, D.A. (2012) The Pancreas Cancer Microenvironment. Clinical Cancer Research, 18, 4266-4276. https://doi.org/10.1158/1078-0432.CCR-11-3114

[147] Zhao, J., Wang, H., Hsiao, C.H., Chow, D.S-L., Koay, E.J., Kang, Y., Wen, X., Huang, Q., Ma, Y., Bankson, J.A., Ullrich, S.E., Overwijk, W., Maitra, A., Piwnica-Worms, D., Fleming, J.B. and Li, C. (2018) Simultaneous Inhibition of Hedgehog Signaling and Tumor Proliferation Remodels Stroma and Enhances Pancreatic Cancer Therapy. Biomaterials, 159, 215-228. https://doi.org/10.1016/j.biomaterials.2018.01.014

[148] Hidelgo, M., Amant, F., Biankin, A.V., Budinská, E., Byrne, A.T., Caldas, C., Clarke, R.B., Jong, S.D., Jonkers, J., Mælandsmo, G.M., Roman-Roman, S., Seoane, J., Trusolino, L. and Villanueva, A. (2014) Patient-Derived Xenograft Models in as an Emerging Platform for Translational Cancer Research. Cancer Discovery, 4, 998-1013. https://doi.org/10.1158/2159-8290.CD-14-0001

[149] Lee, J.W., Komar, C.A., Bengsch, F., Graham, K. and Beatty, G.L. (2016) Genetically 
Engineered Mouse Models of Pancreatic Cancer: The KPC Model (LSL-Kras ${ }^{G 12 D /+}$; $\left.L S L-T r p 53^{R 172 H /+} ; P d x-1-C r e\right)$, Its Variants and Their Application in Immuno-Oncology Drug Discovery. Current Protocols in Pharmacology, 73, 14.39.114.39.20. https://doi.org/10.1002/cpph.2

[150] Rosler, A., Vandermeulen, G.W. and Klok, H.A. (2001) Advanced Drug Delivery Devices via Self-Assembly of Amphiphilic Block Copolymers. Advanced Drug Delivery Reviews, 53, 95-108. https://doi.org/10.1016/S0169-409X(01)00222-8

[151] Aster, J.C. (2014) In Brief: Notch Signalling in Health and Disease. The Journal of Pathology, 232, 1-3. https://doi.org/10.1002/path.4291

[152] Li, X.Y., Zhai, W.J. and Teng, C.B. (2016) Notch Signaling in Pancreatic Development. International Journal of Molecular Sciences, 17, 48. https://doi.org/10.3390/ijms17010048

[153] Bray, S.J. (2006) Notch Signalling: A Simple Pathway Becomes Complex. Nature Reviews Molecular Cell Biology, 7, 678-689. https://doi.org/10.1038/nrm2009

[154] Dikic, I. and Schimidt, M.H. (2010) Notch: Implications of Endogenous Inhibitors for Therapy. BioEssays, 32, 481-487. https://doi.org/10.1002/bies.200900140

[155] Akanuma, T., Chen, C., Sato, T., Merks, R.M. and Sato T.N. (2016) Memory of Cell Shape Biases Stochastic Fate Decision-Making despite Mitotic Rounding. Nature Communications, 7, Article ID: 11963. https://doi.org/10.1038/ncomms11963

[156] Kopan, R. and Ilagan, M.X. (2009) The Canonical Notch Signalling Pathway: Unfolding the Activation Mechanism. Cell, 137, 216-233.

https://doi.org/10.1016/j.cell.2009.03.045

[157] Gao, J., Long, B. and Wang, Z. (2017) Role of Notch Signaling Pathway in Pancreatic Cancer. American Journal of Cancer Research, 7, 173-186.

[158] Kageyama, R., Ohtsuka, T. and Kobayashi, T. (2007) The Hes Gene Family: Repressors and Oscillators that Orchestrate Embryogenesis. Development, 134, 1243-1251. https://doi.org/10.1242/dev.000786

[159] HEY1 Hes Related Family bHLH Transcription Factor with YRPW Motif 1 [Homo sapiens (Human)], NCBI, Full Report, Gene ID: 23462, Updated on 24-Mar-2020.

[160] Chillakuri, C.R., Sheppard, D., Lea, S.M. and Handford, P.A. (2012) Notch Receptor-Ligand Binding and Activation: Insights from Molecular Studies. Seminars in Cell \& Developmental Biology, 23, 421-428. https://doi.org/10.1016/j.semcdb.2012.01.009

[161] Joussineau, C.D., Soulé, J., Martin, M., Anguille, C., Montcourrier, P. and Alexandre, D. (2003) Delta-Promoted Filopodia Mediate Long-Range Lateral Inhibition in Drosophila. Nature, 426, 555-559. https://doi.org/10.1038/nature02157

[162] Abel, E.V., Kim, E.J., Wu, J., Hynes, M., Bednar, F., Proctor, E., Wang, L., Dziubinski, M.L. and Simeone, D.M. (2014) The Notch Pathway Is Important in Maintaining the Cancer Stem Cell Population in Pancreatic Cancer. PLoS One, 9, e91983. https://doi.org/10.1371/journal.pone.0091983

[163] Li, C., Heidt, D.G., Dalerba, P., Burant, C.F., Zhang, L., Adsay, V., Wicha, M., Clarke, M.F. and Simeone, D.M. (2007) Identification of Pancreatic Cancer Stem Cells. Cancer Research, 67, 1030-1037. https://doi.org/10.1158/0008-5472.CAN-06-2030

[164] Richter, S., Mcwhirter, E., Chen, E.X. and Tran, B. (2012) A Phase I Study of R04929097, an Oral Gamma Secretase Inhibitor, in Combination with Gemcitabine in Patients with Advanced Solid Tumors (PHL-078/CTEP 8575). Journal of Clinical 
Oncology, 30, 3082-3082. https://doi.org/10.1200/jco.2012.30.15 suppl.3082

[165] Jesus-Acosta, A., Laheru, D., Maitra, A., Arcaroli, J., Rudek, M.A., Dasari, A., Blatchford, P.J., Quackenbush, K. and Messersmith, W. (2014) A Phase II Study of the Gamma Secretase Inhibitor R04929097 in Patients Previously Treated Metastatic Pancreatic Adenocarcinoma. Investigational New Drugs, 32, 739-745. https://doi.org/10.1007/s10637-014-0083-8

[166] Fung, P.C.W. and Kong, R.K.C. (2016) The Integrative Five-Fluid Circulation System in the Human Body. Open Journal of Molecular and Integrative Physiology, 6, 45-97. https://doi.org/10.4236/ojmip.2016.64005

[167] Zha, M., Li, F., Xu, W., Chen, B. and Sun, Z. (2014) Isolation and Characterization of Islet Stellate Cells in Rat. Islets, 4, Article ID: e28701. https://doi.org/10.4161/isl.28701

[168] Apte, M.V., Pirola, R.C. and Wilson, J.S. (211) Pancreatic Stellate Cells: A Starring Role in Normal and Diseased Pancreas. Frontiers in Physiology, 3, 344.

https://doi.org/10.3389/fphys.2012.00344

[169] McCarroll, J.A., Phillips, P.A., Santucci, N., Pirola, R.C., Wilson, J.S. and Apte, M.V. (2006) Vitamin A Inhibits Pancreatic Stellate Cell Activation: Implication for Treatment of Pancreatic Fibrosis. Gut, 55, 79-89.

https://doi.org/10.1136/gut.2005.064543

[170] Erkan, M., Kleeff, J., Gorbachevski, A., Reiser, C., Mitkus, T., Esposito, I., Giese, T., Büchler, M.W., Giese, N.A. and Friess, H. (2007) Periostin Creates a Tumor-Supportive Microenvironment in the Pancreas by Sustaining Fibrogenic Stellate Cell Activity. Gastroenterology, 132, 1447-1464.

https://doi.org/10.1053/j.gastro.2007.01.031

[171] Zilberberg, L., Vesna Todorovic, V., Dabovic, B., Horiguchi, M., Couroussé, T., Sakai, 1.Y. and Rifkin, D.B. (2012) Specificity of Latent TGF-ß Binding Protein (LTBP) Incorporation into Matrix: Role of Fibrillins and Fibronectin. Journal of Cellular Physiology, 227, 3828-3836. https://doi.org/10.1002/jcp.24094

[172] Fung, P.C.W. (2009) Probing the Mystery of Chinese Medicine Meridian Channels with Special Emphasis on the Connective Tissue Interstitial Fluid System, Mechanotransduction, Cells Durotaxis and Mast Cell Degranulation. Chinese Medicine, 4, 10. https://doi.org/10.1186/1749-8546-4-10

[173] Munger, J.S. and Sheppard, D. (2011) Cross Talk among TGF-Beta Signal Pathways, Integrins and the ECM. Cold Spring Harbor Perspectives in Biology, 3, Article ID: a005017. https://doi.org/10.1101/cshperspect.a005017

[174] Han, X., Xu, Y., Zhao, X., Zhang, Y., Yang, X., Wang, Y., Zhao, R., Anderson, G.J., Zhao, Y. and Nie, G. (2018) Reversal of Pancreatic Desmoplasia by Re-Educating Stellate Cells with a Tumour Microenvironment-Activated Nanosystem. Nature Communications, 9, Article ID: 3390. https://doi.org/10.1038/s41467-018-05906-x

[175] Ren, B., Cui, M., Yang, G., Wang, H., Feng, M., You, L. and Zhao, Y. (2018) Tumor Microenvironment Participates in Metastasis of Pancreatic Cancer. Molecular Cancer, 17, Article 108, 1-15. https://doi.org/10.1186/s12943-018-0858-1

[176] Haber, P.S., Keogh, G.W., Apte, M.V., Moran, C.S., Stewart, N.L., Crawford, D.H., Pirola, R.C., McCaughan, G.W., Ramm, G.A. and Wilson, J.S. (1999) Activation of Pancreatic Stellate Cells in Human and Experimental Pancreatic Fibrosis. The American Journal of Pathology, 155, 1087-1095. https://doi.org/10.1016/S0002-9440(10)65211-X

[177] Jun, J.I. and Lau, L.F. (2011) Taking Aim at the Extracellular Matrix: CCN Proteins 
as Emerging Therapeutic Targets. Nature Reviews Drug Discovery, 10, 945-963. https://doi.org/10.1038/nrd3599

[178] Charrier, A.L. and Brigstock, D.R. (2010) Connective Tissue Growth Factor Production by Activated Pancreatic Stellate Cells in Mouse Alcoholic Chronic Pancreatitis. Laboratory Investigation, 90, 1179-1188.

https://doi.org/10.1038/labinvest.2010.82

[179] Jin, G., Hong, W., Guo, Y., Bai, Y. and Chen, B. (2020) Molecular Mechanism of Pancreatic Cell Activation in Chronic Pancreatitis and Pancreatic Cancer. Journal of Cancer, 11, 1505-1515. https://doi.org/10.7150/jca.38616

[180] Luttenberger, T., Schmid-Kotsas, A., Menke, A., Siech, M., Beger, H., Adler, G., Grünert, A. and Bachem, M.G. (2000) Platlet-Derived Growth Factors Stimulate Prolin and Extracellular Matrix Synthesis of Pancreatic Stellate Cells: Implication in Pathogenesis of Pancreas Fibrous. Laboratory Investigation, 80, 47-55. https://doi.org/10.1038/labinvest.3780007

[181] Masamune, A. (2013) Connexins Regulate Cell Functions in Pancreatic Stellate Cells. Pancreas, 42, 308-316. https://doi.org/10.1097/MPA.0b013e31825c51d6

[182] Delmos, A., Van Laethem, J.L., Quertinmont, E., Degraef, C., Delhaye, M., Geerts, A. and Deviere, J. (2002) Endogenous Interleukin-10 Modulates Fibrosis and Regeneration in Experimental Chronic Pancreatitis. American Journal of Physiology-Gastrointestinal and Liver Physiology, 282, G1105-G1112. https://doi.org/10.1152/ajpgi.00431.2001

[183] Driessler, F., Venstrom, K., Sabat, R., Asadullah, K. and Schottelius, A.J. (2004) Molecular Mechanisms of Interleukin-10-Mediated Inhibition of NF-kB Activity: A Role for p50 F. Clinical \& Experimental Immunology, 135, 64-73. https://doi.org/10.1111/j.1365-2249.2004.02342.x

[184] Carey, A.J., Tan, C.K. and Ulett, G.C. (2012) Infection-Induced IL-10 and JAK-STAT a Review of the Molecular Circuitry Controlling Immune Hyperactivity in Response to Pathogenic Microbes. JAK-STAT, 3, 159-167.

https://doi.org/10.4161/jkst.19918

[185] Asaumi, H., Watanabe, S., Taguchi, M., Tashiro, M. and Otsuki, M. (2007) Externally Applied Pressure Activates Pancreatic Stellate Cells through the Generation of Intracellular Reactive Oxygen Species. American Journal of Physiology-Gastrointestinal and Liver Physiology, 293, G972-G978. https://doi.org/10.1152/ajpgi.00018.2007

[186] McGavran, M.H., Unger, R.H., Recant, L., Polk, H.C., Kilo, C. and Levin, M.E. (1966) A Glucagon-Secreting Alpha-Cell Carcinoma of the Pancreas. New England Journal of Medicine, 274, 1408-1413. https://doi.org/10.1056/NEJM196606232742503

[187] Kim, S.K., Kim, H., Lee, D.-H., Kim, T.-S., Kim, T., Chung, C., Koh, G.Y., Kim, H. and Lim. D.S. (2013) Reversing the Intractable Nature of Pancreatic Cancer by Selectively Targeting ALDH-High, Therapy-Resistant Cancer Cells. PLoS ONE, 8, e78130. https://doi.org/10.1371/journal.pone.0078130

[188] Ginestier, C., Hur, M.H., Charafe-Jauffret, E., Monville, F., Dutcher, J., Brown, M., Jacquemier, J., Viens, P., Kleer, C.G., Liu, S., Schott, A., Hayes, D., Birnbaum, D., Wicha, M.S. and Dontu, G. (2007) ALDH1 Is a Marker of Normal and Malignant Human Mammary Stem Cells and a Predictor of Poor Clinical Outcome. Cell, Stem Cell, 1, 555-567. https://doi.org/10.1016/j.stem.2007.08.014

[189] CD24 CD24 Molecule [Homo sapiens (Human)], Gene ID: 100133941, NCBI Full Report Updated on 20-Sep-2020.

[190] Wei, H.J., Yin, T., Zhu, Z., Shi, P.F., Tian, Y. and Wang, C.Y. (2011) Expression of 
CD44, CD24 and ESA in Pancreatic Adenocarcinoma Cell Lines Varies with Local Microenvironment. Hepatobiliary \& Pancreatic Diseases International, 10, 428-434. https://doi.org/10.1016/S1499-3872(11)60073-8

[191] Lin, L., Jou, D., Wang, Y., Ma, H., Lin, T., Fuchs, J., Li, P.K., Lu, J., Li, C. and Lin, J. (2016) Stats3 as a Potential Therapeutic Target in ALDH+ and CD44+/CD24+ Stem Cell-Like Pancreatic Cancer Cells. International Journal of Oncology, 49, 2265-2274. https://doi.org/10.3892/ijo.2016.3728

[192] Perez, A., Neskey, D.M., Wen, J., Pereira, L., Reategui, E.P., Goodwin, W.J., Carraway, K.L. and Franzmann, E.J. (2013) CD44 Interacts with EGFR and Promotes Head and Neck Squamous Cell Carcinoma Initiation and Progression. Oral Oncology, 49, 306-313. https://doi.org/10.1016/j.oraloncology.2012.11.009

[193] Yin, J., Zhang, H., Wu, X., Zhang, Y., Li, J., Shen, J., Zhao, Y., Xiao, Z., Lu, L., Huang, C., Zhang, Z., Du, F., Wu, Y., Kaboli, P.J., Cho, C.H., Yuan, D. and Li, M. (2020) CD44 Inhibition Attenuates EGFR Signaling and Enhances Cisplatin Sensitivity in Human EGFR Wild-Type Non-Small-Cell Lung Cancer Cells. International Journal of Molecular Medicine, 45, 1783-1792. https://doi.org/10.3892/ijmm.2020.4562

[194] Pantel, K., Brakenhoff, R.H. and Brandt, B. (2008) Detection, Clinical Relevance and Specific Biological Properties of Disseminating Tumour Cells. Nature Reviews Cancer, 8, 329-340. https://doi.org/10.1038/nrc2375

[195] Greco, F.A. and Hainsworth, J.D. (2009) Introduction: Unknown Primary Cancer. Seminars in Oncology, 36, 6-7. https://doi.org/10.1053/j.seminoncol.2008.10.007

[196] Gupta, P.B., Mani, S., Yang, J., Hartwell, K. and Weinberg, R.A. (2005) The Evolving Portrait of Cancer Metastasis. Cold Spring Harbor Perspectives in Biology, 70, 291-297. https://doi.org/10.1101/sqb.2005.70.033

[197] Han, S.P. and Yap, A.S. (2012) The Cytoskeleton and Classical Adhesions. Subcellular Biochemistry, 60, 111-135. https://doi.org/10.1007/978-94-007-4186-7 6

[198] Brieher, W.M. and Yap, A.S. (2012) Cadherin Junctions and Their Cytoskeleton(s). Current Opinion in Cell Biology, 25, 39-46.

https://doi.org/10.1016/j.ceb.2012.10.010

[199] Ribeiro, A.S. and Paredes, J. (2015) P-Cadherin Linking Breast Can Invasion: A Promising Marker to Identify an "Intermediate/Metastable" EMT State. Frontiers in Oncology, 4, Article ID: 371. https://doi.org/10.3389/fonc.2014.00371

[200] Storz, P. (2017) Acinar Cell Plasticity and Development of Pancreatic Ductal Adenocarcinoma. Nature Reviews Gastroenterology \& Hepatology, 14, 296-304.

https://doi.org/10.1038/nrgastro.2017.12

[201] Rhim, A.D., Thege, F.I., Santana, S.M., Lannin, T.B., Saha, T.N., Tsai, S., Maggs, L.R., Kochman, M.L., Ginsberg, G.G., Lieb, J.G., Chandrasekhara, V., Drebin, J.A., Ahmad, N., Yang, Y.X., Kirby, B.J. and Stanger, B.Z. (2014) Detection of Circulating Pancreas Epithelial Cells in Patients with Pancreatic Cystic Lesions. Gastroenterology, 146, 647-651. https://doi.org/10.1053/j.gastro.2013.12.007

[202] Pećina-Šlaus, N. (2003) Tumor Suppressor Gene E-Cadherin and Its Role in Normal and Malignant Cells. Cancer Cell International, 3, Article 17, 1-7. https://doi.org/10.1186/1475-2867-3-17

[203] Gu, G., Brown, J.R. and Melton, D.A. (2003) Direct Lineage Tracing Reveals the Ontogeny of Pancreatic Cell Fates during Mouse Embryogenesis. Mechanisms of Development, 120, 35-43. https://doi.org/10.1016/S0925-4773(02)00330-1

[204] Rhim, A.D., Mirek, E.T., Aiello, N.M., Maitra, A., Bailey, J.M., McCallister, F., Reichert, M., Beatty, G.L., Rustgi, A.K., Vonderheide, R.H., Leach, S.D. and Stanger, 
B.Z. (2012) EMT and Dissemination Precede Pancreatic Tumor Formation. Cell, 148, 349-361. https://doi.org/10.1016/j.cell.2011.11.025

[205] Masugi, Y., Yamazak, I.K., Hibi, T., Aiura, K., Kitagawa, Y. and Sakamoto, M. (2010) Solitary Cell Infiltration Is a Novel Indicator of Poor Prognosis and Epithelial-Mesenchymal Transition in Pancreatic Cancer. Human Pathology, 41, 1061-1068. https://doi.org/10.1016/j.humpath.2010.01.016

[206] Aruffo, A., Stamenkovic, I., Melnick, M., Underhill, C.B. and Seed, B. (1990) CD44 Is the Principal Cell Surface Receptor for Hyaluronate. Cell, 61, 1303-1313. https://doi.org/10.1016/0092-8674(90)90694-A

[207] Jiang, W., Zhang, Y., Kane, K.T., Collins, M.A., Simeone, D.M., di Magliano, M.P. and Nguyen, K.T. (2015) CD44 Regulates Pancreatic Cancer Invasion through MT1-MMP. Molecular Cancer Research, 13, 9-15. https://doi.org/10.1158/1541-7786.MCR-14-0076

[208] Chang, T.T., Thakar, D. and Weaver, V.M. (2017) Force-Dependent Breaching of the Basement Membrane. Matrix Biology, 57/58, 178-189. https://doi.org/10.1016/j.matbio.2016.12.005

[209] O’Leary, L.E.R., Fallas, J.A., Bakota, E.L, Kang, M.K. and Hartgerink, J.D. (2011) Multi-Hierarchical Self-Assembly of a Collagen Mimetic Peptide from Triple Helix to Nanofibre and Hydrogel. Nature Chemistry, 3, 821-828. https://doi.org/10.1038/nchem.1123

[210] Monteiro, P., Rosse, C., Castro-Castro, A., Irondellel, M., Lagoutte, E., Paul-Gillotreaux, P., Desnos, C., Formstcher, E., Darchen, F., Perrais, D., Gautreau, A., Hertzog, M. and Chavrier, P. (2013) Endosomal WASH and Exocyst Complexes Control Exocytosis of MT1-MMP at Uinvadopodia. Journal of Cell Biology, 203, 1063-1079. https://doi.org/10.1083/jcb.201306162

[211] Marchesin, V., Castro-Castro, A., Lodillinsky, C., Castagnino, A., Cyrta, J., Bonsang-Kitzis, H., Fuhrmann, L., Irondelle, M., Infante, E., Montagnac, G., Reyal, F., Vincent-Salomon, A. and Chevrier, P. (2015) ARF6-JIP3/4 Regulate Endosomal Tubules for MT1-MMP Exocytosis in Cancer Invasion. Journal of Cell Biology, 211, 339-358. https://doi.org/10.1083/jcb.201506002

[212] Castro-Castro, A., Marchesin, V., Monteiro, P., Lodillinsky, C., Rosse, C. and Chavrier, P. (2016) Cellular and Molecular Mechanisms of MT1-MMP-Dependent Cancer Cell Invasion. Annual Review of Cell and Developmental Biology, 32, 555-576. https://doi.org/10.1146/annurev-cellbio-111315-125227

[213] Hoyer, M.J., Chitwood, P.J., Ebmeier, C.C., Striepen, J.F., Qi, R.Z., Old, W.M. and Voeltz, G.K. (2018) A Novel Class of ER Membrane Proteins Regulates ER-Associated Endosome Fission. Cell, 175, 254-265. https://doi.org/10.1016/j.cell.2018.08.030

[214] Pedersen, N.M., Wenzel, E.M., Wang, L., Antoine, S., Chavrier, P., Stenmark, H. and Raiborg, C. (2020) Protrudin-Mediated ER-Endosome Contact Sites Promote MT1-MMP Exocytosis and Cell Invasion. Journal of Cell Biology, 219, e2020 03063. https://doi.org/10.1083/jcb.202003063

[215] Fisher, K.E., Sacharidou, A., Stratman, A.N., Mayo, A.M., Fisher, S.B., Mahan, R.D., Davis, M.J. and Davis, G.E. (2009) MT1-MMP- and Cdc42-Dependent Signaling Co-Regulate Cell Invasion and Tunnel Formation in 3D. Collagen Matrices. Journal of Cell Science, 122, 4558-4569. https://doi.org/10.1242/jcs.050724

[216] Ortenos, C., Ehlert, R. and Gollnick, H. (1987) The Retinoids: A Review of Their Clinical Pharmacologic and Therapeutic Use. Drugs, 34, 459-503. https://doi.org/10.2165/00003495-198734040-00003

[217] Bold, R.J., Ishizuka, J., Townsend Jr., C.M. and Thompson, J.C. (1996) 
All-Trans-Retinoic Acid Inhibits Growth of Human Pancreatic Cancer Cell Lines. Pancreas, 12, 189-195. https://doi.org/10.1097/00006676-199603000-00014

[218] Chronopoulos, A., Robinson, B., Sarper, M., Cortes, E., Hurnheimar, V., Lachoqwski, D., Attwood, S., Garcia, R., Ghassemi, S., del Rio Fabry, B. and Hernandez, A. (2016) ATRA Mechanically Reprograms Pancreatic Stellar Cells to Supress Matrix Remodeling and Inhibition of Cancer Cell Invasion. Nature Communications, I7, Article ID: 126301I. https://doi.org/10.1038/ncomms12630

[219] Fairus, S., Nor, R.M., Cheng, H.M. and Sundram, K. (2006) Postprandial Metabolic Fate of Tocotrienol-Rich Vitamin E Differs Significantly from that of Alpha-Tocopherol. The American Journal of Clinical Nutrition, 84, 835-842. https://doi.org/10.1093/ajcn/84.4.835

[220] Fu, J.Y., Che, H.L., Tan, D.M.Y. and Teng, K.T. (2014) Bioavailability of Tocotrienols: Evidence in Human Studies. Nutrition \& Metabolism, 11, 5. https://doi.org/10.1186/1743-7075-11-5

[221] Fu, J.Y., Blatchford, D.R., Tetley, L. and Dufes, C. (2009) Tumor Regression after Systemic Administration of Tocotrienol Entrapped in Tumor-Targeted Vesicles. Journal of Controlled Release, 140, 95-99. https://doi.org/10.1016/j.jconrel.2009.08.017

[222] Miyosh, N., Wakao, Y., Tomono, S., Tatemichi, M., Yano, T. and Ohshima, H. (2011) The Enhancement of the Oral Bioavailability of Gamma-Tocotrienol in Mice by Gamma-Cyclodextrin Inclusion. The Journal of Nutritional Biochemistry, 22, 1121-1126. https://doi.org/10.1016/j.jnutbio.2010.09.011

[223] Fu, J.Y., Zhang, W., Blatchford, D.R., Tetley, L., McConnell, G. and Dufes, C. (2011) Novel Tocotrienol-Entrapping Vesicles Can Eradicate Solid Tumors. Journal of Controlled Release, 154, 20-26. https://doi.org/10.1016/j.jconrel.2011.04.015

[224] Husain, K., Francois, R.A., Yamauchi, T., Pereg, M., Sebti, S.M. and Malafa, M.P. (2011) Vitamin E Delta-Tocotrienol Augments the Antitumor Activity of Gemcitabine and Suppresses Constitutive NF-k B Action in Pancreatic Cancer. Molecular Cancer Therapeutics, 10, 2363-2372. https://doi.org/10.1158/1535-7163.MCT-11-0424

[225] Husain, K., Centeno, B.A., Coppla, D., Trevisso, J., Sebti, S.M. and Malafa, M.P. (2017) Delta-Tocotrienol, a Natural Form of Vitamin E, Inhibits Pancreatic Cancer Stem-Like Cells and Prevents Pancreatic Cancer Metastasis. Oncotarget, 8, 31554-31567. https://doi.org/10.18632/oncotarget.15767

[226] Vitamin D Receptor-Mediated Stromal Reprogramming Supresses Pancreatitis and Enhances Pancreatic Cancer Therapy. Cell, 159, 80-93.

[227] Hingorani, S.R., Wang, L., Multani, A.S., Combs, C., Therese B Deramaudt, T.B., Hruban, R.H., Rustgi, A.K., Chang, S. and Tuveson, D.A. (2005) Trp53R172H and KrasG12D Cooperate to Promote Chromosomal Instability and Widely Metastatic Pancreatic Adenocarcinoma. Cancer Cell, 7, 469-483. https://doi.org/10.1016/j.ccr.2005.04.023

[228] Kong, F., Li, L., Wang, G., Deng, X., Li, Z. and Kong, X. (2019) VDR Signaling Inhibits Cancer Associated-Fibroblasts' Release of Exosomal miR-10a-5p and Limits Their Supportive Effects on Pancreatic Cancer Cells. Gut, 68, 950-951. https://doi.org/10.1136/gutjnl-2018-316627

[229] Sato, N., Kohi, S., Hirata, K. and Goggins, M. (2016) Role of Hyaluronan in Pancreatic Cancer Biology and Therapy: Once again in the Spotlight. Cancer Science, 107, 567-575. https://doi.org/10.1111/cas.12913 
[230] Shepard, H.M. (2015) Breaching the Castle Walls: Hyaluronan Depletion as a Therapeutic Approach to Cancer Therapy. Frontiers in Oncology, 5, 192. https://doi.org/10.3389/fonc.2015.00192

[231] Thompson, C.B., Shepard, H.M., Kadhim, S., Jiang, P. and Osgood, R.J. (2010) Enzymatic Depletion of Tumor Hyaluronan Induces Antitumor Responses in Preclinical Animal Models. Molecular Cancer Therapeutics, 9, 3052-3064. https://doi.org/10.1158/1535-7163.MCT-10-0470

[232] O’Brien, J., Hayder, H., Zayed, Y. and Peng, C. (2018) Overview of MicroRNA Biogenesis, Mechanisms of Actions, and Circulation. Frontiers in Endocrinology, 9, 402. https://doi.org/10.3389/fendo.2018.00402

[233] Zhang, H. and Lu, W. (2018) LncRNA SNHG12 Regulates Gastric Cancer Progression by Acting as a Molecular Sponge of miR-320. Molecular Medicine Reports, 17, 2743-2749. https://doi.org/10.3892/mmr.2017.8143

[234] Yeung, T.L., Leung, C.S., Wong, K.K., Gutierrez-Hartmann, A., Kwong, J., Gershenson, D.M. and Mok, S.C. (2017) ELF3 Is a Negative Regulator of Epithelial-Mesenchymal Transition in Ovarian Cancer Cells. Oncotarget, 8, 16951-16963. https://doi.org/10.18632/oncotarget.15208

[235] Zhang, Z.Q., Zhang, J.K., Li, J.M., Geng, H.J., Zhou, B.J., Zhang, B.X. and Chen, H. (2020) miR-320/ELF3 Axis Inhibits the Progression of Breast Cancer via the PI3K/AKT Pathway. Oncology Letters, 19, 3239-3248.

https://doi.org/10.3892/ol.2020.11440

[236] Almoguera, C., Shibata, D., Forrester, K., Martin, J., Arnheim, N. and Perucho, M. (1988) Most Human Carcinomas of the Exocrine Pancreas Contain Mutant c-K-ras Genes. Cell, 53, 549-554. https://doi.org/10.1016/0092-8674(88)90571-5

[237] Campbell, P.M., Groehler, A.L., Lee, K.M., Ouellette, M.M., Khazak, V. and Der, C.J. (2007) K-Ras Promotes Growth Transformation and Invasion of Immortalized Human Pancreatic Cells by Raf and Phosphatidylinositol 3-Kinase Signaling. Cancer Research, 67, 2098-2106. https://doi.org/10.1158/0008-5472.CAN-06-3752

[238] Kallifatidis, G., Rausch, V., Baumann, B., Apel, A., Beckermann, B.M., Groth, A., Mattern, J., Li, Z., Kolb, A., Moldenhauer, G., Altevogt, P., Wirth, T., Werner, J., Schemmer, P., Büchler, M.W., Salnikov, A.V. and Herr, I. (2009) Sulforaphane Targets Pancreatic Tumour-Initiating Cells by NF-kB-Induced Antiapoptotic Signalling. Gut, 58, 949-963. https://doi.org/10.1136/gut.2008.149039

[239] Agbunag, C. and Bar-Sagi, D. (2004) Oncogenic K-Ras Drives Cell Cycle Progression and Phenotypic Conversion of Primary Pancreatic Duct Epithelial Cells. Cancer Research, 64, 5659-5663.

https://doi.org/10.1158/0008-5472.CAN-04-0807

[240] Yu, S.N., Lu, Z.H., Liu, C.Z., Meng, Y.X., Ma, Y.H., Zhao, W.G., Liu, J.P., Yu, J. and Chen, J. (2010) miRNA-96 Suppresses KRAS and Functions as a Tumor Suppressor Gene in Pancreatic Cancer. Cancer Research, 70, 6015-6025. https://doi.org/10.1158/0008-5472.CAN-09-4531

[241] Apelqvist, Å., Li, H., Sommer, L., Beatus, P., Anderson, D.J., Honjo, T., de Angelis, M.H., Lendahl, U. and Edlund, H. (1999) Notch Signalling Controls Pancreatic Cell Differentiation. Nature, 400, 877-881. https://doi.org/10.1038/23716

[242] Raver-Shapira, N., Marciano, E., Meiri, E., Spector, Y., Rosenfeld, N., Moskovits, N., Bentwich, Z. and Oren, M. (2007) Transcriptional Activation of miR-34a Contributes to p53-Mediated Apoptosis. Molecular Cell, 26, 731-743. https://doi.org/10.1016/j.molcel.2007.05.017

[243] Perumalsamy, L.R., Nagala, M. and Sarin, A. (2010) Notch-Activated Signaling 
Cascade Interacts with Mitochondrial Remodeling Proteins to Regulate Cell Survival. PNAS, 107, 6882-6887. https://doi.org/10.1073/pnas.0910060107

[244] Ji, Q., Hao, X., Zhang, M., Tang, W., Yang, M., Li, L., Xiang, D., Desano, J.T., Bommer, G.T., Fan, D., Fearon, E.R., Lawrence, T.S. and Xu, L. (2009) MicroRNA miR-34 Inhibits Human Pancreatic Cancer Tumor-Initiating Cells. PLoS ONE, 4, e6816. https://doi.org/10.1371/journal.pone.0006816

[245] Niess, H., Camaj, P., Renner, A., Ischenko, I., Zhao, Y., Krebs, S., Mysliwietz, J., Jäckel, C., Nelson, P.J., Blum, H., Jauch, K.W., Ellwart, J.W. and Bruns, C.J. (2014) Side Population Cells of Pancreatic Cancer Show Characteristics of Cancer Stem Cells Responsible for Resistance and Metastasis. Targeted Oncology, 10, 215-227. https://doi.org/10.1007/s11523-014-0323-Z

[246] Zhao, Y., Zhao, L., Ischenko, I., Bao, Q., Schwarz, B., Nieß, H., Wang, Y., Renner, A., Mysliwietz, J., Jauch, K.W., Nelson, P.J., Ellwart, J.W., Bruns, C.J. and Camaj, P. (2015) Antisense Inhibition of microRNA-21 and microRNA-221 in Tumor-Initiating Stem-Like Cells Modulates Tumorigenesis, Metastatic, and Chemotherapy Resistance in Pancreatic Cancer. Targeted Oncology, 10, 535-548. https://doi.org/10.1007/s11523-015-0360-2

[247] Park, J.K., Lee, E.J., Esau, C. and Schmittgen, T.D. (2009) Antisense Inhibition of miroRNA-21 or -221 Arrests Cell Cycle, Induces Apoptosis, and Sensitizes the Effects of Gemcitabine in Pancreatic Adenocarcinoma. Pancreas, 38, e190-e199. https://doi.org/10.1097/MPA.0b013e3181ba82e1

[248] Li, Y., Vanderboom, T.G., Wang, Z., Kong, D., Ali, S., Philip, P.A. and Sarkar, F.H. (2010) miR-146a Suppresses Invasion of Pancreatic Cancer Cells. Cancer Research, 70, 1486-1495. https://doi.org/10.1158/0008-5472.CAN-09-2792

[249] Si, W., Liu, X., Wei, R., Zhang, Y., Zhao, Y., Cui, L. and Hong, T. (2019) MTA2-Mediated Inhibition of PTEN Leads to Pancreatic Ductal Adenocarcinoma Carcinogenesis. Cell Death \& Disease, 10, 206.

https://doi.org/10.1038/s41419-019-1424-5

[250] Bahn, J.H., Zhang, Q., Li, F., Chan, T.M., Lin, X.Z., Kim, Y., Wong, D.T.W. and Xiao, X.S. (2015) The Landscape of MicroRNA, Piwi-Interacting RNA, and Circular RNA in Human Saliva. Clinical Chemistry, 61, 221-230. https://doi.org/10.1373/clinchem.2014.230433

[251] Liu, J., Zhang, X., Yan, M.N. and Li, H. (2020) Emerging Role of Circular RNAs in Cancer. Frontiers in Oncology, 10, 663. https://doi.org/10.3389/fonc.2020.00663

[252] Ma, L.N., Liu, J., Shen, J.J., Liu, L., Wu, J., Li, W., Luo, J.J., Chen, Q. and Qian, C. (2010) Expression of miR-122 Mediated by Adenoviral Vector Induces Apoptosis and Cell Cycle Arrest of Cancer Cells. Cancer Biology \& Therapy, 9, 554-561. https://doi.org/10.4161/cbt.9.7.11267

[253] Gui, Y., Liu, H., Zhang, L., Lv, W. and Hu, X. (2015) Altered microRNA Profiles in Cerebrospinal Fluid Exosome in Parkinson Disease and Alzheimer Disease. Oncotarget, 6, 37043-37053. https://doi.org/10.18632/oncotarget.6158

[254] Kölling, M., Haddad, G., Wegmann, U., Kistler, A., Bosakova, A., Seeger, H., Hübel, K., Haller, H., Mueller, T., Wüthrich, R.P. and Lorenzen, J.M. (2019) Transplant Patients with Acute T Cell-Mediated Allograft Rejection. Clinical Chemistry, 65, 1287-1294. https://doi.org/10.1373/clinchem.2019.305854

[255] Li, H.M., Hao, X.K., Wang, H.M., Liu, Z.C., He, Y., Pu, M., Zhang, H.T., Yu, H.C., Duan, J.L. and Qu, S.B. (2016) Circular RNA Expression Profile of Pancreatic Ductal Adenocarcinoma Revealed by Microarray. Cell Physiol Biochem, 40, 1334-1344. https://doi.org/10.1159/000453186 
[256] Li, J., Li, Z.H., Jiang, P., Peng, M.J., Zhang, X., Chen, K., Liu, H., Bi, H.Q., Liu, X.D. and Li, X.W. (2018) Circular RNA IARS (circ-IARS) Secreted by Pancreatic Cancer Cells and Located Within Exosomes Regulates Endothelial Monolayer Permeability to Promote Tumor Metastasis. Journal of Experimental \& Clinical Cancer Research, 37, Article ID: 177. https://doi.org/10.1186/s13046-018-0822-3

[257] UniProtKB-Q07157 (ZO1_HUMAN).

[258] Yang, F., Liu, D.Y., Guo, J.T., Ge, N., Zhu, P., Liu, X., Wang, S., Wang, G.X. and Sun, S.Y. (2017) Circular RNA circ-LDLRAD3 as a Biomarker in Diagnosis of Pancreatic Cancer. World Journal of Gastroenterology, 23, 8345-8354. https://doi.org/10.3748/wjg.v23.i47.8345

[259] Chen, G., Shi, Y., Zhang, Y. and Sun, J. (2017) CircRNA_100782 Regulates Pancreatic Carcinoma Proliferation through the IL6-STAT3 Pathway. OncoTargets and Therapy, 10, 5783-5794. https://doi.org/10.2147/OTT.S150678

[260] Hao, L.G., Rong, W., Bai, L.J., Cui, H.S., Zhang, S.L., Li, Y.C., Chen, D.T. and Meng, X. (2018) Upregulated Circular RNA circ_0007534 Indicates an Unfavorable Prognosis in Pancreatic Ductal Adenocarcinoma and Regulates Cell Proliferation, Apoptosis, and Invasion by Sponging miR-625 and miR-892b. Journal of Cellular Biochemistry, 120, 3780-3789. https://doi.org/10.1002/jcb.27658

[261] Li, X.L., Wu, Z.Q., Fu, X.B. and Han, W.D. (2014) LncRNA: Insights into Their Function and Mechanics in Underlying Disorders. Mutation Research-Reviews in Mutation Research, 762, 1-21. https://doi.org/10.1016/j.mrrev.2014.04.002

[262] Takagi, T., Moribe, H., Kondoh, H. and Higashi, Y. (1998) DeltaEF1, a Zinc Finger and Homeodomain Transcription Factor, Is Required for Skeleton Patterning in Multiple Lineages. Development, 125, 21-31.

[263] Gregory, P.A., Bert, A.G., Peterson, E.L., Barry, S.C., Tsykin, A., Farshid, G., Vadas, M.A., Kew-Goodall, Y. and Goodall, G.J. (2008) The miR-200 Family and miR-205 Regulate Epithelial to Mesenchymal Transition by Targeting ZEB1 and SIP1. Nature Cell Biology, 10, 593-601. https://doi.org/10.1038/ncb1722

[264] Niu, K., Shen, W.J., Zhang, Y.H., Zhao, Y. and Lu, Y.X. (2015) miR-205 Promotes Motility of Ovarian Cancer Cells via Targeting ZEB1. Genes, 574, 330-336. https://doi.org/10.1016/j.gene.2015.08.017

[265] Song, S.Z., Yu, W.H., Lin, S., Zhang, M.B., Wang, T., Guo, S. and Wang, H.B. (2018) LncRNA ADPGK-AS1 Promotes Pancreatic Cancer Progression through Activating ZEB1-Mediated Epithelial-Mesenchymal Transition. Cancer Biology \& Therapy, 19, 573-583. https://doi.org/10.1080/15384047.2018.1423912

[266] Sonohara, F., Yamada, S., Takeda, S., Hayashi, M., Suenaga, M., Sunagawa, Y., Tashiro, M., Takami, H., Kanda, M., Tanaka, C., Kobayashi, D., Nakayama, G., Koike, M., Fujiwara, M. and Kodara, Y. (2020) Exploration of Exosomal Micro RNA Biomarkers Related to Epithelial-to-Mesenchymal Transition in Pancreatic Cancer. Anticancer Research, 40, 1843-1853. https://doi.org/10.21873/anticanres.14138

[267] Xie, F., Huang, Q., Wang, C., Chen, S., Liu, C., Lin, X., Lv, X. and Wang, C. (2020) Downregulation of Long Oncoding RNA SNHG14 Suppresses Cell Proliferation and Invasion by Regulating EZH2 in Pancreatic Ductal Adenocarcinoma (PDAC). Cancer Biomarkers, 27, 357-364. https://doi.org/10.3233/CBM-190908

[268] Heurtier, V., Owens, N., Gonzalez, I., Mueller, F., Proux, C., Mornico, D., Clerc, P., Dubois, A. and Navarro, P. (2019) The Molecular Logic of Nanog-Induced Self-Renewal in Mouse Embryonic Stem Cells. Nature Communications, 10, 1109. https://doi.org/10.1038/s41467-019-09041-Z 
[269] Veneti, Z., Gkouskou, K.K. and Eliopoulos, A.G. (2017) Polycomb Repressor Complex 2 in Genomic Instability and Cancer. International Journal of Molecular Sciences, 18, Article 1657, 1-16. https://doi.org/10.3390/ijms18081657

[270] Han, T., Jiao, F., Hu, H., Yuan, C.C., Wang, L., Jin, Z.L., Song, W.F. and Wang, L.W. (2016) EZH2 Promotes Cell Migration and Invasion but Not Alters Cell Proliferation by Suppressing E-Cadherin, Partly through Association with MALAT-1 in Pancreatic Cancer. Oncotarget, 7, 11194-11207.

https://doi.org/10.18632/oncotarget.7156

[271] Li, S., Li, Y., Chen, B., Zhao, J., Yu, S., Tang, Y., Zheng, Q., Li, Y., Wang, P., He, X. and Huang, S. (2018) exoRBase: A Database of circRNA, lncRNA and mRNA in Human Blood Exosomes. Nucleic Acids Research, 46, D106-D112. https://doi.org/10.1093/nar/gkx891

[272] Wang, Y.H., Ji, J., Wang, B.C., Chen, H., Yang, Z.H., Wang, K., Luo, C.L., Zhang, W.W., Wang, F.B. and Zhang, X.L. (2018) Tumor-Derived Exosomal Long Noncoding RNAs as Promising Diagnostic Biomarkers for Prostate Cancer. Cell Physiol Biochem, 46, 532-545. https://doi.org/10.1159/000488620

[273] Lau, C., Kim, Y., Chia, D., Spielmann, N., Eibl, G., Elashoff, D., Wei, F., Lin, Y.L., Moro, A., Grogan, T., Chiang, S., Feinstein, E., Schafer, C., Farrell, J. and Wong, D.T.W. (2013) Role of Pancreatic Cancer-Derived Exosomes in Salivary Biomarker. The Journal of Biological Chemistry, 288, 26888-26897. https://doi.org/10.1074/jbc.M113.452458

[274] Cheng, L., Sun, X., Scicluna, B.J., Coleman, B.M., Andrew, F. and Hill, A.F. (2014) Characterization and Deep Sequencing Analysis of Exosomal and Non-Exosomal miRNA in Human Urine. Kidney International, 86, 433-444. https://doi.org/10.1038/ki.2013.502

[275] Sagredo, A.I., Santiago, A., Sepulveda, S.A., Roa, J.C. and Oróstica, L. (2017) Exosomes in Bile as Potential Pancreatobiliary Tumor Biomarkers. Translational Cancer Research, 6, S1371-S1383. https://doi.org/10.21037/tcr.2017.10.37 


\section{Abbreviations}

\section{Section (1) - Section (5)}

$\mathrm{PDAC}=$ pancreatic ductal adenocarcinoma

KRAS $=$ Kirsten rat sarcoma viral oncogene homolog

$\mathrm{CDKN} 2 \mathrm{~A}=$ cyclin-dependent kinase inhibitor $2 \mathrm{~A}$, or p16

TP53 = tumor protein 53; in fact, the encoded protein p53 is a strong tumor suppressor

SMAD4 $=$ mothers against decapentaplegic homlog-4

RTKs $=$ receptor tyrosine kinases

PI3K = Phosphatidylinositol-3 kinase

$\mathrm{PIP}_{2}\left(\mathrm{PI}(4,5) \mathrm{P}_{2}\right)=$ Phosphatidylinositol 4,5-bisphosphate

PDK1 = phosphoinositide-dependent protein kinase-1, or the master kinase

$\mathrm{ER}=$ endoplasmic reticulum

S6K1 = ribosomal protein kinase $\beta$-1 P70-S6K

Akt $(\mathrm{PKB})=$ protein kinase $\mathrm{B}(\mathrm{PKB})$, a serine-threonine protein kinase

mTOR1,2 = mammalian target of rapamycin complex 1,2

Rheb $=$ Ras homolog enriched in brain

S6K1 = ribosomal protein kinase $\beta$-1 P70-S6K

HSP $=$ heat shock protein; HSF $=$ heat shock factor

AMPK $=5$ ' adenosine monophosphate-activated protein kinase

Tuberous TSC1, 2 = scherosis complex (unit 1 or 2); TSC1 is also called harmartin and TSC2 is also named tuberin

FOXO $=$ Forkhead box protein (with members $1,2,3$ )

PTEN $=$ phosphatase and tensin homolog protein; phosphatase and tensin homologue deleted on chromosome-10

$\mathrm{LKB} 1=$ liver kinase $\mathrm{B} 1$ protein

VEGF $=$ vascular endothelial growth factor

$4 \mathrm{E}-\mathrm{BP} 1$ = eukaryotic translation initiation factor $4 \mathrm{E}$ (eIF4E)-binding protein 1 , encoded by the gene EIF4EBP1

HIF- $1 \alpha$ = hypoxia-inducible factor- $1 \alpha$

eNOS $=$ endothelial nitric oxide synthase

$\mathrm{NO}=$ nitric oxide

FNDC5 $=$ fibronectin type III domain-containing protein 5

PPAR- $\gamma=$ peroxisome proliferator-activated receptor- $\gamma$

$\mathrm{UCP} 1=$ Uncoupling protein 1

KAHA = ketoacid-hydroxylamine

PLC-epsilon $1=$ pholipase enzyme, type epsilon 1

DAG $=$ diacylglycerol

Ras $=$ (encoded by the gene KRAS) which is a member of a class of proteins called small GTPase

Raf = Rapidly Accelerated Fibrosarcoma, a member of serine/threonine-specific protein kinases related to retroviral oncogene

Mek $=$ Mitogen-activated protein kinase 
Erk $=$ Extracellular signal-regulated kinase

RSK = Ribosomal s6 family kinases

PanIN = intraepithelial neoplasia

$\alpha$-SMA $=\alpha$-smooth muscle actin

Fak $=$ focal adhesion kinase

VASP $=$ Vasodilator-stimulated phosphoprotein

Rho $\mathrm{A}=\rho$ factor, type $\mathrm{A}$, is a prokaryotic protein

Rock $=$ Rho-associated protein kinase

MLCK = Myosin light-chain kinase

Piezo1,2 = Piezo-type mechanosensitive ion channel component 1,2

PSCs $=$ pancreatic stellate cells

VASP $=$ Vasodilator-stimulated phosphoprotein

$\mathrm{CDKN} 2 \mathrm{~A}=$ cyclin-dependent kinase inhibitor $2 \mathrm{~A}$

CDK2,4,6 = cyclin dependent kinase 2,4,6

$\mathrm{Hdm} 2=$ human double minute 2

p21 = cyclin-dependent kinase inhibitor 1

$\mathrm{pRB}=$ retinoblastoma protein, the key "check-point" protein for cell cycle

$\mathrm{E} 2 \mathrm{~F}=$ protein encoded one of the genes that codifies a family of transcription factors in higher eukaryotes. Three of them are activators: E2F1, 2, and E2F3a. Six others act as suppressors: E2F3b, E2F4-8

$\mathrm{DFO}=$ desferrioxamine

$311=2$-hydroxy-1-naphthylaldehyde isonicotinoyl hydrazone

NDRG1 $=\mathrm{N}$-myc downstream regulated gene 1

$\mathrm{Dp} 44 \mathrm{mT}=$ di-2-pyridylketone 4,4-dimethyl-3-thiosemicarbazone

Section (6) - Section (10)

YAP1 $=$ yes-associated protein 1 , also known as YAP or YAP65

$\mathrm{TAZ}=$ (transcriptional co-activator with a PDZ-binding domain; also known as WW domain containing transcription regulator 1, or WWTR1.The "classical formal name" is Tafezzin

TEAD protein $=$ transcriptional enhanced associate domain protein

VGLL4 $=$ Transcription cofactor vestigial-like protein 4

$\mathrm{Mdm} 2=\mathrm{a}$ member of the $\mathrm{E} 3$ Ubiquin ligase

LATS1 = Large tumor suppressor kinase 1 (an enzyme with four members in the family, labeled 1-4)

Ptpn14/PTPN14 = protein tyrosine phosphatase non-receptor type 14

PanIN = intraepithelial neoplasia

Furin $=$ a serine protease which can cleave a plethora of proteins at polybasic recognition motifs

Kibra $=$ kidney and brain expressed protein $($ KIBRA) or WW domain-containing protein 1 (WWC1)

$\mathrm{c}$-JNKs $=\mathrm{c}$-Jun N-terminal kinases

LIMID1 = LIM domain-containing protein 1

ROR $\alpha=$ orphan nuclear receptor $\alpha$ 
AICAR = 5-Aminoimidazole-4-carboxamide ribonucleotide

SMAD2,3,4 = mothers against decapentaplegic homlog-2,3,4

PAI-1 = Plasminogen activator inhibitor-1

Akap12 = protein A-kinase anchor protein 12

ITGB6 $=$ Integrin beta- 6 , the $\beta 6$ subunit of the integrin $\alpha v \beta 6$

LAP $=$ Latency-associated peptide

ALDH1,2,3 = aldehyde dehydrogenase type 1,2,3

BMP-4 = bone morphogenetic protein, type 4

GLIS3 = Zn-finger transcription factor GLI-similar-3

$\mathrm{EGF}=$ epidermal growth factor

Jak = Janus kinase, with four members: Jak1, Jak2, Jak3, Tyk2

Stat protein $=$ signal transducer and activator of transcription protein

$\mathrm{SOC}=$ suppressor of cytokine

SNARE $=$ soluble $\mathrm{N}$-ethylmaleimide-sensitive fusion attachment protein receptor and syntaxin 6 is a member of this family

COPI = coat protein complex I", vesicle

$\mathrm{ARF}=\mathrm{GTPase}$ ADP-ribosylation factor

NF- $\kappa \mathrm{B}=$ Nuclear Factor-kappa B

MCP-1 $(\mathrm{CCL} 2)=$ chemokines monocyte chemoattractant protein 1

CXCL1 = chemokine C-X-C motif-ligand 1

Lymphotoxin $=$ a member of the Tumor Necrosis Factor (TNF) family of cytokines

$\mathrm{IKK}=\mathrm{I} \kappa \mathrm{B}$ kinase, consists of two members $(\mathrm{IKK} \alpha$ and $\mathrm{IKK} \beta)$

$\mathrm{NEMO} / \mathrm{IKK} \gamma=$ a regulatory subunit named NF- $\mathrm{KB}$ essential modulator

IKK $\gamma=$ inhibitor of nuclear factor kappa-B kinase subunit $\gamma$

$\mathrm{NIK}=\mathrm{NF}-\kappa \mathrm{B}$-inducing kinase, whose activity triggers off the non-canonical pathway.

$\mathrm{LT} \beta \mathrm{R}=$ Lymphotoxin $\beta$ receptor

TNF $\alpha=$ Tumour Necrosis Factor $\alpha$, an inflammatory cytokine produced

by macrophages/monocytes

COX-2 = cyclooxygenase- 2

MCP-1 = chemokines monocyte chemoattractant protein 1

CXCL1 = chemokine (C-X-C motif) ligand 1, recruiting immunity cells during angiogenesis and inflammation

Icam $1=($ Inter $)$ cellular adhesion protein 1

Vcam 1 = vascular cell adhesion protein 1

iNOS = inducible nitric oxide synthase iNOS

Bcl-2 = B-cell lymphoma 2

iNOS = inducible nitric oxide synthase

Collagenase1 degrades collagens of certain types

$\mathrm{I} \kappa \mathrm{B} \alpha=$ inhibitory $\kappa$ protein type $\alpha$

NF $\kappa B 1,2=$ NF- $\kappa$ B transcription factor type1, 2 respectively

$\mathrm{NIK}=\mathrm{NF}-\kappa \mathrm{B}$-inducing kinase 
LT $-\beta R=$ Lymphotoxin receptor type $\beta$

RelA = v-rel reticuloendotheliosis viral oncogene homolog A (avian), is also called p65

RelB = v-rel reticuloendotheliosis viral oncogene homolog B (avian)

$\mathrm{Ca}=$ carbonic anhydrase, $\mathrm{Ca} 9$ is driven by HIF-1 activity

APE1/Ref-1 = Apurinic/Apyrimidinic Endonuclease-1-Reduction/oxidation Effector Factor 1

APACHE II = Acute Physiologic Assessment and Chronic Health Evaluation II

Patch 1,2 $=$ Protein patched homolog 1,2

$\mathrm{Smo}=$ Smoothened protein in the hedgehog pathway

Sufu $=$ The suppressor of fused homolog, involved in the hedgehog pathway

Gli1 = protein containing five successive repeats of a zinc finger which can bind to DNA for transcription

Shh $=$ Sonic hedgehog

Ihh = Indian hedgehog

Dhh $=$ Desert hedgehog

Myc $=$ member of the family first established after the discovery of homologybetween an oncogene carried by the Avian virus, MyelocytomatosisBcl-2 = B-cell lymphoma 2, a regulator of apoptosis

Sox 2 = sex-determining region Y-box 2, a transcription factor that is essential for maintaining self-renewal stem cells

IPMN = intraductal papillary mucinous neoplasm

$\mathrm{M}-\mathrm{CPA} / \mathrm{PTX}=\mathrm{a}$ polymeric micelle formulation containing cyclopamine (CPA), and a cytotoxic chemotherapy drug paclitaxel (PTX)

\section{Section (11) - Section (15)}

ADAM10 = Disintegrin And Metalloproteinase Domain-Containing Protein 10

Nicd = intracellular domain of the Notch receptor

CSL $(\mathrm{CBF}-1)=$ DNA binding protein or transcriptional regulator, alias Suppressor of Hairless, Lag-2

MAML1 = mastermind-like protein 1

Hes1 = hairy and enhancer of split-1, a transcription factor

Hey1 = Hairy/enhancer-of-split related with YRPW motif protein 1

$\operatorname{COX} 2=$ Cyclooxygenase 2 , is an enzyme responsible for the synthesis of prostaglandins

MMPs $=$ The enzymes matrix metalloproteinases

ESA $=$ epithelial surface antigen

CTGF $=$ Connective tissue growth factor

PDGF $=$ Platelet-derived growth factor

EGCG $=$ green tea polyphenol epigallocatechin gallate

PDX1 = pancreatic and duodenal homeobox 1

ZEB1 = Zinc Finger E-Box Binding Homeobox 1, a transcriptional repressor 
that has been identified as an inducer of EMT

ALDH1 = aldehyde dehydrogenase, member 1

PACs = pancreatic acinar cells

PDCs $=$ pancreatic ductal cells

PanIN = intraepithelial neoplasia state

PDX1 = pancreatic and duodenal homeobox 1

shRNA = short hairpin RNA, an artificial RNA molecule with a tight hairpin turn that can be used to silence target gene expression via RNA interference

Snail $=$ a member of a family of transcription factors that promote the inhibition of the adhesion molecule E-cadherin to regulate epithelial to mesenchymal transition (EMT)

Section (16) - Section (21)

ATRA = All-trans-retinoic acid

TRF $=$ tocotrienol rich fraction

RHAMM = Hyaluronan mediated motility receptor (motility)

$\mathrm{VDR}=\mathrm{Vitamin} \mathrm{D}$ receptor

PEGPH20 = Pegvorhyaluronidase alfa

miRNA $=$ microRNA

ELF3 = transcription factor E74-like factor 3

GAPDH = glyceraldehyde-3-phosphate dehydrogenase

$\mathrm{SP}=$ small population

HPDE = human pancreatic ductal epithelial cells

MTA-1,2 = metastasis-associated protein MTA-1,2

BITC $=$ Benzyl isothiocyanate

$\mathrm{EVs}=$ extracellular vesicles

ncRNA = noncoding RNA

circRNA = circular RNA

piRNAs $=$ piwi-interacting RNAs

ceRNA = competing endogenous RNA

lncRNA = long non-coding RNA

IPMN = intraductal papillary mucinous neoplasms

$\mathrm{MCN}=$ mucinous cystic neoplasms

ZEB1,2 = Zinc finger E-box binding homeobox 1,2

$\mathrm{EZH} 2=$ Enhancer of zeste homolog 2

PRC2 $=$ Polycomb repressive complex 2 